Florida International University FIU Digital Commons

$7-7-2010$

\title{
Blood-Brain Barrier in vitro Model: A Tissue Engineering Approach and Validation
}

Zhiqi Zhang

Florida International University, zzhan001@fiu.edu

DOI: $10.25148 /$ etd.FI10081003

Follow this and additional works at: https://digitalcommons.fiu.edu/etd

\section{Recommended Citation}

Zhang, Zhiqi, "Blood-Brain Barrier in vitro Model: A Tissue Engineering Approach and Validation" (2010). FIU Electronic Theses and Dissertations. 246.

https://digitalcommons.fiu.edu/etd/246 


\section{FLORIDA INTERNATIONAL UNIVERSITY}

Miami, Florida

BLOOD-BRAIN BARRIER IN VITRO MODEL: A TISSUE ENGINEERING

APPROACH AND VALIDATION

A dissertation submitted in partial fulfillment of the

requirements for the degree of

DOCTOR OF PHILOSOPHY

in

BIOMEDICAL ENGINEERING

by

Zhiqi Zhang

2010 
To: Dean Amir Mirmiran

College of Engineering and Computing

This dissertation, written by Zhiqi Zhang, and entitled Blood-Brain Barrier in vitro Model: A Tissue Engineering Approach and Validation, having been approved in respect to style and intellectual content, is referred to you for judgment.

We have read this dissertation and recommend that it be approved.

$\begin{array}{r}\hline \text { Ophelia Weeks } \\ \hline \text { Nikolaos Tsoukias } \\ \hline \text { Wei-Chiang Lin } \\ \hline \text { Yen-Chih Huang } \\ \hline \text { Chenzhong Li, Major Professor }\end{array}$

Date of Defense: July 7, 2010

This dissertation of Zhiqi Zhang is approved.

Dean Amir Mirmiran College of Engineering and Computing

Interim Dean Kevin O'Shea University Graduate School

Florida International University, 2010 


\section{DEDICATION}

I dedicate this dissertation to my parents and all the animals that have to be sacrificed for scientific researches. 


\section{ACKNOWLEDGMENTS}

I wish to thank the members of my committee for their patience and support. Special thanks to Dr. Chenzhong Li - if it weren't because of him, I won't be able to accomplish my dissertation. And I'm always grateful for Dr. Eric Crumpler - if it weren't because of him, this dissertation won't even exist. 


\section{ABSTRACT OF THE DISSERTATION \\ BLOOD-BRAIN BARRIER IN VITRO MODEL: A TISSUE ENGINEERING \\ APPROACH AND VALIDATION}

by

Zhiqi Zhang

Florida International University, 2010

Miami, Florida

\section{Professor Chenzhong Li, Major Professor}

This dissertation evaluated the feasibility of using commercially available immortalized cell lines in building a tissue engineered in vitro blood-brain barrier (BBB) co-culture model for preliminary drug development studies. Mouse endothelial cell line and rat astrocyte cell lines purchased from American Type Culture Collections (ATCC) were the building blocks of the co-culture model. An astrocyte derived acellular extracellular matrix (aECM) was introduced in the co-culture model to provide a novel in vitro biomimetic basement membrane for the endothelial cells to form endothelial tight junctions. Trans-endothelial electrical resistance (TEER) and solute mass transport studies were engaged to quantitatively evaluate the tight junction formation on the invitro $\mathrm{BBB}$ models. Immuno-fluorescence microscopy and Western Blot analysis were used to qualitatively verify the in vitro expression of occludin, one of the earliest discovered tight junction proteins. Experimental data from a total of 12 experiments conclusively showed that the novel $\mathrm{BBB}$ in vitro co-culture model with the astrocyte derived aECM (CO+aECM) was promising in terms of establishing tight junction formation represented by TEER values, transport profiles and tight junction protein 
expression when compared with traditional co-culture (CO) model setups and endothelial cells cultured alone. Experimental data were also found to be comparable with several existing in vitro $\mathrm{BBB}$ models built from various methods. In vitro colorimetric sulforhodamine B (SRB) assay revealed that the co-cultured samples with aECM resulted in less cell loss on the basal sides of the insert membranes than that from traditional coculture samples. The novel tissue engineering approach using immortalized cell lines with the addition of aECM was proven to be a relevant alternative to the traditional $\mathrm{BBB}$ in vitro modeling. 


\section{TABLE OF CONTENTS}

CHAPTER

PAGE

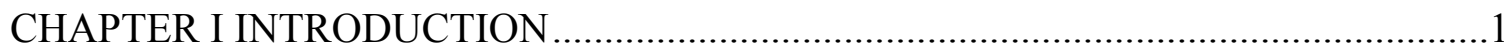

1.1 General Statement of Research Problem Area ......................................................... 1

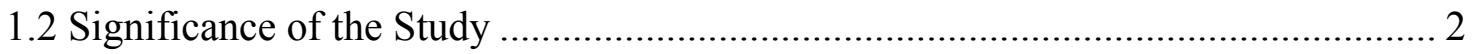

1.3. Research Premise and Methodology .............................................................. 3

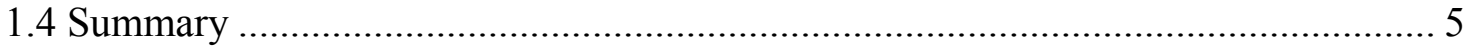

CHAPTER II OVERVIEW OF BLOOD-BRAIN BARRIER ..........................................

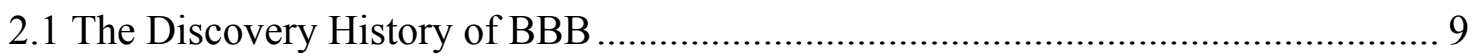

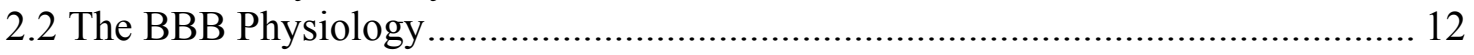

2.2.1 The Gate-Keeping Barrier ……………………....................................... 12

2.2.2 BBB Anatomical Features ....................................................................... 15

2.2.2.1 Brain Capillary Endothelial Tight Junctions......................................... 15

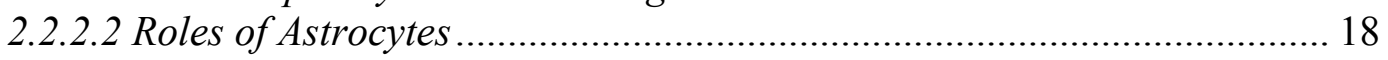

2.2.2.3 Contribution of Pericytes ........................................................................ 20

2.3 Current CNS Drug Delivery Examples .............................................................. 21

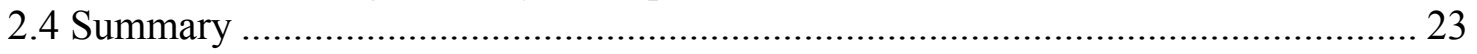

CHAPTER III ASTROCYTES AND EXTRACELLULAR MATRIX............................24

3.1 Astrocytes' Development and Morphologies....................................................... 24

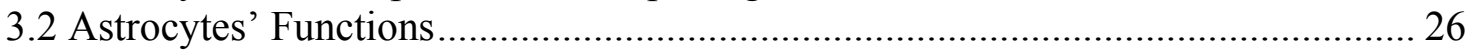

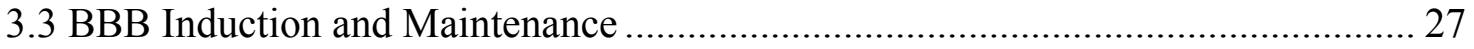

3.4 Secretion of Extracellular Components.............................................................. 28

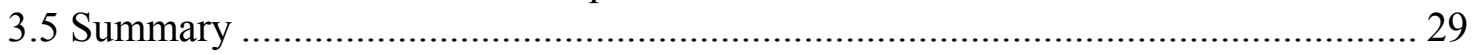

4.1 Noticeable Marks during Tissue Culture Development .......................................... 31

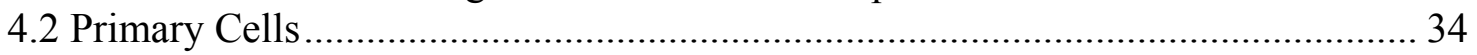

4.3 Immortalized Cell Lines................................................................................... 36

4.4 Choice between Primary and Immortalized Cell Lines.......................................... 37

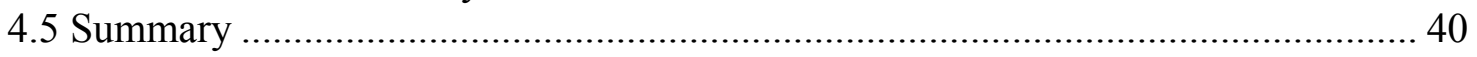

CHAPTER V ENGINEERING IN VITRO BBB MODEL ..............................................41

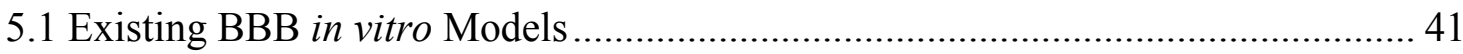

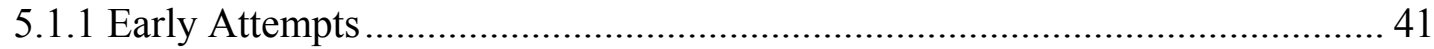

5.1.2 Current Attempts ......................................................................................... 41

5.1.2.1 Primary Bovine Brain Endothelial Cells (BBEC) .................................. 42

5.1.2.2 Primary Human Brain Endothelial Cells (HPBEC) ................................ 42

5.1.2.3 Immortalized Rat Brain Endothelial Cells (SV-ARBEC) .......................... 42

5.1.2. 4 Immortalized Mouse Brain Endothelial Cells (MBEC4) .......................... 43

5.1.2.5 Madin-Darby Canine Kidney Cell Lines(MDCK) ................................... 43

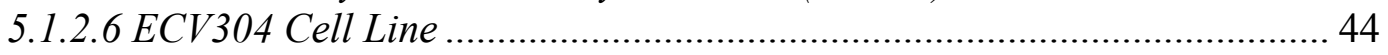

5.1.2.7 Human Colon Adenoma Derived Cell Line (Caco-2)................................ 44

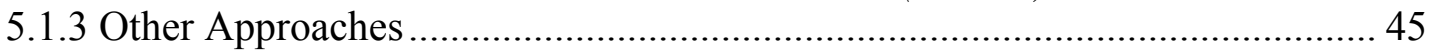

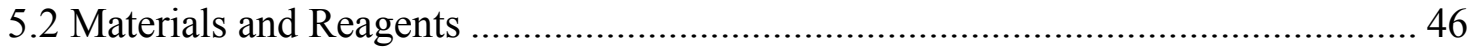




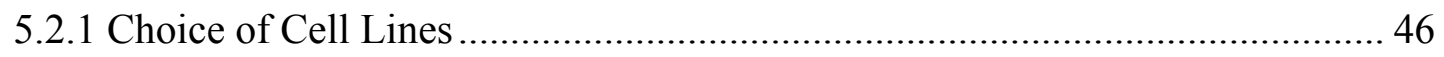

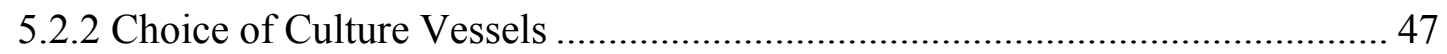

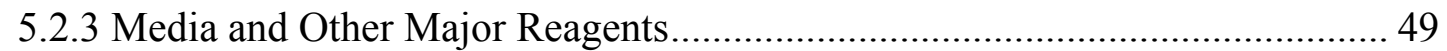

5.2.4 Other Equipments and Lab Wares.............................................................. 51

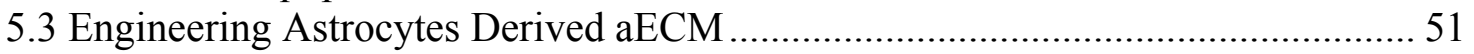

5.3.1 Theoretical Basis .................................................................................. 51

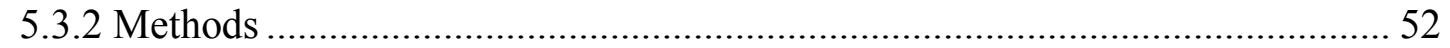

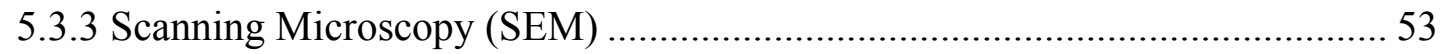

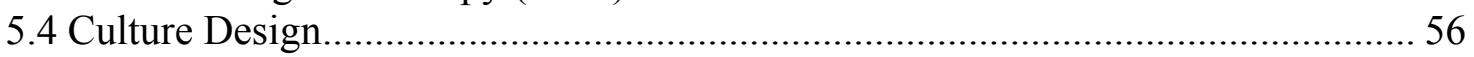

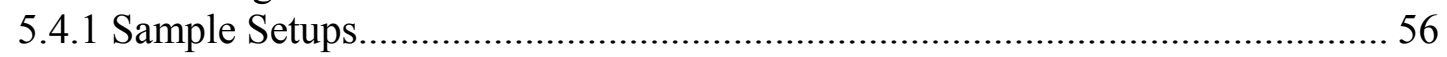

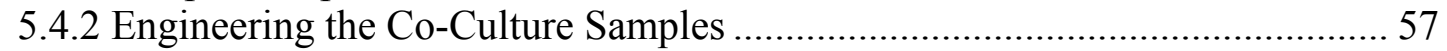

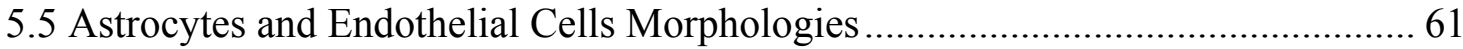

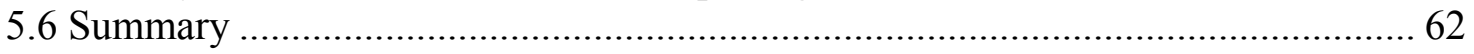

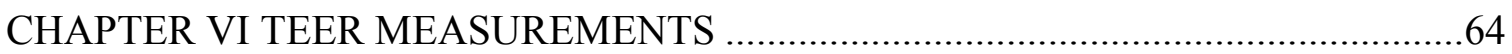

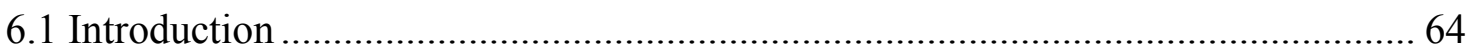

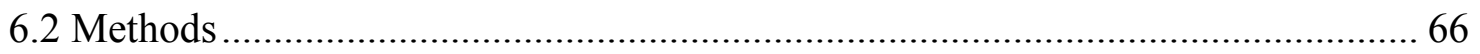

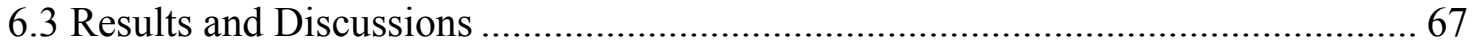

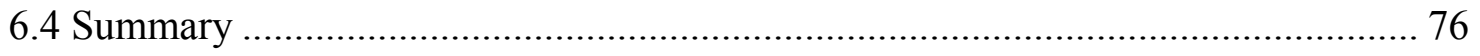

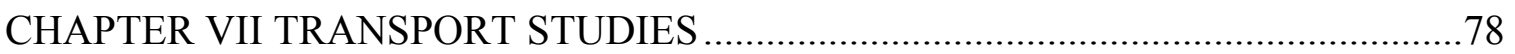

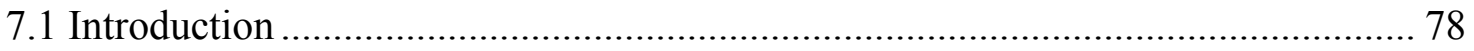

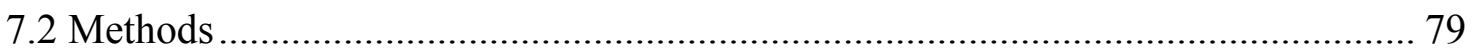

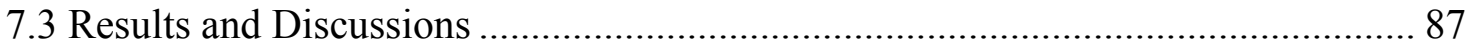

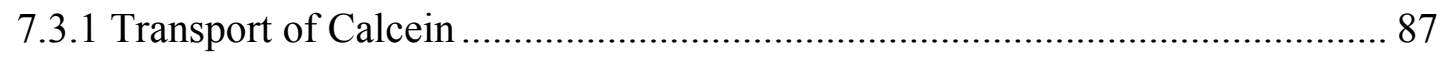

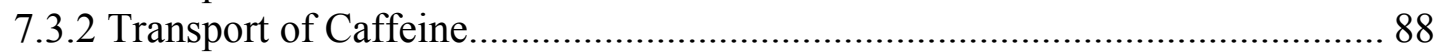

7.3.3 Transport of DOX

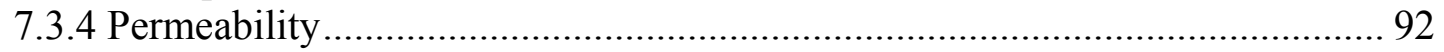

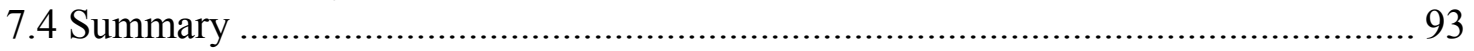

CHAPTER VIII TIGHT JUNCTION PROTEIN EXPRESSION ....................................95

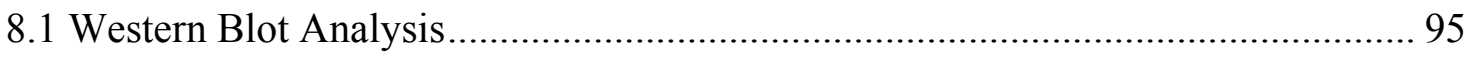

8.1.1 Introduction to Western Blot Techniques....................................................... 95

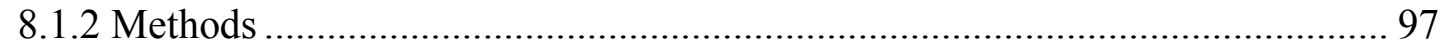

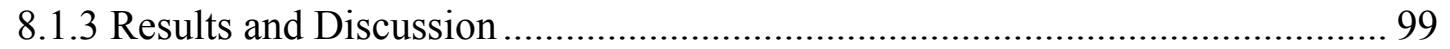

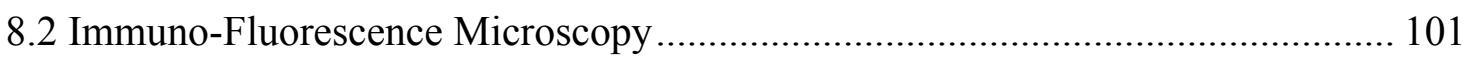

8.2.1 Introduction to Immuno-Fluorescence Microscopy ........................................ 101

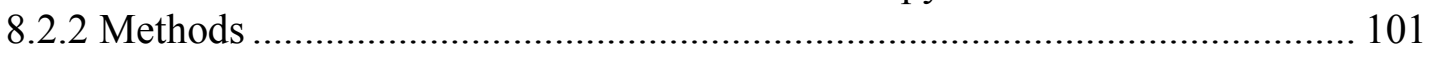

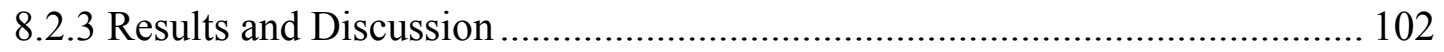

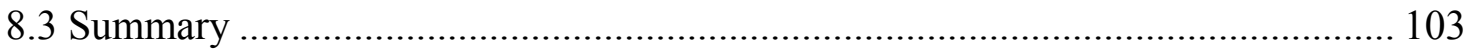

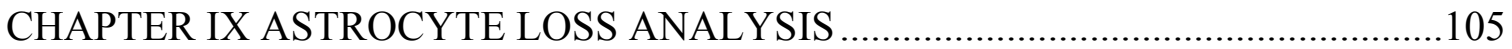

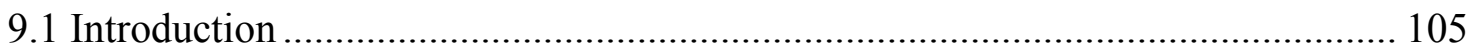

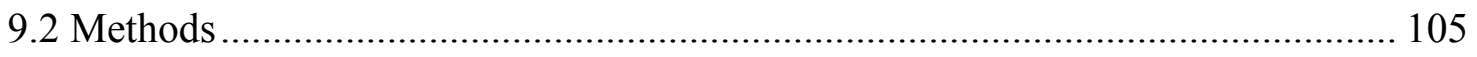

9.3 Astrocytes Cell Number SRB Calibration Curve................................................. 106

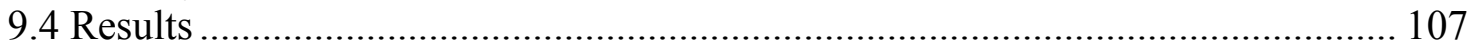




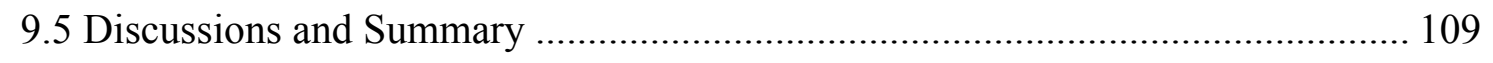

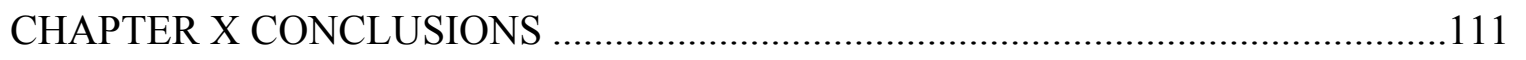

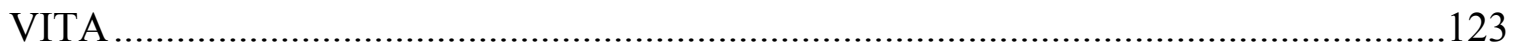




\section{LIST OF TABLES}

TABLE

PAGE

Table 1 Comparison between vasculatures between BBB and peripheral tissue 17

Table 2 Noticeable events/discoveries in the development history of the tissue and

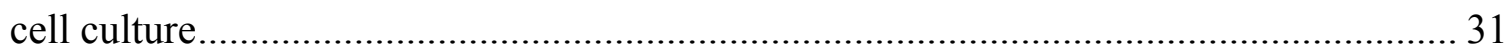

Table 3 Specifications of Corning Costar ${ }^{\circledR} \operatorname{Transwell}^{\mathrm{R}}$ inserts for $24-w e l l$ plates ........... 48

Table 4 Proposed test compounds in the BBB transport studies ................................. 78

Table 5 Serial dilution concentration demonstration............................................. 82

Table 6 Permeability of different test compounds across different sample setups.......... 93

Table 7 Cells fell onto well bottoms from different co-culture setups in 4 trials .......... 108 


\section{LIST OF FIGURES}

FIGURE

PAGE

Figure 1 The schematic drawings of blood-brain barrier and blood-cerebral spinal fluid barrier 11

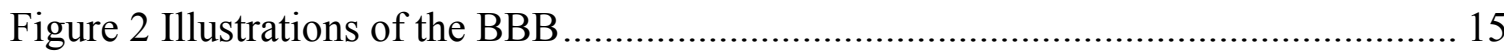

Figure 3 Tight junction's molecular components ................................................... 16

Figure 4 The schematic drawing of interactions between astrocyte endfeet and brain endothelial cells 19

Figure 5 Morphology of an astrocyte, which shows a typical star-like morphology ....... 25

Figure 6 Morphology of astrocyte in the BBB, with certain polarity ............................. 26

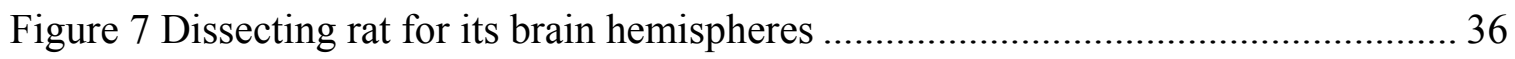

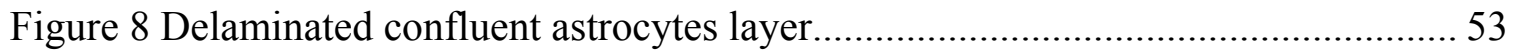

Figure 9 SEM picture of confluent astrocyte layer and decellularized astrocytes layer

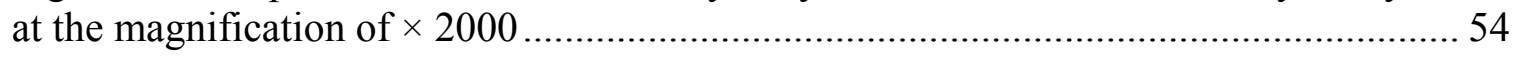

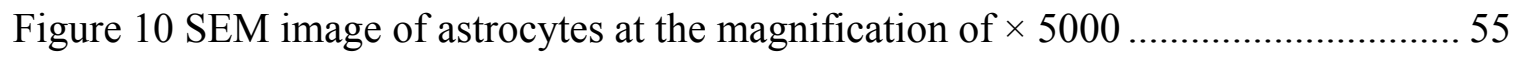

Figure 11 Demonstration of astrocyte single cell in SEM, commercially available

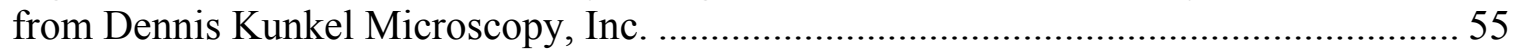

Figure 12 Schematic demonstrations of selected sample setups in the study .................56

Figure 13 Schematic drawing of steps in building "CO" and "CO+aECM" samples ...... 57

Figure 14 Astrocytes seeded on inverted inserts in the deep dish. A: side view;

B: top view

Figure 15 Sample plates. A: demonstration of how the inserts situated in the well plates. B: one batch of samples 60

Figure 16 Astrocytes seeded on the inverted inserts in the incubator...

Figure 17 Cell morphologies observed from optical microscope under magnification of $\times 200$.

Figure 18 TEER measurement instrument. A: EVOM Endothelial Voltohmmeter.

B: STX2 electrodes measuring TEER value of the insert in the well plate. 
Figure 19 Endohm TEER measurement chamber for $6.5 \mathrm{~mm}$ diameter Transwell inserts .

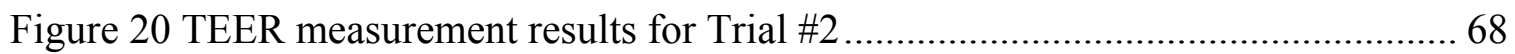

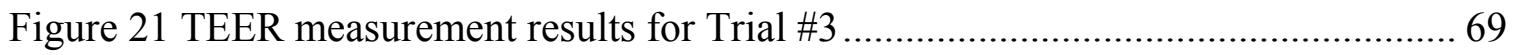

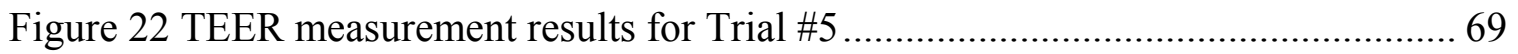

Figure 23 TEER measurement results for Trial \#6 ................................................ 70

Figure 24 TEER measurement results for Trial \#7 f.............................................. 70

Figure 25 TEER measurement results for Trial \#8 ..................................................... 71

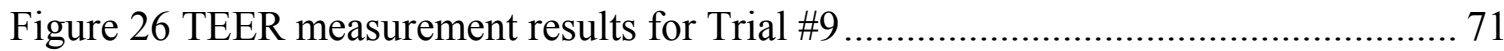

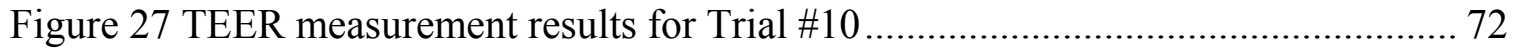

Figure 28 TEER measurement results for Trial \#11 f................................................. 72

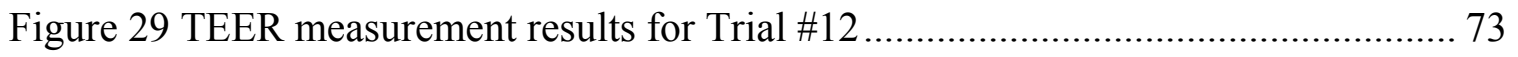

Figure 30 TEER measurement results for Trial $\# 13$.................................................. 73

Figure 31 Measurement instruments in the transport studies. A: Cary $100 \mathrm{UV}-\mathrm{Vis}$ spectrophotometer. B: Silica spectrophotometer cuvette.......................................... 80

Figure 32 Example of calcein concentration serial dilution scans............................... 84

Figure 33 Example of calcein concentration serial dilution Absorbance vs.

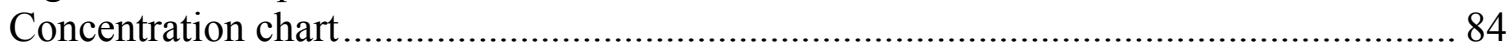

Figure 34 Example of caffeine concentration serial dilution Absorbance vs.

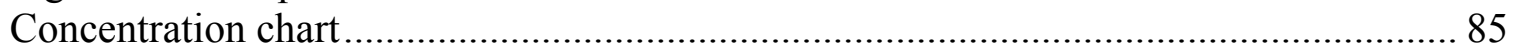

Figure 35 Example of DOX concentration serial dilution Absorbance vs.

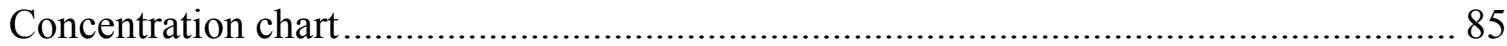

Figure 36 Example of sucrose concentration serial dilution Absorbance vs.

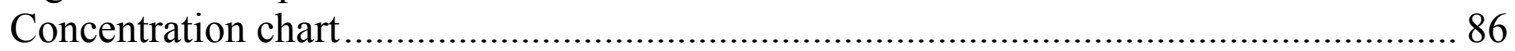

Figure 37 Calcein mass transport profiles. ............................................................. 90

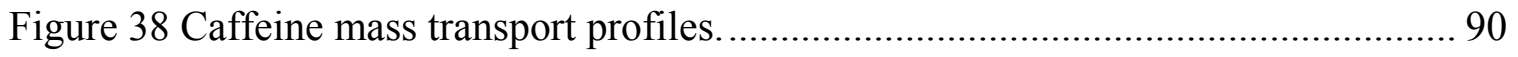

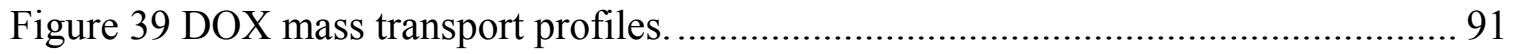


Figure 40 Sandwich assembly for Western Blot gel transfer .................................... 98

Figure 41 Scan of exposed film from the Western Blot analysis of occludin ............... 100

Figure 42 Immuno-fluorescence microscopy image of endothelial layers from co-culture samples: (A): CO sample; (B): CO+aECM sample.................................... 103

Figure 43 Dried SRB stained 24-well plate for the calibration curve.......................... 107

Figure 44 Astrocytes SRB Calibration Curve ..................................................... 108

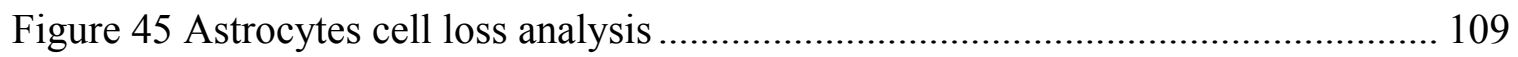




\section{LIST OF ABBREVIATIONS}

aECM: acellular extracellular matrix;

AS: one of the culture setups where the astrocytes were cultured alone;

ATCC: American Type Culture Collection;

BBB: blood-brain barrier;

CNS: central nervous system;

CO: one of the culture setups where endothelial cells were cultured on one side of the insert membrane while astrocytes were cultured on the basal side of the inserts;

$\mathrm{CO}+\mathrm{aECM}$ : one of the culture setups where endothelial cells were seeded onto a piece of aECM on the apical side of the insert while astrocytes were cultured on basal side of the inserts;

COL inserts: Corning Costar ${ }^{\circledR}$ Collagen I \& III coated Transwell ${ }^{\mathrm{R}}$ inserts for 24 -well plates;

CSF: cerebrospinal fluid;

DOX: Doxorubicin, a type of chemotherapy agent;

EC: one of the culture setup where endothelial cells were cultured alone;

aECM on the apical side of the insert;

ECM: extracellular matrix;

HRP: horseradish peroxidase;

MW: molecular weight;

TEER: trans-endothelial electrical resistance;

WHO: World Health Organization. 


\section{CHAPTER I INTRODUCTION}

\subsection{General Statement of Research Problem Area}

According to World Health Organization (WHO), brain diseases such as cerebrovascular disease and Alzheimer's disease, as well as epilepsy and brain tumors have become a significant healthcare problem in western countries. According to the Brain Tumor Society, over 200,000 people are diagnosed with a primary or metastatic brain tumor in the US each year. The drug development for the central nervous system (CNS) will have to grow over five times to be comparable to those of the cardiovascular diseases(1). The biggest challenge in brain drug delivery research is to overcome the brain's effective protective mechanism collectively known as the blood-brain barrier (BBB) (2-5). With the existence of the (BBB), which greatly restricts the influx of almost everything into the brain, the treatment methods are largely limited on the drug delivery aspect. Current available drug delivery methods are usually risky, highly invasive, can lead to irreversible side effects or allow very limited distribution of drug within the brain tissue -- for example, injecting hyper osmotic sugar solutions (Mannitol) into a brain artery, inserting catheters to pump drugs into the brain and implanting drug eluting wafers after surgical tumor removal(6). The limited treatment methods have also disappointed the hope of cure of patients. It is necessary and important to create a feasible and repeatable research model for the blood-brain barrier along the journey of conquering brain diseases.

Despite numerous models established by different research labs on different cell types, it is still difficult to conclude a specific model to be the optimal prototype. The in vitro models do not always show promising consistency and are reported to poorly 
correlate with the in vivo studies(7). In this particular study, in vitro BBB co-culture model will be established utilizing commercially available cell lines. Astrocytes derived acellular extracellular matrix (aECM) will be introduced as a biomimetic basement membrane of the endothelial cells for better seeding and induction of brain microvasculature endothelial cells features. Essential characteristics of the in vitro coculture model will be evaluated to validate the feasibility of the study. Study will be focused on morphological study of endothelial monolayer, trans-endothelial electrical resistance (TEER) value to evaluate the tightness of intercellular junctions, expression of characteristic tight junction protein such as Occludin using Western Blot and immuno fluorescent microscopy as well as transport studies for various compounds across the in vitro model membrane, and etc.

\subsection{Significance of the Study}

According to the complexity in patients and the problem of cost efficiency, it is always better to perform in vitro studies before going into in vivo studies. With the brain disease being major killer and the blood-brain barrier remaining the major obstacle for medicine treatment, a valid in vitro model will help greatly in understanding the disease as well as drug development. The use of BBB in vitro culture assays in early safety assessment of drugs is a growing field (5). There are many existing so-called BBB in vitro models but none of them are perfect. The aim of my study is to provide a cost effective yet relatively easy to obtained source of BBB in vitro model for preliminary drug studies. Commercially available immortalized cell lines will be employed as the building blocks of the in vitro co-culture model, this approach will save significant amount of work in the initial preparation of cells in comparison with primary cells 
obtained directly from live animals. At the same time, in the current society where animal rights is more and more an important issue, the method utilizing passages of immortalized cell lines is consistent with people's value which could lead to prolonged development of the current research. In addition, the introduction of aECM in engineering the in vitro co-culture model is a novelty in the BBB in vitro modeling. This approach enables better mimic of the brain environment with the aECM serving as the basement membrane for the endothelial cells. The importance of extracellular matrix (ECM) has been now more and more widely accepted. This particular approach is a practical utilization of the ECM in the real experiment and has shown promising results. This practice will aid better understanding and wider utilization of ECM in the scientific studies.

\subsection{Research Premise and Methodology}

The aim of this study is to provide researchers with a reliable and reproducible in vitro blood-brain barrier model for preliminary drug delivery studies. Two types of cell lines will the employed in engineering an in vitro co-culture model. The co-culture model will be built on a cell culture insert cultured in cell culture well plates. One type of cell will be seeded on the top of insert membrane while the other type of cell seeded on the other side of the insert membrane. The two types of cell lines are immortalized endothelial cells and astrocytes purchased from American Type Culture Collection (ATCC). Astrocytes derived aECM is engineered in the lab and employed in the coculture for improved in vitro BBB characteristics. Multiple combinations of cell culture setups, such as endothelial cells cultured alone (EC), astrocytes cultured alone (AS), co-

culture without aECM $(\mathrm{CO})$ and co-culture with $\mathrm{aECM}(\mathrm{CO}+\mathrm{aECM})$ will be tested for 
the decision of an optimal model. While the $\mathrm{CO}+\mathrm{aECM}$ sample is the proposed ultimate goal, other setups as well as blank inserts are used as side-by-side controls.

All the sample setups are subjected to several different tests to evaluate the formation of tight junction as the most important characteristic of the BBB. First of all, TEER measurements are performed daily starting the second day after the endothelial cells on the top of insert membranes are seeded. This test serves as a screening method in the initial evaluations for all the different sample setups. Setups with consistent low TEER values will be excluded from further studies as BBB is featured on high TEER values. After the initial TEER value screening, the remaining sample setups will be evaluated by transport study testing several different compounds such as calcein, caffeine and Doxorubicin (DOX). The transport profiles are recorded as one of but not solely the main reference to evaluate the BBB formation. Qualitative assays such Western Blot and immuno-fluorescent microscopy will also be employed in the assessment to identify the existence of Occludin, one of the earliest discovered tight junction proteins. Western blot compares the expression level of Occludin in the cell lysates collected at the end of culture period from different setups, while immuno-fluorescent microscopy enables one to see the existence of Occludin directly on the sample membranes. At last, a brief SRB assay will be utilized to quantify the amount of astrocytes fall off onto the bottom of the well plates over the culture period for the co-culture samples. This approach is employed to monitor the integrity of the in vitro models besides checking the tight junction formation only. 


\subsection{Summary}

This study is about utilizing commercialized immortalized cell lines to build twocell-type co-culture in vitro $\mathrm{BBB}$ model, with the induction of aECM to induce better endothelial brain like characteristics and functionality. There are ten chapters in this dissertation, each with its specific focus.

Chapter II is the overview of the BBB. The chapter starts with the introductions of the discovery history of BBB. How the barrier was initially discovered can be dated back to more than one hundred years ago. Various studies by many different scientists have been carried out in finding and confirming the existence of the BBB featuring brain endothelial tight junction. How to distinguish between blood-brain barrier and bloodcerebral spinal fluid barrier is also described by schematic drawings. Followed by the discovery history of the $\mathrm{BBB}$, the most accepted $\mathrm{BBB}$ characteristics are introduced in this chapter. Several key features and functionalities are discussed in depth with graph demonstrations and list of tables. Anatomical physiology of the BBB is introduced later in this chapter. The three-cell model including brain microvasculature endothelial cells, astrocytes and pericytes is the current widely accepted BBB model. The functions and contributions of each type of cell are introduced briefly in this chapter. In the end of the chapter, several current drug delivery methods to the CNS are briefly discussed by mechanisms, methods, applications, advantages and disadvantages.

Chapter III emphasizes on the contribution of astrocytes as one of the most important building blocks of the $\mathrm{BBB}$, and its role in the extracellular matrix is also explored. A detailed description of how the acellular extracellular matrix derived from astrocytes is introduced in this chapter too. 
Chapter IV starts with an elegant summary of the milestones in the history of tissue and cell culture presented by MCBI's knowledge base. With such necessary background knowledge, the chapter then continues with the introduction of primary cells and immortalized cell lines, respectively. Then the comparison of the advantages and disadvantages between primary cell lines and immortalized cell lines are made, with several issues being extensively discussed. The conclusions of why the author chose the immortalized cell lines are made in the end of the chapter.

Chapter V focuses on the engineering of the in vitro BBB model. The chapter begins with the introduction of several different BBB models attempts made in the earlier ages, recent years and some unpopular ways. Each attempt is introduced by the type of cells, setup method, evaluation methods and conclusions with the available information. Discussions and comparisons of the advantages and disadvantages are briefly made. Then as the major part of this chapter, detailed procedures of how the BBB in vitro co-culture model is engineered are described. How to engineer astrocytes derived aECM is introduced first. Scanning microscopy pictures of a confluent layer of astrocytes and decellularized aECM are shown to distinguish these two very different layers. Optical microscopy morphology pictures of cultured cells on the inserts as well as on the well bottoms are shown. Each different sample setup is described and explained as well as the engineering of the astrocytes derived aECM. A schematic drawing summarizing all the different sample setups helps to better understand each model sample.

Chapter VI compares TEER values of different samples setups including "EC," "CO," "CO+aECM" and some other setups for a total of 12 trials. The setups with 
consistent low TEER values will be excluded from further study since high TEER value is one of the most important features of the BBB.

Chapter VII tests solute transport of several different compounds across the sample membranes. The testing compounds include calcein, caffeine and DOX. The measurement method is different from the most popular current radio-labeling methods because of the limitations of the available facility on campus. The testing compounds were dissolved by serum free media to reach suitable concentration readable for a spectrophotometer in the UV-visible range. Thus optical absorbance was read by the spectrophotometer, subsequently solute concentration and mass transport profiles are calculated using optical absorbance vs. concentration calibration curve. Effective permeability coefficients are calculated and transport profiles are compared and discussed between different compounds as well as between different sample setups.

Chapter VIII examined the expression of Occludin, one of the earliest found tight junction proteins using Western Blot and immuno-fluorescent microscopy. The western blot focused on examining occludin expression level in astrocytes alone, EC alone, $\mathrm{CO}$ and $\mathrm{CO}+\mathrm{aECM}$ sample lysates, comparing with occludin positive sample. Immunofluorescent images are taken under a fluorescent microscope for co-culture samples treated with antibodies that recognize occludin and conjugated with fluorescent dye. Occludin contours and the density of tight junctions are compared and discussed in the specific study. The study in the chapter further demonstrated CO+aECM samples is a better setup in helping the endothelial cells better exhibit BBB like features such as tight junction protein expression. 
Chapter IX shows a minor study examining the astrocytes cell loss from the basal sides of the inserts from the co-culture samples by the SRB assay. The cell numbers were determined by converting the optical absorbance value obtained from the micro plate reader in the experiments using an experimentally obtained cell number calibration cruve. Results showed that astrocytes cell loss from co-culture with aECM samples were much less than those from the co-culture samples alone. The integrity of the BBB is partly reflected in part by this study.

Chapter $\mathrm{X}$ is the conclusion chapter. The assumptions are testified throughout the studies and the co-cultured $\mathrm{BBB}$ in vitro model with $\mathrm{aECM}$ is the optimal setup among all the sample setups. Astrocytes derived aECM plays important role in the in vitro BBB formation and the immortalized cell lines are approved to be practical in building the $\mathrm{BBB}$ in vitro model. 


\section{CHAPTER II OVERVIEW OF BLOOD-BRAIN BARRIER}

\subsection{The Discovery History of BBB}

The brain functions within a well-controlled environment separate from the milieu of the periphery. The mechanisms that control the unique environment of the brain are collectively referred to as the "blood-brain barrier."

It has been over a century that the concept of $\mathrm{BBB}$ has been recognized for its physiological existence. In the late $19^{\text {th }}$ century, Paul Ehrlich, the German immunologist, performed an experiment where water soluble dyes were intravenously administered(8). As the result, it was found the body tissue as well as the choroid plexus were stained, but the brain was failed to be stained, and the cerebrospinal fluid (CSF) had no color. It was first thought the dyes did not have binding affinity to stain the brain. But later in the beginning of the $20^{\text {th }}$ century, Paul's student, Edwin Goldman proved for the same dye to stain the brain tissue but was not able to pass a certain barrier to reach into the brain. On the other hand, another experiment where dyes were injected into the brain through subarachnoid space showed instead the CSF was stained but not the peripheral tissues. This also proved the existence of the 'barrier.' But, it was not until 1950 that Tschirgi showed the main reason for the series dye experiments were the dyes bound to albumin, the plasma protein, which was widely used in present studies as the agent to test the in vitro $\mathrm{BBB}$ models.

It was in 1898 the blood-brain barrier was confirmed at the cerebral vessels level by Bield and Kraus(9). They observed sodium ferro-cyanide or cholic acids had no effects on the central nervous system (CNS) pharmacologically when injected intravenously. But when the same agent was injected intraventricularly, the neurological 
symptoms occurred after the injection. In 1890, Lewandowsky was the first one to introduce the term "blood-brain barrier" into the world.

Another series experiments were carried out by Goldman in 1909 and 1913 with the Trypan blue injection in animals to further prove the existence of blood brain barrier. The experiments also showed it was not because of the previous Ehrlich's dyes' binding affinity that the brains were not stained. Trypan blue is a dye first synthesized by Ehrlich. In the experiments, Trypan blue was injected to rabbits and dogs either through brain ventricular system or intravenously. Likewise, the brain tissue was stained when Trypan blue was injected through brain ventricular system, but, was colorless when the vital dye was injected intravenously while the whole animal turned blue as well as the choroid plexuses. Thus in 1913, Goldman introduced the hypothesis that the CSF was the vehicle responsible for transporting substances. This theory was also recognized as "way of the spinal fluid."

Later in 1942, Friedemann found that lipid soluble dye was able to travel across the cerebral microvasculature and stained the brain. Broman in 1941 introduced his findings of two barrier systems in the brain: on at the choroid plexus called bloodcerebrospinal fluid and the other at the cerebral microvasculature called blood-brain barrier(10). Figure 1 shows the schematic drawings of these two barriers. The blood-brain barrier features continuous layer of endothelial cells connected by tight junctions as the lining of capillaries, while the blood-cerebrospinal fluid barrier (BCSFB) is composed of choroid plexuses and the arachnoid membranes of the circumventricular organs. The barrier function of BCSFB is provided by the junctions between epithelial cells in the choroid plexus which are slightly more permeable than those of the BBB. Broman later 
argued that the capillary endothelial cells while not the astrocytic endfeet should be responsible for the blood-brain barrier function.

\section{A. Blood-Brain Barrier}

Brain Capillary

a) Endothelial Cell

Blood

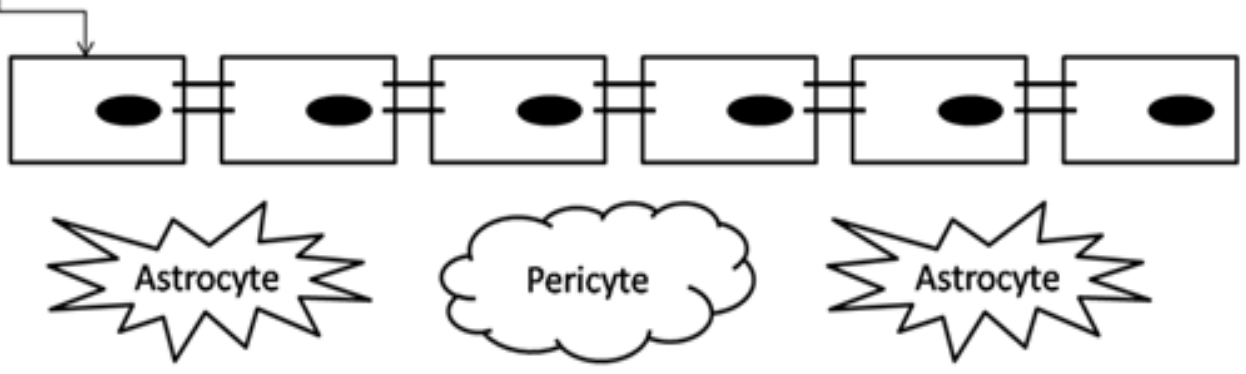

Brain Extracellular Fluid

\section{B. Blood-Cerebral Spinal Fluid Barrier}

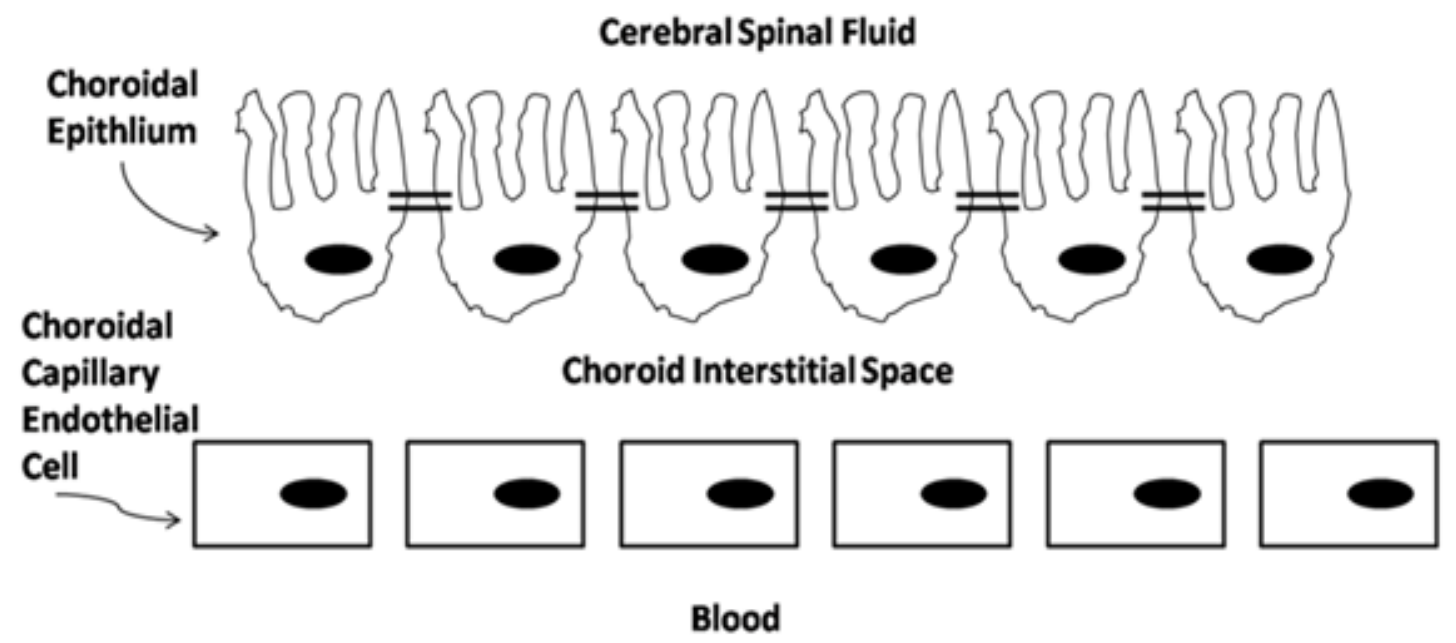

Figure 1 The schematic drawings of blood-brain barrier and blood-cerebral spinal fluid barrier

Thanks to the development of high resolution electron microscopy, as well as the sensitive tracer methods; the structural basis for the BBB was not cleared to the astrocytic 
and endothelial level until 1967. It was revealed by Reese and Karnovsky ultrastructurally using electron-sense tracer horseradish peroxidase (HRP) showed that endothelial cells in mouse cerebral capillaries formed a structural barrier to HRP, thus the statement of BBB is "an endothelial barrier present in capillaries that course through the brain" was made(11). Here HRP is a glycoprotein with a molecular weight of 39,800 . It can be cytochemically determined at both light and electron microscopic level. Reese and Karnovsky found the HRP could travel through the first luminal inter-endothelial tight junctions. The tight junctions are referred to as the junctions between the adjacent endothelial cells in the blood-brain barrier, where the morphology appeared to be continuous; pinocytotic vesicles could hardly be found and not engaged in the transport. Other experiments with smaller protein tracers such as microperoxidase and ion lanthanum were tested by intravenous injection by Feder in 1971 and Brightman and Reese in 1969, respectively. All these experiments proved the tight junctions in BBB's ability to actively keep out specific substances from entering the brain interstitial fluid.

There have been extensive studies and controversial conclusions about the development and maturation of $\mathrm{BBB}$ in vertebrates, from embryo to several days after birth, and the data may vary between species. Since the study in this research will be focusing on matured blood-brain barrier, the development or the maturation of the BBB will not be further discussed in this chapter.

\subsection{The BBB Physiology}

\subsubsection{The Gate-Keeping Barrier}

William Pardridge has done extensive research in the BBB modeling area and in his review in 2003; eloquent study has been carried out on the BBB's impact in the CNS 
drug delivery(12). The BBB virtually restricts all molecules except the small and lipophilic ones from entering the cerebral space. This is an important mechanism to protect the brain by avoid the variation in plasma constitution. For instance, the internal environment will not fluctuate much after meal, exercising and will not be disturbed too much when the body intakes some certain neurotransmitters or xenobiotics(13). On the other hand, to maintain an effective functional brain, some hydrophilic molecules such as oxygen and carbon dioxide still can go through the barrier and enter the brain by active transport where energy is required.

Only small lipophilic molecules with the molecular weight of 400-500 dalton (some say 600) or under can across the BBB freely(14). But, the problem is that only a few diseases, including epilepsy, depression and chronic pain, can benefit from the aforementioned route utilizing small molecules $(15,16)$. Alzheimer disease, brain cancer, stroke, HIV infection of the brain and many other brain related diseases are not treatable by those small molecular weight medicines. According to Pardridge, no pharmaceutical company has a drug-targeting program for the $\mathrm{BBB}(12)$. And there are certain limitations that small molecular weight drugs cannot achieve in the CNS drug delivery. First of all, there are only a few CNS diseases that response consistently to the lipophilic small molecular weight drugs as aforementioned. And even for the drugs that are able to permeate the $\mathrm{BBB}$, the active percentages of these drugs are reported to be extremely low in the CNS(17). Secondly, not all the small molecular weight drugs can effectively cross the $\mathrm{BBB}$ to reach a pharmacological significant amount. Molecules that have too many hydrogen-bond-forming groups, such as histamine, will have much difficult in crossing the $\mathrm{BBB}$ because of its rate-limiting role(14). 
With the help of transport proteins, essential nutrients for the brain such as glucose and some amino acids or related molecules are able to get into the brain by the relatively high concentrations of those proteins in brain endothelial cells. Also, some receptor mediated systems also exist on the BBB making it possible for some macromolecules to enter the brain. Transferrin receptor, GLUT-1 glucose carrier and L1 amino acid transporter are among the best known ones $(18,19)$. However, large molecular weight drugs for the CNS are not successfully developed because of the BBB. Even with the existence of various transporting systems on the BBB, no such 'transporter' has been found to be capable of effectively transporting larger molecules with small-molecule peptidomimetic functioning as a endogenous ligand to the transporters, let alone all the other larger molecular weight drugs, who are usual abandoned in the initial CNS drug development studies.

P-glycoprotein is another transporter found on luminal endothelial membrane as well as at astrocyte processes in the brains of both human and primate brains (20-22). But, this membrane protein works on the opposite direction to the other membrane proteins mentioned above. It basically expels a variety of substances out from the cerebral space that penetrated into the brain. P-glycoprotein is an essential component of the blood-brain barrier to maintain the homeostasis in the brain by keeping the potentially toxic substances from accumulating in the brain, interfering brain's normal function. Pglycoprotein is also the major protein expressed when evaluating the in vitro models of blood-brain barrier(23). 


\subsubsection{BBB Anatomical Features}

As a complex environment, the BBB is composed of brain capillary endothelial cells, astrocytes and pericytes $(7,24)$. Currently, the well-accepted blood-brain barrier is the aforementioned three-cell model: brain micro vascular endothelial cells lining in the cerebral space, together with the endfeet of astrocytes and pericytes to compose the complete blood-brain barrier(25). Figure 2 gives a schematic view for both the BBB three-cell model (A), as well as a traverse view showing how the brain capillary endothelial cells lining together forming tight junction and how they interact with the supporting astrocytes (B).
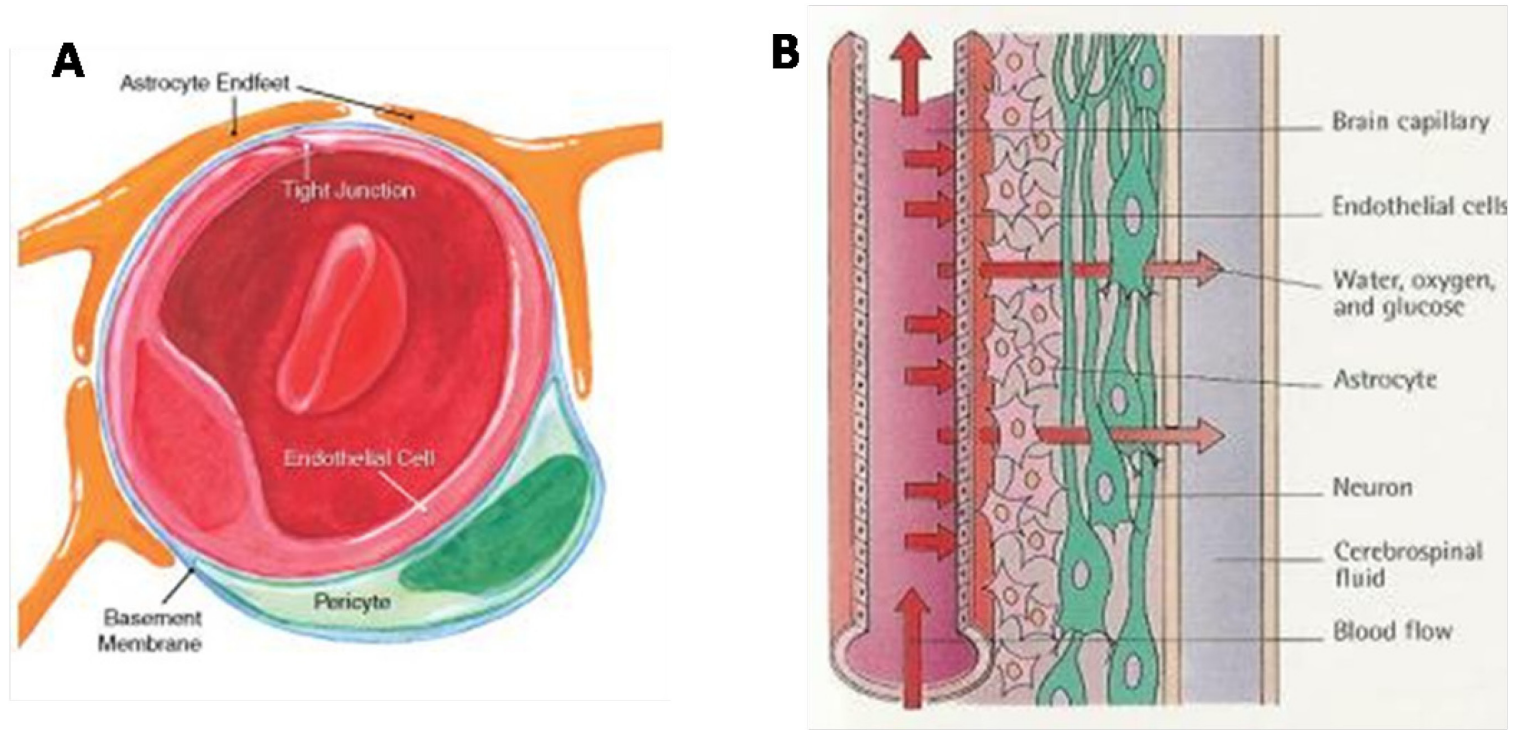

Figure 2 Illustrations of the BBB

\subsubsection{Brain Capillary Endothelial Tight Junctions}

The tight junctions in the $\mathrm{BBB}$ are characterized as the apical junctional complex between brain microvascular endothelial cells(26). The junctions between the endothelial cells in the brain capillaries are so tight that it is said to be on the $50 \sim 100$ folds level than peripheral vessels(27). The tight junctions greatly restrict the paracellular transport of 
water soluble substances so the transport is then mainly depended on transcellular pathways as described in the previous section. Solute transport study of several different molecules of various characteristics will be carried out for the in vitro model samples and the results will be discussed in detailed in later chapters.

One of the best ways to reflect the tightness of the junctions is by measuring the electrical resistance. Transendothelial electrical resistance (TEER) is the most widely used parameter to describe the resistance in the barrier to validate the in vitro models. TEER values measured in vivo can be as high as $2000 \mathrm{~cm}^{2}$, while the values in peripheral are only less than $100 \Omega \mathrm{cm}^{2}$. The TEER value measurement will be performed to check all the in vitro samples as an indicator of the integrity of the samples. Detailed procedure will be showed later in the method part.

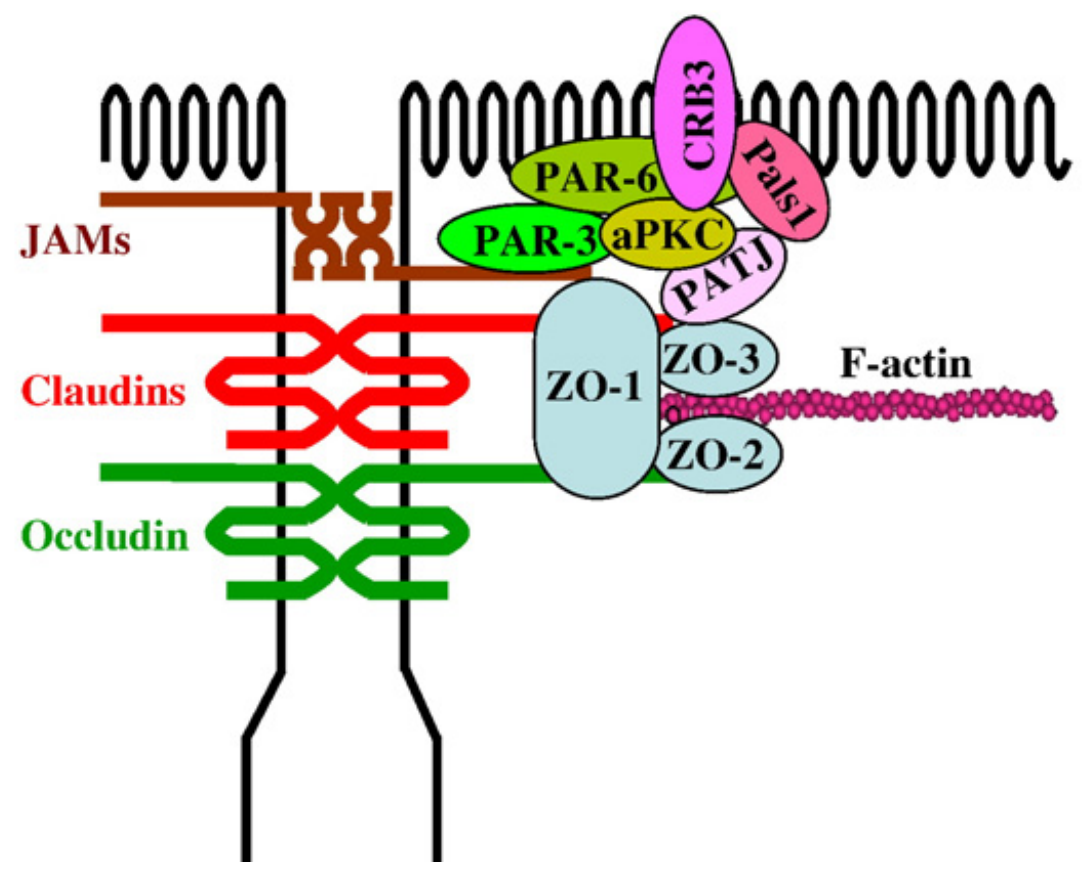

Figure 3 Tight junction's molecular components 
Table 1 Comparison between vasculatures between BBB and peripheral tissue

\begin{tabular}{|l|l|l|}
\hline Property & BBB Vasculature & Peripheral Vasculature \\
\hline Tight junctions & Present & Rare \\
\hline Pincocytosis & Rare & Abundant \\
\hline Fenestrae & Rare & Abundant \\
\hline Electric resistance & High $\left(\sim 2000 \Omega \mathrm{cm}^{2}\right)$ & Low $\left(<100 \Omega \mathrm{cm}^{2}\right)$ \\
\hline Perivascular space & Small & Extensive \\
\hline Alkaline phosphatase & Present & Absent \\
\hline$\gamma$-glutamyl transpeptidase & Present & Absent \\
\hline Monoamine oxidase & Present & Absent \\
\hline Glucose transporter & Present & Absent \\
\hline Cellular mitochondrial content & $\sim 10 \%$ & absent \\
\hline Receptor for transferrin \& insulin & Present & \\
\hline
\end{tabular}

Several molecular components are unique to the tight junction, such as occludin, claudins, ZO-1 and junctional adhesion molecules (JAMs) $(26,28)$. A schematic drawing of the BBB tight-junction components is shown in Figure 3. Schematic drawing is credited by Chiba et al.(34) Among these constituents of tight junction strands, occludin was the first to be identified(29). It is an approximately $60 \mathrm{kDa}$ transmembrane protein, whose C-terminal region binds to $\mathrm{ZO}-1$, which associates with actin cytoskeleton. Several studies showed that occludin may be involved in signal transduction as signal transmitter and thus an important component of the BBB (30-34). Western blot analysis 
and immuno-fluorescent study will be carried out to study the existence and expression level of occludin on the in vitro model samples.

The blood-brain barrier vasculature is unique and distinctive in comparison with the peripheral tissue vasculatures. Table 1 lists the comparison of some key features on the vasculatures between $\mathrm{BBB}$ and peripheral tissues. The existences of tight junction and membrane transporter proteins have been described in earlier sections. And because the active transport are taking places more frequently than the peripheral tissues, more cellular mitochondrial content is present to provide energy for such activities. High electric resistance is another important characteristic to distinguish $\mathrm{BBB}$ from the peripheral tissue vasculature, and this is also one of the numbers to evaluate the in vitro BBB co-culture model. The anatomical BBB physiology is next topic to cover.

\subsubsection{Roles of Astrocytes}

A series of studies have implied that it is not intrinsic for brain microvascular endothelial cells to demonstrate the BBB features. Rather, the CNS environment plays a critical role inducing the cells with the properties(36). Astrocytes are among the most recognized key components of the aforementioned environment. As early as in 1967, Davson and Oldendorf predicted astrocytes to be the major source responsible for inducing the BBB phenotype and specialization(37). Later studies proved the statement: cultured astrocytes were found to induce the tightening of endothelial cells when planted in the normal leaky sites(38). On the contrary, Stewart and Wiley found chick brain endothelial cells on mesenchymal tissue implants from quail developed into leaky capillaries(39). It was shown anatomically of the microvasculature in brain as in Figure 4

of how the astrocytes endfeet interact with endothelial cells(40). Rubin et al. suggested it 
is essential for the direct interaction between endothelial cells and astrocytes to build a most favorable $\mathrm{BBB}(41)$. It is also the basis for this particular dissertation research of astrocytes-endothelial cells co-culture systems.

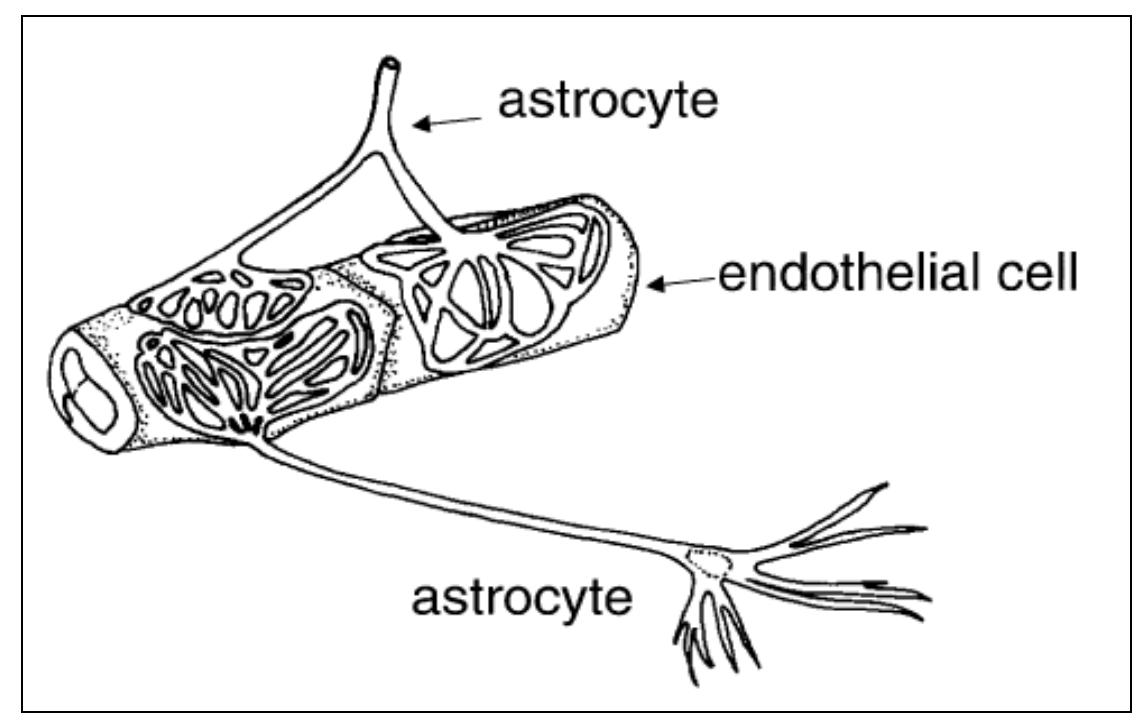

Figure 4 The schematic drawing of interactions between astrocyte endfeet and brain endothelial cells

Considerable efforts have been put into investigating the mediating agents to induce and maintain the BBB phenotype and its characteristics. $\gamma$-GTP (gamma-glutamyl transpeptidase) is a widely cited agent found on brain endothelium in situ responsible for amino acid transport. It was found great amount of $\gamma$-GTP was lost from primary brain endothelial cells shortly after they were isolated. The same thing happened to alkaline phosphatase (ALP), which is responsible for removal of phosphate groups. Glut-1, Asystem and the L-system carriers are reported to be up-regulate on the brain endothelial cells with the presence of astrocytic glial cells $(22,42)$. Transcytotic mechanisms for lowdensity lipoprotein (LDL) and expression of transferrin receptor are also reported to be up-regulated by the influence of the astrocytes presence(43). 
Some in vitro systems utilizing non-brain sourced endothelial cells such as HUVEC-Cs from human umbilical vein and endothelial cells from bovine aorta have shown BBB characteristics such as tight junctions or several specific markers(44). Even though there exist some controversial arguments questioning astrocytes signaling and regulating function, it is still widely testified and proved that astrocytes play important roles in inducing and maintaining the development of the blood-brain barrier phenotype. It is also considered the theoretical basis for this specific dissertation of the astrocytes-endothelial cells co-culture system. But it is also suggested brain microvascular endothelial cells are not the only cells responding to the signals of astrocytes.

\subsubsection{Contribution of Pericytes}

Pericytes are the cells shown in green in Figure 2 of the three-cell model. Clearly the pericyte is separated by the basement membrane from the EC and the endfeet of astrocytes. Morphologically, pericytes have round cell bodies, remarkable nucleus with plentiful lysosomes and abundant cytoplasmic processes $(45,46)$. The origin of the word 'pericyte' gives a straightforward hint of the cell, from the location point of view: 'peri-' means around and 'cyto-' means cell (47).

Pericytes are believed to offer structural stability on vessel wall and microvasculature vasodynamic capacities. It was observed on PDGF-B-deficient mice the development of microaneurysm with the deficit of pericytes(48). Several other facts also demonstrate the importance role of pericyte structurally maintaining the integrity of microvessels: diabetic patients with pericyte metabolic injury are reported to have retina microaneurysm formations(49); pericytes deterioration is found in cerebral hemorrhage with amyloidosis hereditarily. 
CNS pericytes are considered as 'housekeeping scavenger cells'(50-52). Some studies have tried to show pericyte's possible role in blood-brain barrier $(50,53,54)$. These studies provided evidence for the pericytes' involvement structural integrity, differentiation, angiogenesis regulation and genesis of blood-brain barrier. It was also suggested pericytes may take part in neuroimmune functions when they exhibit phagocytic activity.

Even though many mechanisms and implications of the CNS pericytes are still elusive, they have proved themselves to take part in virtually all processes on the bloodbrain barrier. There has not been successful in vitro cell culture models reported to the public. It will be very interesting to see a three-cell co-cultured in vitro model, which is also much more close to the in vivo situation, but then the cell culture work will become very complicated. It is still difficult to isolate CNS pericytes from primary culture, and definitive cell markers remains hard to identify. With better understanding and technical abilities, scientists will be able to investigate more on this important component of bloodbrain barrier.

\subsection{Current CNS Drug Delivery Examples}

The drug delivery routes to the brain are essentially to bypass the BBB. However, current available methods are usually risky, highly invasive, can lead to irreversible side effects or allow very limited distribution of drug within the brain tissue. Several most popular methods are briefly introduced as follows.

Direct administration of drugs into the brain includes intracerebral administration (ICA), intrathecal administration (ITA) and nasal administration (NA). Highly potent therapeutic agents with highly restricted area of distribution are always administered via 
ICA. The advantage of this method is that a wide range of compounds in spite of their sizes or formulations can be administered. However, this method is highly invasive, which requires a hole drilled in the head but still with very limited brain distribution for drugs of all sizes. ITA is often employed when local analgesia is needed. This method injects or infuses small lipophilic drugs into the CSF surrounding the spinal cord. CNS complications can almost always be avoided due to the rapid removal of these drugs from CSF to systemic bloodstream. However, proteins administered this way have much slower clearance rate from the CSF. Various compounds can be administered through olfactory neural pathway directly to CNS by NA. This method is rapid yet non-invasive, and does not need to modify the drugs or their carriers. Systemic side effects are also eliminated. However, since NA requires small administration volume, sufficient drug absorption amount is difficult to achieve.

BBB disruption can be achieved by modifications of tight junctions for temporarily controlled and increased permeability of the brain capillaries. Injecting hyper osmotic solutions such as mannitol can temporarily pull apart tight junctions due to shrinkage of cerebral endothelial cells and expansion of blood volume. Paracellular diffusion of hydrophilic compounds is enhanced by this method. One noticeable drawback is beta-galactosidase over expression due to this method.

BBB permeability can also be achieved in a concentration- and time-dependent disruption manner. Endogenous proinflammatory vasoactive agents such as histamine, bradykinin, and nitric oxide can all be employed by this method. However, the limitations are rather obvious: the method is very short-acting, has a narrow therapeutic window and also has significant dose-limiting side effects. Because of these limitations, analogs of 
these endogenous proinflammatory vasoactive agents are being studied and developed to facilitate better BBB disruption while reducing the side effects. However, there is still a long way to go on this route.

\subsection{Summary}

This chapter introduces the basic biology of the BBB, including the most popular three-cell model, and supporting introduction of the contributions of the three types of cells. The three types of cell are defined one by one in the order of endothelial cells, astrocytes and pericytes. Endothelial tight junction and tight junction proteins are introduced in the endothelial cells section. Astrocytes' role in inducing endothelial cells brain-like features are briefly mentioned in this chapter but will be explained in extended detail in the next chapter. The contributions of pericytes as the essential building block of BBB are also explained. Current CNS drug delivery methods have also been discussed in the end of the chapter. 


\section{CHAPTER III ASTROCYTES AND EXTRACELLULAR MATRIX}

\subsection{Astrocytes' Development and Morphologies}

Astrocyte is a type of glial cell which possess star-shaped morphology and glial fibrials as intermediate filaments. Copious extended astrocytic processes surround adjacent blood vessels and neural cells. However, it is not easy task to define astrocytes as will do for most other types of cells(55). The now widely-accepted concept is that astrocytes stand for a various population of cells with plentiful of functions. Characteristics of astrocytes are briefly summarized in following paragraphs for better understanding the definition of this special type of cell.

In mammals, astrocytes are found to be derived from various lineages, suggesting that astrocytes are created from different manners, which explains the diversity of astrocytes even in the same region of the brain(56, 57). For examples, grey matter astrocytes (protoplasmic astrocytes) are generated from embryonic radial glia, while white matter astrocytes (fibrous astrocytes) are generated from neonatal SVZ progenitors. Cai et al. performed a study on the astrocytes diversity in 2007 where they found out the grey matter astrocytes up-regulated GFAP in the absence of Olig2, while the formation of white matter astrocytes was greatly affected(58).

The morphology of astrocytes is heterogeneous and very complex. Astrocytes were originally named after their star-like shape morphology as depicted in Figure 5 demonstrated by Wang et al. (27). Astrocytes are recognized as "process-bearing cells" that exist throughout the nervous system but they do not possess dendrites or axons. However, astrocytes are heterogeneous as previously mentioned. For example, grey matter astrocytes have numerous branching processes, providing sheath to synapses and 
covering to the blood vessels with their endfeet. White matter astrocytes' processes are long, thin and unbranched. The endfeet of white matter astrocytes wrap nodes of Ranvier. The astrocytes that form BBB are known as "pituitary astrocytes pituicyte". These astrocytes' morphology presents certain polarity as Figure 6 shows.

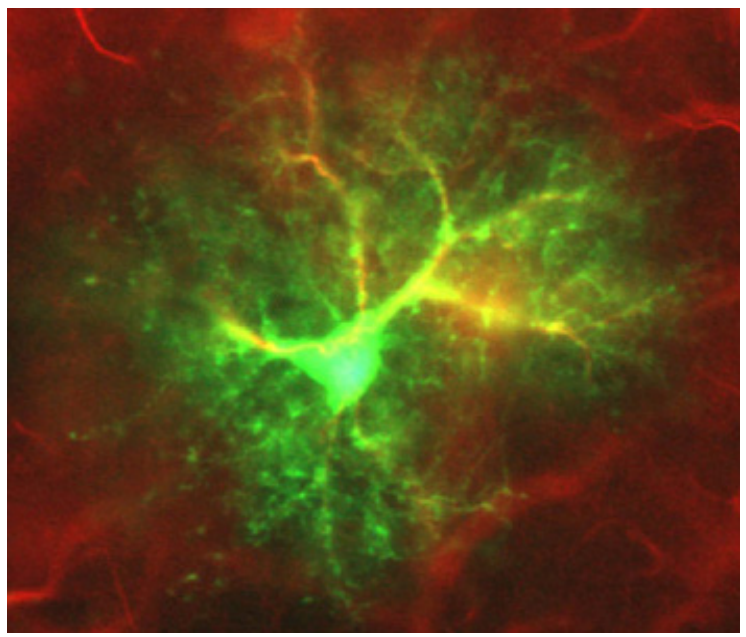

Figure 5 Morphology of an astrocyte, which shows a typical star-like morphology

Even though astrocytes are diversified based on their different antigenic and morphological features, they do share many general biophysical properties which led to the initial conclusion that astrocytes are "passive cells"(59). These biophysical properties are known by examples as: astrocytes' hyperpolarized resting membrane potentials (-80 or $-90 \mathrm{mV}$ ); under physiological conditions astrocytes do not generate action potentials; and large voltage-independent $\mathrm{K}^{+}$currents exist in astrocytes, which include cases when voltage-dependent $\mathrm{K}^{+}$currents are presented(60). However, the word "passive" is no longer being used with the advances of the astrocytes research. Controversies in the understanding of astrocytes still exist largely in the neural science research world and lots of further studies will have to be carried out to give us even better understanding of the astrocytes biophysical characteristics. 


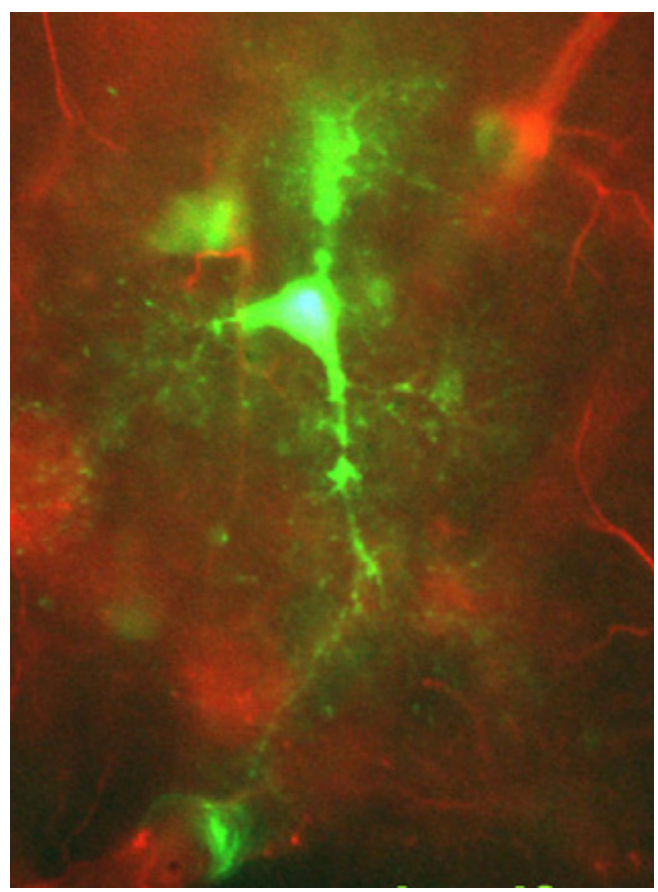

Figure 6 Morphology of astrocyte in the BBB, with certain polarity

\subsection{Astrocytes' Functions}

Astrocytes are known to express a large amount of receptors important in regulating self development as well as in responding to neurons and injuries $(61,62)$. The receptors expressed by astrocytes include ionotropic receptors, G-protein coupled receptors, receptors for chemokines, steroids, growth factors, and receptors related to innate immunity $(63,64)$.

One of the most noticeable astrocytes receptor expression characteristics is, cultured astrocytes are known to express nearly all the receptors exists in neurons as found when astrocytes are subject to acute slices or in vivo. This characteristic is very important especially in $\mathrm{BBB}$ co-culture modeling, making it possible for the in vitro $\mathrm{BBB}$ model to possess similar feature to mimic the in vivo situations. However, exceptions are not extinct for some cases, but the rare receptor express cases in astrocytes are not typical 
and of little importance in the BBB in vitro modeling assessment $(65,66)$. Astrocytes are also shown to adjust their receptor expression in accordance to their surrounding environment. Various receptors in different amount will be triggered to be expressed due to the change of environment such as in case of brain injury(67).

Well accepted astrocytes functions include BBB induction and maintenance, synthesizing adhesion molecules, extracellular matrix proteins, extracellular $\mathrm{K}^{+}$ buffering, metabolic support, angiogenesis, detoxification, immune functions, and many other functions. Actually, astrocytes are considered the major producer of adhesion molecules in the central nervous system (CNS). Evidence has shown that integrin matrix adhesion receptors secreted by brain astrocytes and endothelial cells are important in maintaining BBB permeability which will be discussed in more detail in next section(68, $69)$.

Because of the topic of this specific dissertation, astrocytes function in BBB induction and maintenance, and its function in extracellular matrix protein synthesis will be the discussion focus in following sections.

\subsection{BBB Induction and Maintenance}

As previously mentioned, brain capillary endothelial cells are enveloped by astrocytes endfeet - specialized processes sent by astrocytes, where they form rosette-like structures(40). Moreover, astrocytes are believed to induce and regulate the BBB, more specifically, the formation of endothelial tight junction and transport systems expression $(19,26)$.

Numerous reviews have shown that by co-culturing with astrocytes, tight junction proteins in brain endothelial cells isolated from the brain are up-regulated, thus able to 
maintain most of the BBB phenotype and characteristics(70, 71). Otherwise, if the isolated brain endothelial cells are cultured alone, certain BBB features will be lost(72). The aforementioned proteins being up-regulated in the existence of astrocytes are but not limited to GLUT1, P-glycoprotein, the L- and A- system amino acid carriers(42). These proteins are mentioned in previous chapters as important factors in the BBB characteristics.

Furthermore, studies found out that culture media taken from astrocytes overexpressing SSeCKS, known as conditioned media, helped to block angiogenesis both in vitro and in vivo. Such media also showed the ability to help increase tight junction proteins in cultured endothelial cells, thus reducing permeability to certain solute transport. Astrocytes are also believed to involve in the maintenance of BBB for its tight junction and microvascular permeability. Thus it is safe to conclude it will be beneficial to include astrocytes as a component of the in vitro $\mathrm{BBB}$ model to provide the endothelial cells brain like feature induction.

Last but not least, the BBB induction and maintenance is not a one-way street. Cultured astrocytes growth is also found to be reciprocally influenced by endothelial cells(73). Laminin production, glutamate synthetase activity and antioxidant enzyme activity are all proved to be regulated in such reciprocal way.

\subsection{Secretion of Extracellular Components}

In the CNS, astrocytes are the major cell in charge of secreting cell adhesion molecules (CAM) and extracellular matrix (ECM) proteins. Various studies have shown astrocytes' ability to regulate neurite growth by regulating the expression of certain molecules in response to in vitro environmental changes. Some of these molecules are 
Laminin, N-cadherin, fibronectin and neural CAM(74-76). Astrocytes are also reported to be involved in ECM degradation and remodeling by playing fundamental role by producing proteolytic enzymes, particularly matrix metalloproteinases(77-79).

Astrocytes are known to regulate neuronal maturation and survival by discharging growth factors such as fibroblast growth factor (FGF), brain-derived neurotrophic factor (BDNF), nerve growth factor (NGF), and etc(80). Cultured astrocytes are also capable of releasing growth factors in vitro. For example, neuronal survival and neuronal growth induction are enhanced by cilliary neurotrophic factor (CNTF) secreted by astrocytes, probably through a $\mathrm{Ca}^{2+}$ dependent pathway. Astrocytes can also release other molecules such as $\mathrm{S} 100 \mathrm{~B}$ to promote neurite outgrowth and astrocytic glutamate uptake in case of injury.

More recent researches keep finding out more and more astrocytes' involvement in ECM secretion such as its role to promote synaptogenesis between developmental CNS neurons in vivo and in vitro. The goal of research is to investigate the possibility to use astrocytes derived acellular-ECM (aECM) in the in vitro co-culture model building in order to help maintain the BBB properties of the co-cultured endothelial cells, and to increase the quality of the in vitro $\mathrm{BBB}$ system. The aECM is a piece of layers derived from confluent astrocytes in vitro culture where cells are lysed and washed away and only the astrocytes skeleton and the extracellular components are left for endothelial seeding. The procedures will be described in more detail in the methodology part.

\subsection{Summary}

This chapter focuses on introducing the astrocytes by study their development, morphology and functionalities. It is important to understand astrocytes' role in BBB 
induction and maintenance, which will be the theoretical support of building the in vitro model as a co-cultured system. Astrocytes' important role in ECM component secretion is also vital and gives the author inspiration of the novel approach where astrocytes derived aECM are introduced in engineering the co-culture BBB in vitro model. Move onto next chapter, the application of primary cells versus immortalized cell lines are going to be discussed and compared. 


\section{CHAPTER IV PRIMARY VERSUS IMMORTALIZED CELL LINES}

\subsection{Noticeable Marks during Tissue Culture Development}

Animal sourced cells have a long history of being used in scientific studies, being in vitro cultured since around 1900s. Cells for in vitro studies are majorly divided into two categories: primary cells that are derived directly from animal tissues, or immortalized cell lines specially treated that are able to be subcultured to an extensive passage number and utilized as cell banks(81). However, before introducing either of the cell lines, some noticeable events/discoveries in the development history of the tissue and cell culture should be acknowledged and appreciated in the following table summarized and presented by NCBI (Copyright (C) 2002, Bruce Alberts, Alexander Johnson, Julian Lewis, Martin Raff, Keith Roberts, and Peter Walter; Copyright (C) 1983, 1989, 1994, Bruce Alberts, Dennis Bray, Julian Lewis, Martin Raff, Keith Roberts, and James D. Watson.).

Table 2 Noticeable events/discoveries in the development history of the tissue and cell culture

\begin{tabular}{|l|l|}
\hline 1885 & $\begin{array}{l}\text { "Roux shows that embryonic chick cells can be maintained alive in a saline } \\
\text { solution outside the animal body." }\end{array}$ \\
\hline 1907 & "Harrison cultivates amphibian spinal cord in a lymph clot, thereby \\
demonstrating that axons are produced as extensions of single nerve cells."
\end{tabular}




\begin{tabular}{|c|c|}
\hline & ns." \\
\hline 1948 & $\begin{array}{l}\text { "Earle and colleagues isolate single cells of the L cell line and show that they } \\
\text { form clones of cells in tissue culture." }\end{array}$ \\
\hline 1952 & $\begin{array}{l}\text { "Gey and colleagues establish a continuous line of cells derived from a human } \\
\text { cervical carcinoma, which later become the well-known HeLa cell line." }\end{array}$ \\
\hline 1954 & $\begin{array}{l}\text { "Levi-Montalcini and associates show that nerve growth factor (NGF) stimulates } \\
\text { the growth of axons in tissue culture." }\end{array}$ \\
\hline 1955 & $\begin{array}{l}\text { "Eagle makes the first systematic investigation of the essential nutritional } \\
\text { requirements of cells in culture and finds that animal cells can propagate in a } \\
\text { defined mixture of small molecules supplemented with a small proportion of } \\
\text { serum proteins." }\end{array}$ \\
\hline 1956 & $\begin{array}{l}\text { "Puck and associates select mutants with altered growth requirements from } \\
\text { cultures of HeLa cells." }\end{array}$ \\
\hline 1958 & $\begin{array}{l}\text { "Temin and Rubin develop a quantitative assay for the infection of chick cells in } \\
\text { culture by purified Rous sarcoma virus. In the following decade the characteristics } \\
\text { of this and other types of viral transformation are established by Stoker, } \\
\text { Dulbecco, Green, and other virologists." }\end{array}$ \\
\hline 1961 & $\begin{array}{l}\text { "Hayflick and Moorhead show that human fibroblasts die after a finite number } \\
\text { of divisions in culture." }\end{array}$ \\
\hline 1964 & $\begin{array}{l}\text { "Littlefield introduces HAT medium for the selective growth of somatic cell } \\
\text { hybrids. Together with the technique of cell fusion, this makes somatic-cell } \\
\text { genetics accessible." }\end{array}$ \\
\hline
\end{tabular}




\begin{tabular}{|l|l|}
\hline 1965 & $\begin{array}{l}\text { "Ham introduces a defined, serum-free medium able to support the clonal growth } \\
\text { of certain mammalian cells." } \\
\\
\text { "Harris and Watkins produce the first heterocaryons of mammalian cells by the } \\
\text { virus-induced fusion of human and mouse cells." }\end{array}$ \\
\hline 1968 & $\begin{array}{l}\text { "Augusti-Tocco and Sato adapt a mouse nerve cell tumor (neuroblastoma) to } \\
\text { tissue culture and isolate clones that are electrically excitable and that extend } \\
\text { nerve processes. A number of other differentiated cell lines are isolated at about } \\
\text { this time, including skeletal muscle and liver cell lines." }\end{array}$ \\
\hline 1975 & $\begin{array}{l}\text { "Köhler and Milstein produce the first monoclonal antibody-secreting } \\
\text { hybridoma cell lines." }\end{array}$ \\
\hline 1976 & $\begin{array}{l}\text { "Sato and associates publish the first of a series of papers showing that different } \\
\text { cell lines require different mixtures of hormones and growth factors to grow in } \\
\text { serum-free medium." }\end{array}$ \\
\hline 1977 & $\begin{array}{l}\text { "Wigler and Axel and their associates develop an efficient method for } \\
\text { introducing single-copy mammalian genes into cultured cells, adapting an earlier } \\
\text { method developed by Graham and van der Eb." } \\
\text { stem cells from mouse." } \\
\text { "Martin and Evans and colleagues isolate and culture pluripotent embryonic }\end{array}$ \\
\hline $1998 . "$
\end{tabular}




\subsection{Primary Cells}

Primary cells are one of the most popular choices in the in vitro studies that find a wide range of applications in various scientific disciplines. A brief summarizing description of how to isolate cells for primary cell culture of rat brain endothelial cells is described in following paragraphs $(82,83)$.

First, in the preparation stage, a suitable tissue culture flask needs to be coated with type I collagen and kept at $4^{\circ} \mathrm{C}$ overnight. After drying in the $37{ }^{\circ} \mathrm{C}$ incubator, the flask will be stored in the $4{ }^{\circ} \mathrm{C}$ fridge until use. Several different solutions will be used in the isolation procedure alone. The growth medium needs to be supplemented with various ingredients for different purposes which will be revealed in the following descriptions.

With the available appropriately euthanized rat, careful surgery procedures are applied to aseptically remove the rat brain hemispheres from the skull which will be put in PBS. Blood vessels and meninges need to be carefully removed and discarded. Then chop the brain tissue into tiny pieces using scalpels under sterile conditions. The minced brain tissue will then be put in centrifuge tubes containing $0.5 \%$ Dispase and incubate at $37^{\circ} \mathrm{C}$ for 3 hours in a shaking bath with $100-120 \mathrm{rpm}$. The mixture will be subject to centrifugation at $1000 \mathrm{~g}$ for 10 minutes, after which the pellets at the bottom will be collected and transferred to plastic bottles containing 15\% Dextran in growth medium. This mixture will undergo further centrifugation at $5800 \mathrm{~g}$ for 10 minutes to separate the microvessel from other tissue elements. The pellets were rinsed by growth medium and collected by another centrifugation. Further incubation of the collected pellets containing microvessel in growth medium and 5\% serum containing $1 \mathrm{mg} / \mathrm{ml}$ collagenase/dispase in 
a $100 \mathrm{rpm}$ shaking water bath for $14-16$ at $37^{\circ} \mathrm{C}$ will help to remove the basement membranes and pericytes. This mixture will undergo centrifugation for 6 minutes at $800 \mathrm{~g}$, followed by rinsing with growth medium and 5\% serum. Another centrifugation under of 6 minutes at $800 \mathrm{~g}$ will result in the pellet ready for next step.

The pellet solution from last step will be added onto Percoll gradient solution containing 50\% Percoll in growth medium in a centrifuge tube. Centrifuge this mixture for 10 minutes at $1000 \mathrm{~g}$ will separate the endothelial cells from cellular debris and red blood cells. Then the pellets will be resuspended with growth medium and $10 \%$ serum and centrifugation will be repeated to remove further impurities. The final density of the resuspended pellet should be controlled at around $1.055 \mathrm{~g} / \mathrm{ml}$ of cell mixtures in growth medium and $10 \%$ serum. The cell number can be determined by hemacytometer counting under an optical microscope. According to the protocol, 2-4g surgically obtained brain tissue can yield approximately $0.4-0.7 \times 10^{6}$ cells. Part of the obtained cells can be seeded on the collagen coated flask at the density of $0.5 \times 10^{6}$ cells $/ \mathrm{cm}^{2}$ and maintain growing. Rest of the cells can be cryo-preserved in liquid nitrogen for future use.

In the very initial stage of the dissertation, several trials of isolating rat brain endothelial cells were attempted. However, due to the complication of available experiment facility, as well as the limited supply of animal sources, the author was not able to successfully obtain enough amounts of isolated rat brain endothelial cells sufficient for the study. A real lab picture demonstrating the obtaining of rat brain is shown below. The bulging eyes of the rat were like a silent accusation which arouses another important ethical issue why the author was determined to use immortalized cell lines in her study. The reason will be briefly discussed in later sections. 


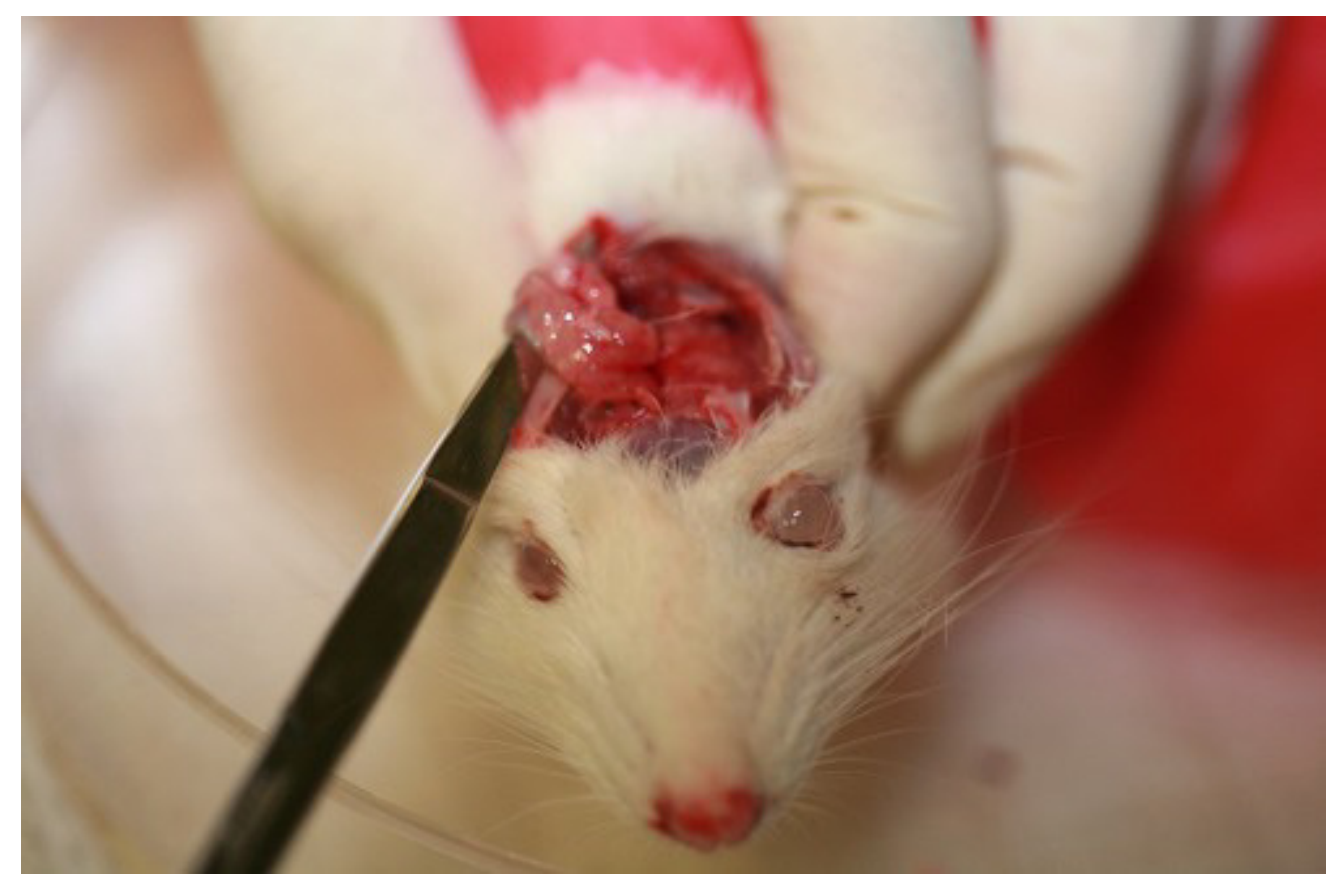

Figure 7 Dissecting rat for its brain hemispheres

\subsection{Immortalized Cell Lines}

It is not an easy task to obtain and maintain primary cells cultures, even after all those time and money consuming procedures described in the previous section. According to Dorovini-Zis et al., primary human brain endothelial cell culture generally will lose $\mathrm{BBB}$ characteristics after only after passages. While on the other hand, immortalized cell lines are able to retain certain level physiological and morphological of the BBB characteristics. And the best part is, unlike primary cell lines, immortalized cell lines are able to proliferate through an extended number of passages without going into senescence. Nowadays, biological companies are able to provide researchers with abundant ready to use high quality immortalized cell lines at relatively low prices. Immortalized cell lines are becoming more and more important yet convenient tool in cell and tissue studies. 
Immortalized cell lines are initially obtained from isolating desired cells from animal sources. However, a procedure altered these cells which made them virtually escape the cell fates and that why they are called immortalized cells. The underlying theoretical basis to immortalize cells is through expression of the telomerase reverse transcriptase protein, particularly those cells most affected by telomere length. This protein is inactive in most somatic cells, but when it is exogenously expressed the cells are able to maintain telomere lengths sufficient to avoid replicative senescence. The most popular method to alter cells is known as viral transformation. Viral genes such as adenovirus E1A and E1B, human pappiloma virus (HPV) E6 and E7, Simian virus 40 (SV 40) and Epstein-Barr virus (EBV) are among the popular ones to be used in to immortalize cells by viral transformation.

The immortalization procedure is a fairly simply procedure, and best of all, this procedure is usually accomplished by the cell vendors but not the researchers. Thus by purchasing the already immortalized cell lines, researchers are able to obtain "standardized" cells that can be passed on for an extensive number of passages which helps to save both time and money.

\subsection{Choice between Primary and Immortalized Cell Lines}

As previously introduced, co-culture systems with endothelial cells and astrocytes are by far the most widely used in vitro BBB modeling methods for drug delivery studies(6). Different cell lines including bovine brain endothelial cells, human primary brain endothelial cells, MDCK cells lines, and caco-2 cell lines have been used in the existing in vitro $\mathrm{BBB}$ models(7). However, most of the current successful $\mathrm{BBB}$ in vitro models are based on primary cell cultures. 
The reason for choosing primary cell cultures is mainly because of their relatively high correlations with in vivo models in terms of high TEER values and tight junction proteins expression. However, the seemingly high correlation inevitably diminishes within several passages of initial cultures(28). Besides, the availability of the primary cells can be limited for primary cultures because of the accessibility of the animals. Pathogens and donor-to-donor variability account for other major drawbacks of primary cell culture $(28,84)$. The repeatability and consistency of the in vitro models are thus unappealing. Last but not least, with the rising awareness of animal rights, researchers are facing an important ethical issue of repeatedly sacrificing animals for cells. Unfortunately, because of the nature of the primary cells, numerous animals will have to be sacrificed to meet the demand of new and more cells. The author herself volunteers in an animal shelter and is reluctant to use animal as she believes animals and mans are created equal; animals should not be sacrificed unlimitedly for the mere benefit of human. With this in mind, commercially available immortalized cell lines can be considered as an alternative.

Several advantages of using commercially available immortalized cell lines are: 1. reliability: cells can be purchased from trusted well-established sources; 2. consistency and reproducibility: the variance between different trials of experiments can be minimized since the cell source is controlled and consistent; 3 . longevity: immortalized cell lines are specially treated by a transfection process so that the important cell features can be maintained in a prolonged period of time $(85)$; 4 . accessibility: cells are available to be purchased at any time, waiting time for the cell source can be confidently diminished; 5. efficiency: preparation time and cost are reduced because the cells can be 
immediately used upon purchase; 6. ethically: yes animals will be sacrificed for their cells, but since the immortalized cells can be reproduced and cryo-preserved in large quantity for an extended time period without going into senescence, the number of sacrificed animals is significantly reduced in comparison to those primary culture methods that demands new cells every several weeks.

For example, in this particular study, the authors purchased both cell lines from ATCC with the total price of under $\$ 500$. Upon receiving, the cells were cultured in appropriate ways to expand the numbers and most of them were later cryo-preserved for future use. The cells used in the assessment were from passage 2 to passage 10 . No significant difference was observed between samples made from different passages of the cells, thus the consistency of the in vitro samples was made possible. If the authors chose to use immortalized animals, for each type of the cell, 2 to 3 days would have to be dedicated into cell isolation. The cost for all the various chemicals may add up to several hundred dollars. Rat would be a favorable choice of animal since they are relatively easier to obtain and lower costly, but each rat can still cost from $\$ 20$ to nearly $\$ 100$, let alone the special trained personnel needed to handle the animal. Even if the primary cells were successfully isolated and expanded, another animal will have to be sacrificed before the passage number reach 10 . There could be variance between each passage, and also between animals. The efficiency difference between primary cells and immortalized cell lines is apparent. To the author's best knowledge, no group has reported successful results from in vitro BBB models with aECM using commercially available immortalized cell lines. It will be very interesting to see how the results in this dissertation compared to traditional in vitro models. 


\subsection{Summary}

This short chapter starts with the introduction of noticeable events/discoveries in the cell and tissue culture history that every tissue engineer should come to acknowledge and appreciate. The table presented by NCBI summarizes the milestones in the tissue engineering history, which helps the author better understand and more proper handling of tissue culture experiments nowadays. After that, primary and immortalized cell cultures are introduced with an example of how to obtain and isolate primary cells from an animal. Then at the end of the chapter, the comparisons between these two cell culture methods are made and author's decision and reasoning are stated. 


\section{CHAPTER V ENGINEERING IN VITRO BBB MODEL}

\subsection{Existing BBB in vitro Models}

\subsubsection{Early Attempts}

Primary cultures were the main focus in the earlier attempt on a blood-brain barrier in vitro model. Endothelial cells isolated from porcine, bovine and rodent brains were established into in vitro cultures. The main reason for setting up those systems was to test in vitro how well the drugs can penetrate through the barrier since in vivo test was difficult to perform. As the result, the systems did show some transport properties close to the real cases, and junctions were formed. But the TEER values were not high at all falling in the range of around $10 \mathrm{~cm}^{2}$ which is similar from the endothelial cells from aorta. The results were not surprising, but they were clearly not too successful as an in vitro model for valid tests.

Other early attempts have been focusing on testing and understanding the properties of the endothelial cells and capillaries on blood-brain barrier in vitro. Thus a system very similar to the brain needed to be derived. Scientists have tried on endothelial cells isolated from porcine, rodent and even human brain tissues. Some of the data or results discussed on the $\mathrm{BBB}$ features are thanked to those experiments.

\subsubsection{Current Attempts}

It has been mentioned in previous chapters that the current most popular method is to establish the in vitro $\mathrm{BBB}$ models with the presence of astrocytes trying to adjust the culturing environment to be more like in the brain. Numerous studies have been carried out by various groups of scientists, and some did show promising results on several of the BBB features including tight junction, high resistance, as well as the expression of some 
BBB definitive substances such as the expression of P-glycoprotein. Some examples reviewed and reproduced by Garberg et al.(7) are briefly introduced in the next section.

\subsubsection{Primary Bovine Brain Endothelial Cells (BBEC)}

This was not a real co-culture case. Primary BBECs (from passage 4-7) were cultured on the upper side of a cell culture insert (Millicell PC $3 \mu \mathrm{m}$ pore size, $30 \mathrm{~mm}$ diameter, by MIllicell Corporation Bedford, MA 01730) with primary rat astrocytes cultured on the bottom of the same well where the insert was. The media for the coculture system was conditioned and modified for both cells' optimal growth. The endothelial cells layer formed in 7 days. This model showed high TEER value (500-800 $\Omega$ $\left.\mathrm{cm}^{2}\right)$ with comparably low permeability to two tested markers: sucrose $\left(4.0^{*} 10^{-6} \mathrm{~cm} / \mathrm{s}\right)$ and insulin $\left(0.7^{*} 10^{-6} \mathrm{~cm} / \mathrm{s}\right)$.

\subsubsection{Primary Human Brain Endothelial Cells (HPBEC)}

This is a co-cultured in vitro model where astrocytes were plated on collagen coated Transwell ${ }^{\mathrm{R}}$ inserts $(0.4 \mu \mathrm{m}$ pore size, $12 \mathrm{~mm}$ diameter by Costar) until reaching confluency, followed by endothelial cells plating on the other side. Primary HPBEC were established from adult human normal tissue and primary astrocytes were from normal human cerebral tissue. The endothelial cells layer formed in 15 days. A series of transport tests of various agents were performed on this co-culture model. However, the results did not succeed in telling apart between transport mechanisms between passive transport and carrier mediated transport or active transport.

\subsubsection{Immortalized Rat Brain Endothelial Cells (SV-ARBEC)}

The SV-transfected immortalized ARBECs were cultured with conditioned media by SV-transfected immortalized neonatal rat astrocytes. The culture vessel was collagen 
coated cell inserts $\left(1 \mu \mathrm{m}\right.$ pore size, $0.83 \mathrm{~cm}^{2}$ growth area by Falcon). The researchers established the immortalized cell lines by SV-transfection, a popular way for immortalization. After lengthy transfection procedures, SV-ARBECs from passage 80-90 and SV-astrocytes from passage 75-85 were used in this study. The astrocytes conditioned media were collected after 4 days from an $80 \%$ confluent astrocytes culture. However, the results of transport study were confusing. For example, instead of being influxed, some testing compounds were found to be effluxed by the in vitro model. And for small molecules such as caffeine, carrier mediated transport were found to be more dominant than passive transport, which was controversy to the theories. What's more, it was impossible to find the correlation between in vivo and in vitro permeability coefficients.

\subsubsection{Immortalized Mouse Brain Endothelial Cells (MBEC4)}

This is not a co-culture system: the authors immortalized MBEC4s by SV 40 and then cultured the cells by themselves on $\operatorname{Transwell}^{\mathrm{R}}$ cell culture inserts $(0.4 \mu \mathrm{m}$ pore size, for 12 -well plates). The transport study was conducted 40 hours after the monolayer seeding, and TEER measurement took place just 30 minutes after the cells were seeded. Transport polarity on the membrane when tested from basolateral to apical and apical to basolateral was not distinguished. Not too many valuable data was observed from this model. Similar as for SV-ARBEC the case, not surprisingly, in vivo and in vitro permeability coefficients were also low.

\subsubsection{Madin-Darby Canine Kidney Cell Lines(MDCK)}

MDCK have been reported as blood-brain barrier models(86). In this, three MDCK cell lines were studied: one cell line was immortalized MDCK purchased from 
ATCC, and the other two were provided by Netherlands Cancer Institutes. The MDCKs were cultured on Transwell $^{\mathrm{R}}$ cell culture inserts $(0.4 \mu \mathrm{m}$ pore size, $12 \mathrm{~mm}$ diameter, by Costar, Cambridge, UK) growing with media with necessary supplements but not conditioned by astrocytes. TEER values were carried out by a "chopstick electrode" (by Millicell ERS, Millipore, Oslo, Norway). MDCK from ATCC (from passage 59-63) had TEER value measured at about $30 \mathrm{~cm}^{2}$. And it did show some ability in detecting passively distributed substance during the study. TEER values for the other two MDCK cell lines (from passages 8-23) were even lower than MDCK from ATCC. But they also did show the ability in distinguishing compounds for passive transport and know efflux substrate.

\subsubsection{ECV304 Cell Line}

Previous research reported ECV304s were used for feasible immortalized bloodbrain barrier models $(44,87,88)$. In this study, scientists used immortalized ECV304 and rat glioma (C6) both purchased from European Collection of Animal Cell Cultures (ECACC). Cellagen ${ }^{\mathrm{TM}}$ membrane culture inserts were used in this experiment. ECV304 cells were cultured on the upper side of the inserts, and C6 glioma cells were cultured on the other side of the inserts. Confluent endothelial cells layers were achieved after 3-4 days of initial plating. The highest TEER values at around $10 \Omega \mathrm{cm}{ }^{2}$ were observed at within 5-6 after endothelial cells were seeded. Relatively high correlation between in vivo and in vitro permeability was reported from this model.

\subsubsection{Human Colon Adenoma Derived Cell Line (Caco-2)}

Caco-2 cell line has become a very good example predicting intestinal absorption(89). Yee and Artursson et al. reported correlation the Caco-2 cell line with in 
vivo absorption in human $(90,91)$. But it may not be an ideal cell line in prediction of blood-brain barrier tight junction(92). In the author's study, the immortalized Caco-2 cell line was purchased from ATCC. They were cultured on a Transwell ${ }^{\mathrm{R}}$ insert's $(0.4 \mu \mathrm{m}$ pore size, $12 \mathrm{~mm}$ diameter) upper side. Transport studies were carried out within 14-28 after the cells were seeded. TEER values were carried out by using a "chopstick electrode" (by Millicell ERS, Millipore, Oslo, Norway). Actually, this is not a real bloodbrain barrier model. Rather, it should be called a research focusing on monolayer's transport properties. However, the test results showed relatively high TEER value $\left(>600 \Omega \mathrm{cm}^{2}\right)$ for this cell line and low permeability for some of the testing compound. But the in vivo and in vitro are still reported to be poorly correlated.

\subsubsection{Other Approaches}

Scientists have also tried alternative measures by directly using approved factors to induce endothelial tight junctions and $\mathrm{BBB}$ characteristics in the in vitro models. It was reported by Rubin et al. in 1991 that the endothelial tight junction and resistance showed remarkable increment with raised level of cyclic adenosine monophosphate (cAMP)(93). Several other researchers have reported similar results using the same method $(70,94,95)$. This endeavor has provided optional route building the endothelium alone. But the ultimate goal in this specific dissertation will be to establish and mimic a system actually close to the real brain, so the endothelial cells alone will not be sufficient enough. Furthermore, another important aspect of the particular study will be to evaluate the efficacy of using commercially available immortalized cell lines to improve efficiency of the modeling work. Thus a co-culture in vitro model built from purchased cell lines with the addition of astrocytes derived aECM will be the focus of study. 


\subsection{Materials and Reagents}

\subsubsection{Choice of Cell Lines}

In this study, two immortalized cell lines purchased from American Type Culture Company (ATCC, Manassas, VA) are employed in the in vitro co-culture system. They are mus musculus (mouse) endothelial cells (CRL-2583) and Rattus norvegicus (rat) astrocytes (CRL-2006). Brief introduction of the cell lines by ATCC is as following paragraphs show.

The C166 cell line was established from cells from a 12-day F1 embryos obtained by mating a female NMRI/GSF mouse with a male CD-1 mouse that was transgenic for the human fes (fps/fes) proto-oncogene. C166 cells exhibit normal endothelial characteristics, such as rearrangement into tube like structures when placed on Matrigel $^{\circledR}$ and retention of cobblestone morphology at confluence. The cell line was reported with capability to support the stable proliferation of multipotent hematopoietic stem cells, thus generating adequate numbers of cells for study of the mechanisms involved in their subsequent development and differentiation. The CRL-2583 cell line which will be actually used in the study was derived from the C166 cell line by transfection with a plasmid reporter vector, pEGFP-N1, encoding enhanced green fluorescent protein (GFP).

Type 1 rat astrocytes/glial cells have been reported to successfully inducing various sourced endothelial cells some of the blood-brain barrier phenotype and characteristics by researchers. The CRL-2006 cell line used in this study was established from primary cultures of type 1 astrocytes from brain frontal cortex tissue of 1 day old rats. The cells are reported to retain characteristics consistent with the phenotype of type 
1 astrocytes. About $20 \%$ of the cells have glial fibrillary acidic protein (GFAP) immunoreactivity.

Another relevant reason to choose these two specific cell lines is because they are both recommended by ATCC to grow in the same type of medium. Thus the time of handling and complexity of the experiment can be further reduced. Because if in the coculture, the two types of cell lines require different media, usually media conditioning will be required to finally find a optimal media mix ratio for the health of both of the cell lines, which can take more than one month and more than 5 passages to accomplish.

\subsubsection{Choice of Culture Vessels}

1) $\mathrm{BD}^{\mathrm{TM}}$ Falcon canted neck tissue culture flasks with filter caps: major culture vessel to proliferate cells in order to provide enough number of cells for further study. Two different sizes $25 \mathrm{~cm}^{2}$ and $75 \mathrm{~cm}^{2}$ satisfy the demands of cell numbers. The canted neck with filter caps help to reduce contamination during handling.

2) Corning Costar ${ }^{\circledR}$ Collagen I \& III coated (COL) Transwell ${ }^{R}$ inserts for $24-w e l l$ plates: major cell culture vessels for building co-culture system. The two types of cells will be seeded on the two sides of the insert, respectively. The inserts feature polytetrafluoroethylene (PTFE, commercial name: Teflon) membrane coated with rat tail collagen I \& III with $0.4 \mu \mathrm{m}$ pore size ideal for co-culturing.

3) Corning $\operatorname{Costar}^{\mathbb{B}}$ Transwell $^{\mathrm{R}}$ inserts for 24 -well plates: cell culture vessels for building co-culture system alongside the COL inserts. These inserts have similar specifications as for the COL inserts expect for the membrane material and collagen coating. Table 3 shows some most important specification of the two types of inserts. 
Table 3 Specifications of Corning Costar ${ }^{\circledR}$ Transwell $^{\mathrm{R}}$ inserts for 24-well plates

\begin{tabular}{|l|l|l|}
\hline Insert type & COL Transwell & Normal Transwell \\
\hline Insert membrane material & PTFE & Polycarbonate \\
\hline Coating & Type I \& III collagen & None \\
\hline Optical property & Clear when wet & Translucent \\
\hline Cell visibility & Cell outlines & Poor \\
\hline Pore size ( $\mu \mathrm{m})$ & 0.4 & 0.4 \\
\hline Insert diameter (mm) & 6.5 & 6.5 \\
\hline Insert Growth area (mm $\left.{ }^{2}\right)$ & 0.33 & 0.33 \\
\hline Media volume $(\mu \mathrm{l} /$ well) & 600 & 600 \\
\hline Seeding density (cells/well) & 30000 & 30000 \\
\hline Media volume ( $\mu \mathrm{l} /$ insert) & 100 & 5000 \\
\hline Seeding density (cells/insert) & 5000 & \\
\hline
\end{tabular}

4) Corning Costar ${ }^{\circledR}$ multi-well plates: major cell culture vessels for testing, where the inserts are placed. The 24-well plates are the major vessel in the co-culture model building to host the inserts. While the 12-well plates are used to produce astrocytes derived aECMs.

5) Nalgene ${ }^{\mathrm{TM}}$ Cryogenic vials: major vessel to preserve excessive cells in liquid nitrogen vapor phase for further use. The $1.5 \mathrm{ml}$ volume is suitable for the cryopreservation system in the current lab.

6) Corning Pyrex Brand Crystallizing Dishes: it is a special tool to be use to host the inverted culture inserts to allow cells grow on the bottom side of the inserts. The 
specific model has a height of 1.96 inch, high enough for the inverted inserts to be totally submerged while still having enough space on top of the inserts to hold the media. More details about this deep dish will be introduced in the sample setup section.

\subsubsection{Media and Other Major Reagents}

1) Dulbecco's Modified Eagle's Medium (DMEM) from ATCC: it is the recommended cell culture media for both cell lines in this study. The medium contains 4mM L-glutamine, $4500 \mathrm{mg} / \mathrm{L}$ glucose, $1 \mathrm{mM}$ sodium pyruvate, and $1500 \mathrm{mg} / \mathrm{L}$ sodium bicarbonate. The DMEM provides optimized energy sources for protein production and nucleic acid metabolism while limiting toxic ammonia build-up. It is to be used with 5\% $\mathrm{CO}_{2}$ to maintain $\mathrm{pH}$. The DMEM contains phenol red to indicate the $\mathrm{pH}$ change in the medium. Its color exhibits a gradual transition from yellow to red over the $\mathrm{pH}$ range 6.6 to 8.0. Phenol red will turn bright pink when the whole solution's $\mathrm{pH}$ is $>8.1$.

2) Fetal bovine serum (FBS) from HyClone ${ }^{\mathrm{TM}}$ (Logan, UT): it is the major supplement $(10 \% \mathrm{v} / \mathrm{v})$ of the cell culture medium as recommended from ATCC. It contains mostly proteins but also growth factors, hormones, amino acids, sugars, trypsin inhibitors, and lipids. The serum was defined for optimized performance and all the sera are from the same lot to keep the consistency throughout the whole research period.

3) Penicillin-Streptomycin-Glutamine from $\mathrm{GIBCO}^{\mathrm{TM}}$ : it is the recommended supplement $(1 \% \mathrm{v} / \mathrm{v})$ as antibiotics in the cell growth medium. Its function in the medium is to lower the risk of contamination in the cell culture.

4) Dimethyl sulfoxide (DMSO): it is the recommended supplement $(5 \% \mathrm{v} / \mathrm{v})$ in the complete culture medium to construct cryoprotectant medium. The function of 
DMSO in the cryoprotectant medium is to prevent cell membrane rupture from avoiding crystallization of liquid in cells when they are cryopreserved.

5) Trypsin/EDTA from GIBCO $^{\mathrm{TM}}$ : it is the main reagent used to enzymatically detach and re-suspend cells on the cell culture vessels for harvesting and sub-culturing. Trypsin is a serine protease found in the digestive system, where it breaks down proteins. It is involved in numerous bio-technological processes. Actually, Trypsin/EDTA is a combined method for detaching cells while trypsin cuts the adhesion proteins in cell-cell and cell-matrix interactions, and EDTA acts as a calcium chelator, 'mops' up the remaining divalent cations for the better performance of trypsin. $0.25 \%$ Trypsin/EDTA is used in this study as recommended.

6) Dulbecco's Phosphate Buffered Saline (PBS) from GIBCO $^{\mathrm{TM}}$ : PBS is a buffer solution commonly used in biochemistry. It is a salty solution containing sodium chloride, sodium phosphate and potassium phosphate. In this study, the PBS contains no calcium or magnesium. It is usually used to rinse the cell layer after the removal of culture medium and before application of Trypsin/EDTA to minimize the effect of the trypsin inhibitor in the old medium. It is also used to rinse the cell layer after removal of culture medium when there are more than normal amount of dead cells floating in the culture or for any other reasons that require a "medium free" cell layer.

7) Urea from Aldrich $^{\mathrm{TM}}$ : Urea is an organic compound of carbon, nitrogen, oxygen and hydrogen, with the formula $\mathrm{CON}_{2} \mathrm{H}_{4}$ or $\left(\mathrm{NH}_{2}\right)_{2} \mathrm{CO}$. In this study, a $0.5 \mathrm{M}$ urea solution will be used to lyse the astrocytes monolayer for aECM as a gentle lysis solution, which can kill the cells themselves but leaving the extracellular component that astrocytes secreted behind. 
8) Stericup $0.22 \mu \mathrm{m}$ filter units from Millipore (Billerica, MA): the vacuum filtration system includes a filter unit together with a receiver flask which can later serve as container for the filtered liquid. The $0.22 \mu \mathrm{m}$ pore size on the filter membrane is suitable to remove bacteria and particle that may cause contaminations in the cell culture. This system is used to filter the supplemented media and other solutions that need sterilization in the experiments.

\subsubsection{Other Equipments and Lab Wares}

Major equipments are: bio-safety laminar flow hood, incubator, chemical flow hood, centrifuge machines, lyophilizer, phase contrast microscope, fluorescent microscope, scanning electric microscope, confocal microscope, TECAN micro plate optical reader, TEER measurements chambers etc. These specific instruments will be mentioned respectively where they are used.

Major labware are: electric pipettor, various pipettes, pipettor, matching pipet tips, multi-channel pipettor, matching pipet tips, polypropylene conical centrifuge tubes, forceps, Hemacytometer, etc.

\subsection{Engineering Astrocytes Derived aECM}

\subsubsection{Theoretical Basis}

It has been acknowledged that cells produce and interact with their extracellular components in an organized matrix better known as $\operatorname{ECM}(96)$. In the central nervous system, the ECM is located between astrocytes and cerebral capillaries(97). This ECM is reported to provide structural support as well as signaling directions for brain microvascular endothelial cells(97). There's proof that bovine aortic endothelial cells cultures on glial ECM showed dramatically higher activity of $\gamma$-GTP than those cultured 
on normal vessels(97). In the construction of the in vitro blood-brain barrier model, it would be beneficial to observe the interaction between endothelial cells and the ECM created by astrocytes which will probably lead to a more successful way of establishing the particular model.

\subsubsection{Methods}

A layer of acellular extracellular matrix (aECM) will be prepared according to the protocol by Mizuguchi et al. (97). In the beginning, rat astrocytes were seeded at the density of $2 \times 10^{4}$ cells/well on a 12 -well plate. The culture media (fully supplemented DMEM) was changed daily in order to achieve the best proliferation, especially when the cells were getting confluent. The cells were kept growing for a consecutive 10-day period until full confluency was reached. On the last day, the media was removed and the confluent cell layers were rinsed with cold PBS twice. Then the cells were incubated with $0.5 \mathrm{M}$ urea for 10 minutes to lyse the cells at room temperature. After the treatment, the layer was decellularized and ready to be used as aECM in the in vitro model. The resulting aECM was rinsed with PBS carefully 10 times to remove cell debris as well as the urea residues. The aECM could be kept soaked in PBS and stored in the fridge for up to two weeks.

According to ATCC, the confluent astrocyte cell layer was found to "come off the culture surface in a sheet" and difficult to dissociate in previous studies(98). The phenomenon was consistent with the observation in the experiment. At the end of the 10day culturing period, a layer of confluent astrocytes was formed. This cell layer was resilient and could even endure normal the trypsinization procedure without breaking up. This feature was fully utilized in this specific research to successfully produce the aECM. 
Figure 8 shows an example of the automatically delaminated confluent astrocyte layer. Such a confluent layer was a homogeneous sheet full of astrocyte cell bodies providing an ideal scaffolding substrate when decellularized for endothelial cells seeding.

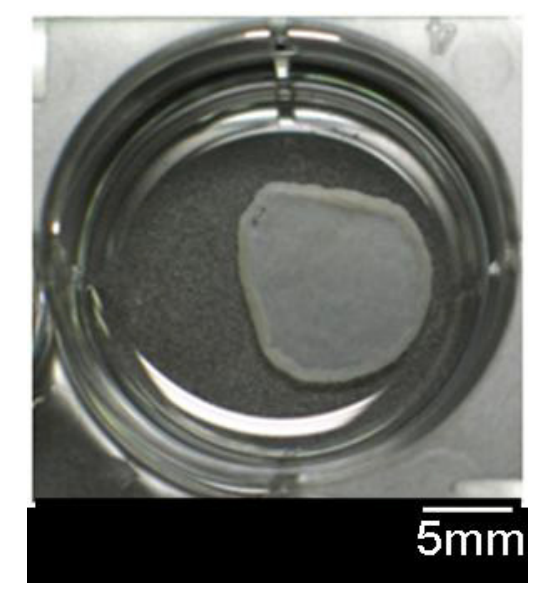

Figure 8 Delaminated confluent astrocytes layer

\subsubsection{Scanning Microscopy (SEM)}

At the end of the 10-day culturing period, selected confluent astrocytes layer and the decellularized aECM were prepared for SEM examination. Briefly, samples were fixed with $10 \%$ glutaraldehyde (Sigma-Aldrich, St. Louis, MO) overnight; the fixed samples were then serially dried by $10 \%, 30 \%, 50 \%, 75 \%, 90 \%$ and $100 \%$ ethanol (Fisher Scientific, Pittsburgh, PA). After the last drying, the samples were treated with hexamethyldisilazane (HMDS) (Sigma-Aldrich, St. Louis, MO) for 3 min. Then, these samples were mounted onto conductive carbon tabs and freeze-dried in the lyophilizer (Labconco FreeZone Plus Freeze Dry Systems, 6L. Kansas City, MO) overnight. Final samples were examined by JEOL JSM-6335 scanning electron microscope(JOEL Ltd., Tokyo, Japan). 

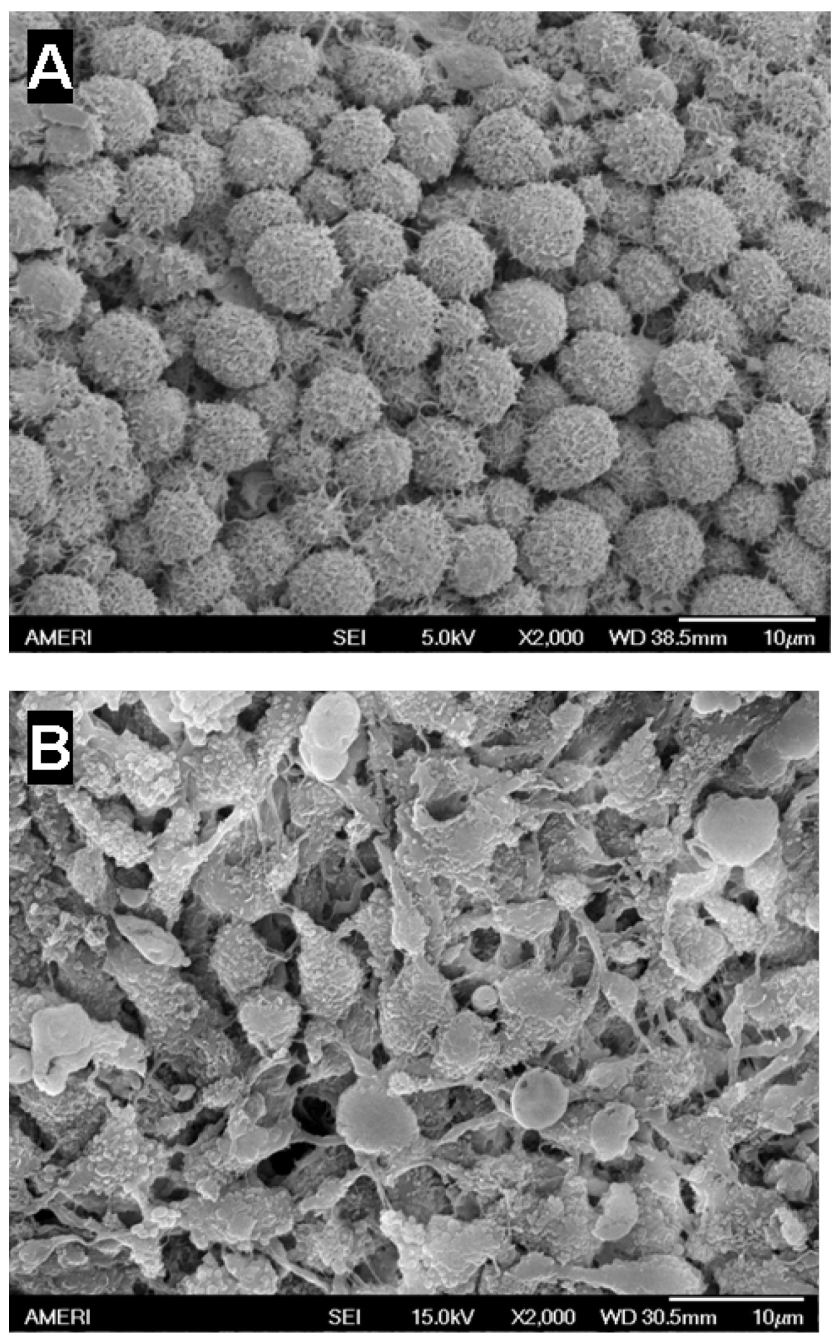

Figure 9 SEM picture of confluent astrocyte layer and decellularized astrocytes

\section{layer at the magnification of $\times \mathbf{2 0 0 0}$}

Figure 9 compares the distinguished SEM images at x2000 magnification for normal and the decellularized astrocytes layer, respectively. The normal astrocytes in (A) on the left appeared to be rounder and the microvilli were visible. From a closer look as in Figure 10, the morphology of the astrocytes at the magnification of $\times 2000$ was very similar to the commercial image example demonstrated by "Dennis Kunkel Microscopy, Inc." as Figure 11 shows. The characteristic endfeet of the astrocytes were clearly visible. 


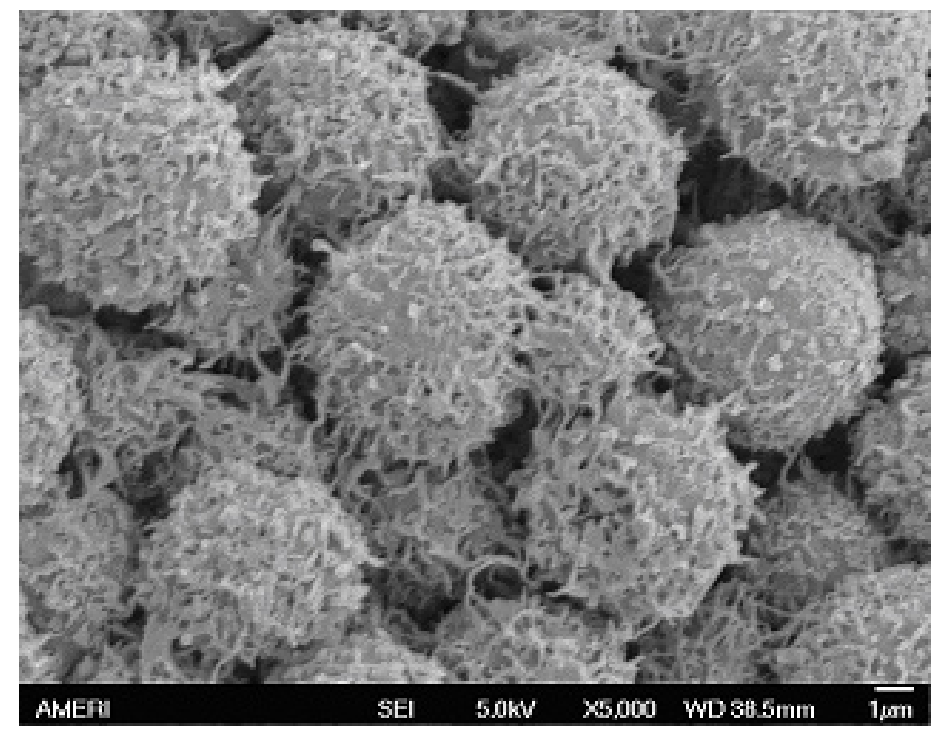

Figure 10 SEM image of astrocytes at the magnification of $\times 5000$

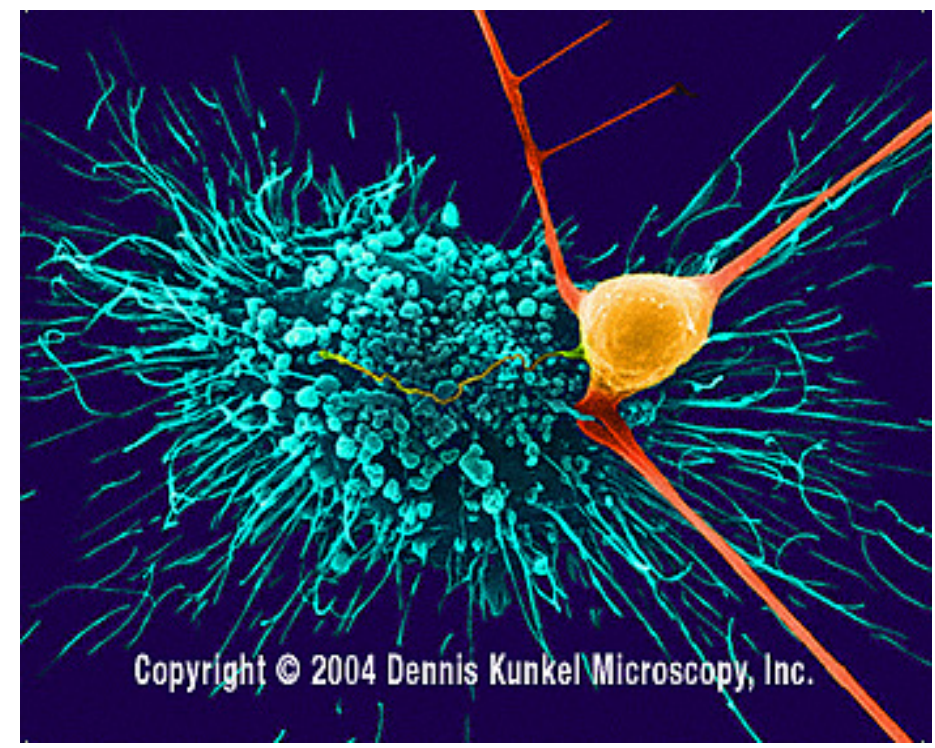

Figure 11 Demonstration of astrocyte single cell in SEM, commercially available

from Dennis Kunkel Microscopy, Inc.

As clearly showed in (B) in Figure 9, after being decellularized by the lysis solution, the aECM looked different from the normal confluent astrocytes sheet. More hollow spaces were observed for the decellularized astrocyte sheet demonstrating the washed away dead cell bodies. However, the hollow space would be ideal to serve as the 
structural support for the endothelial cells seeding. The remaining extracellular components from the astrocytes would also provide the endothelial cells a better biomimetic of the basement membrane found in the real brain.

\subsection{Culture Design}

\subsubsection{Sample Setups}

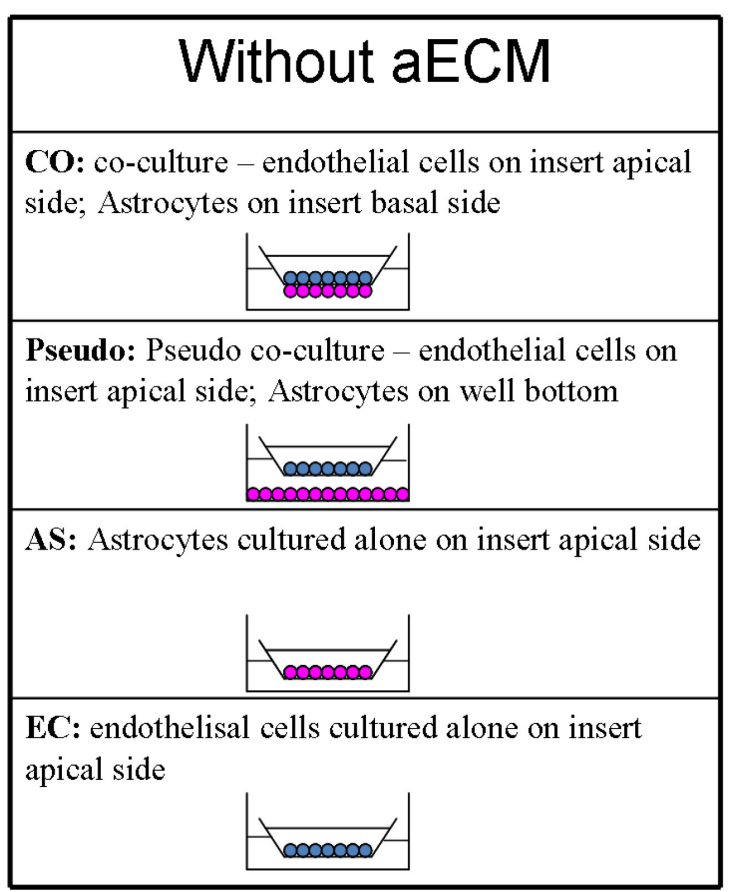

\section{With aECM}

CO+aECM: Astrocytes on insert basal side; endothelial cells on aECM on insert apical side

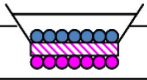

EC+aECM: endothelial cells on aECM on insert apical side

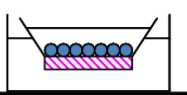

- Endothelial cells

- Astrocytes

$\mathrm{aECM}$

\section{Figure 12 Schematic demonstrations of selected sample setups in the study}

As the author's goal was to establish an in vitro co-culture BBB model with astrocyte derived aECM utilizing commercially available cell, in a total of 12 trials, various setups were attempted to compare and evaluate the efficiency in order to determine the optimal design. Some of the different sample setups are shown in schematics drawings as Figure 12 shows. How the different samples were built has been briefly described in the drawings. How the co-culture models were engineered will be introduced with details in the next section. 
Upon the arrival of the astrocytes and endothelial cells from ATCC, the cells were cultured on T-75 flasks to proliferate, respectively. After 5 days of incubation, cells on each flask were trypsinized by $0.05 \%$ Trypsin/EDTA into cells suspensions. The cell suspensions were centrifuged at $1500 \mathrm{RPM}$ for 3 minutes in room temperature. The pellets were resuspended with the freezing media (culture media containing 5\% DMSO) and distributed into $1 \mathrm{ml}$ cryo-vials under aseptic environment. The cryo-vials were first frozen at $-80^{\circ} \mathrm{C}$ for 24 hours and then transferred into liquid nitrogen cell preservative tank for future use. 20 vials of each cell type were produced in the aforementioned procedure, with roughly $200 \times 10^{4}$ cells/vial. All the subsequent experiments were carried out using the cells from the vials and the passage numbers were all within 10.

\subsubsection{Engineering the Co-Culture Samples}

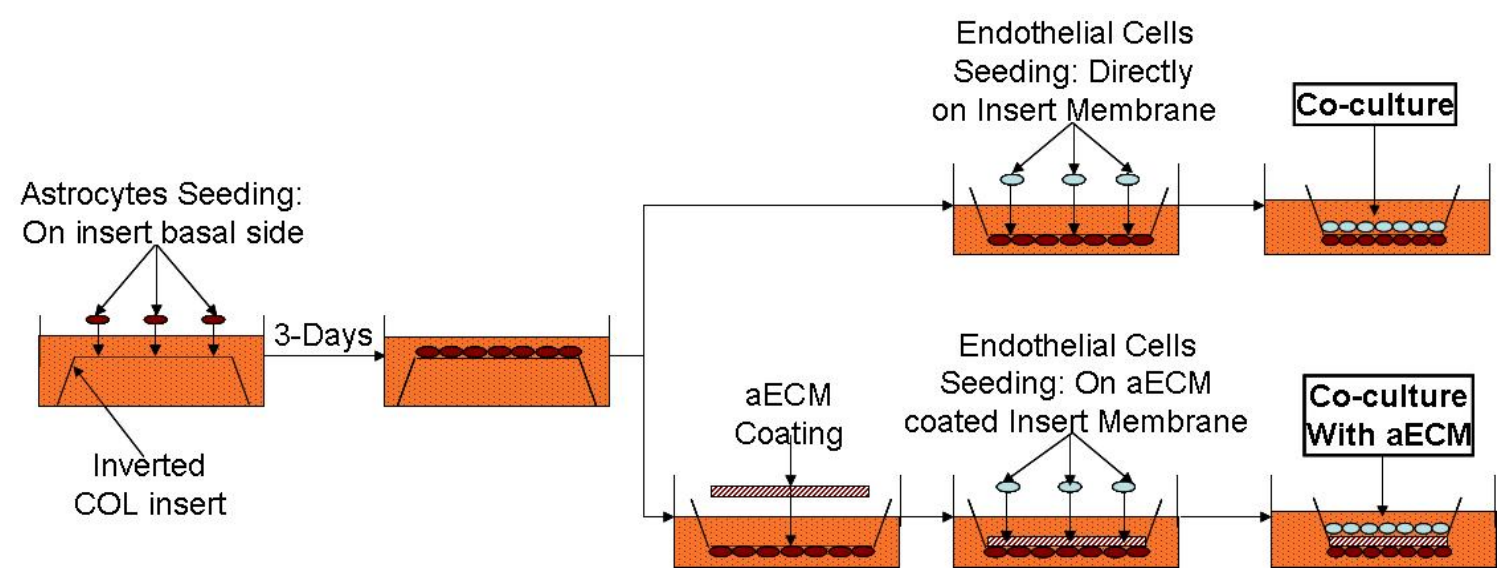

\section{Figure 13 Schematic drawing of steps in building "CO" and "CO+aECM" samples}

Figure 13 shows how the co-culture models were built on the COL inserts. In the beginning of culture period, the inserts were inverted with basal sides facing upwards and placed in a deep glass dish. Astrocytes were seeded onto the basal sides at the concentration of 5,000 astrocytes/insert. Culture media was carefully added into the deep dish in order not to wash away the just seeded cells. The deep dish was kept untouched in 
the laminar flow hood for 30 -60 minutes before being carefully transferred into the incubator. Figure 14 demonstrated how the inverted seeding looked like in real life. The astrocytes were incubated at $37^{\circ} \mathrm{C}$ in an atmosphere with $5 \% \mathrm{CO}_{2}$ for 3 days. Figure 16shows how the inverted inserts were incubated. In order to eliminate contamination, a piece of Kimwipe $^{\circledR}$ (Fisher Scientific) was loosely covered on the deep dish and secured by a round glass plate. The glass plate had a unique shape that allowed air exchange for the cells in the deep dish.

After three days of incubation, the astrocytes were assumed to attach to the basal sides of the inserts and the inserts were inverted to their original orientation and placed into the 24-well plates. For the CO setup, 5,000 endothelial cells were seeded onto each inserts' apical sides. For the CO+aECM setup, a piece of aECM produced as previously described, was placed into each insert cup and then 5,000 endothelial cells were seeded on top of the aECM. Following manufacture's protocol, the media volume for the upper chambers (insert apical sides) and lower chambers (insert basal sides) were $100 \mu 1$ and $600 \mu 1$, respectively. All the plates were incubated at $37^{\circ} \mathrm{C}$ in an atmosphere with $5 \%$ $\mathrm{CO}_{2}$. Figure 15 demonstrates the sample setups in the well plates.

For every trial, several different setups were attempted with at least 4 replications for each different setup. For those cells that did not require astrocytes seeding on the basal sides of the inserts, cells would be seeded on the apical sides of the inserts the same time when endothelial cells for the co-culture samples were seeded at the seeding density of 5,000 cells/insert. 


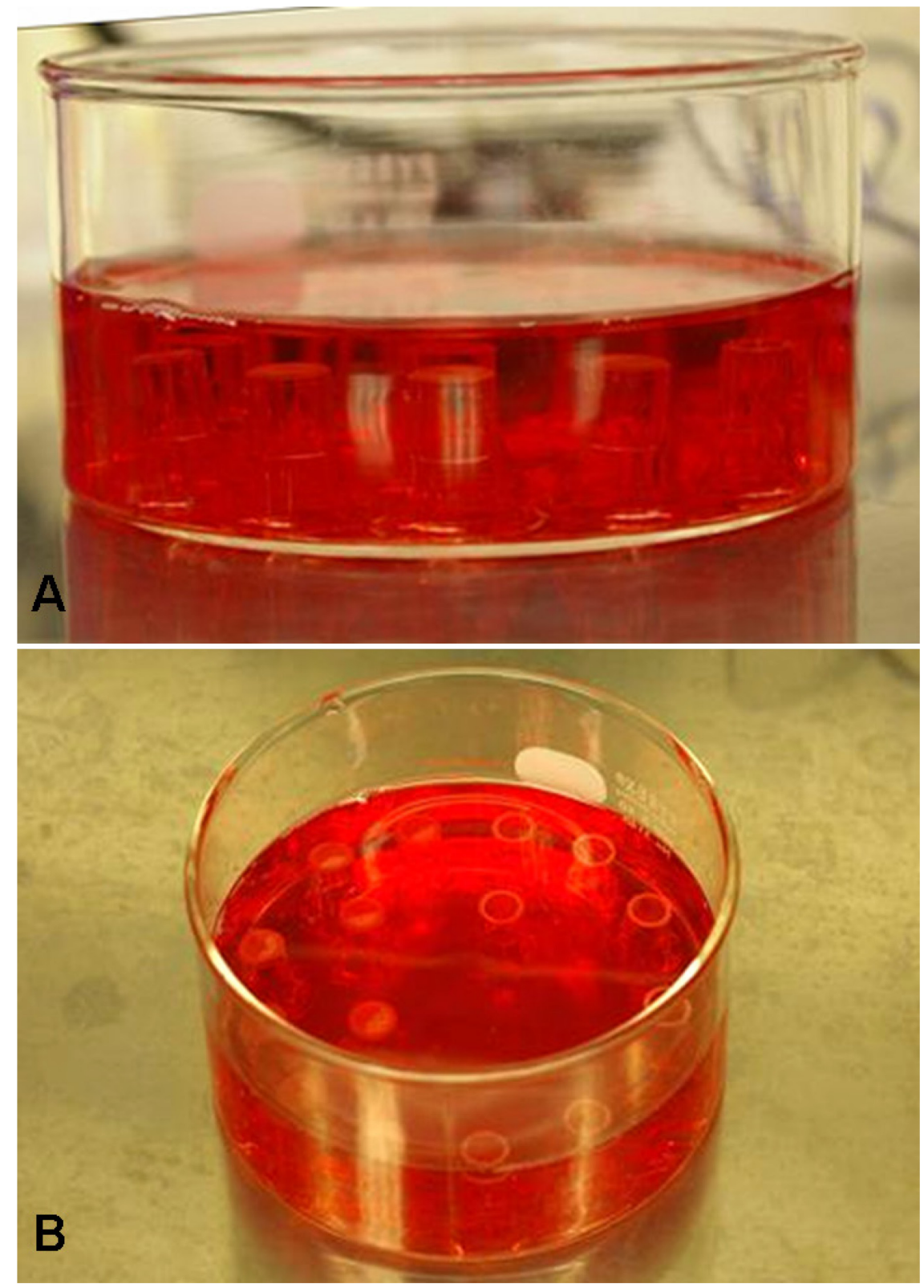

Figure 14 Astrocytes seeded on inverted inserts in the deep dish. A: side view; B: top view

For the "Pseudo" setup where astrocytes grew on the bottoms of the wells while endothelial cells grew on the apical sides of the inserts, the astrocytes were seeded at the seeding density of 10,000 cells/well 3 days prior to the endothelial cells seeding. 


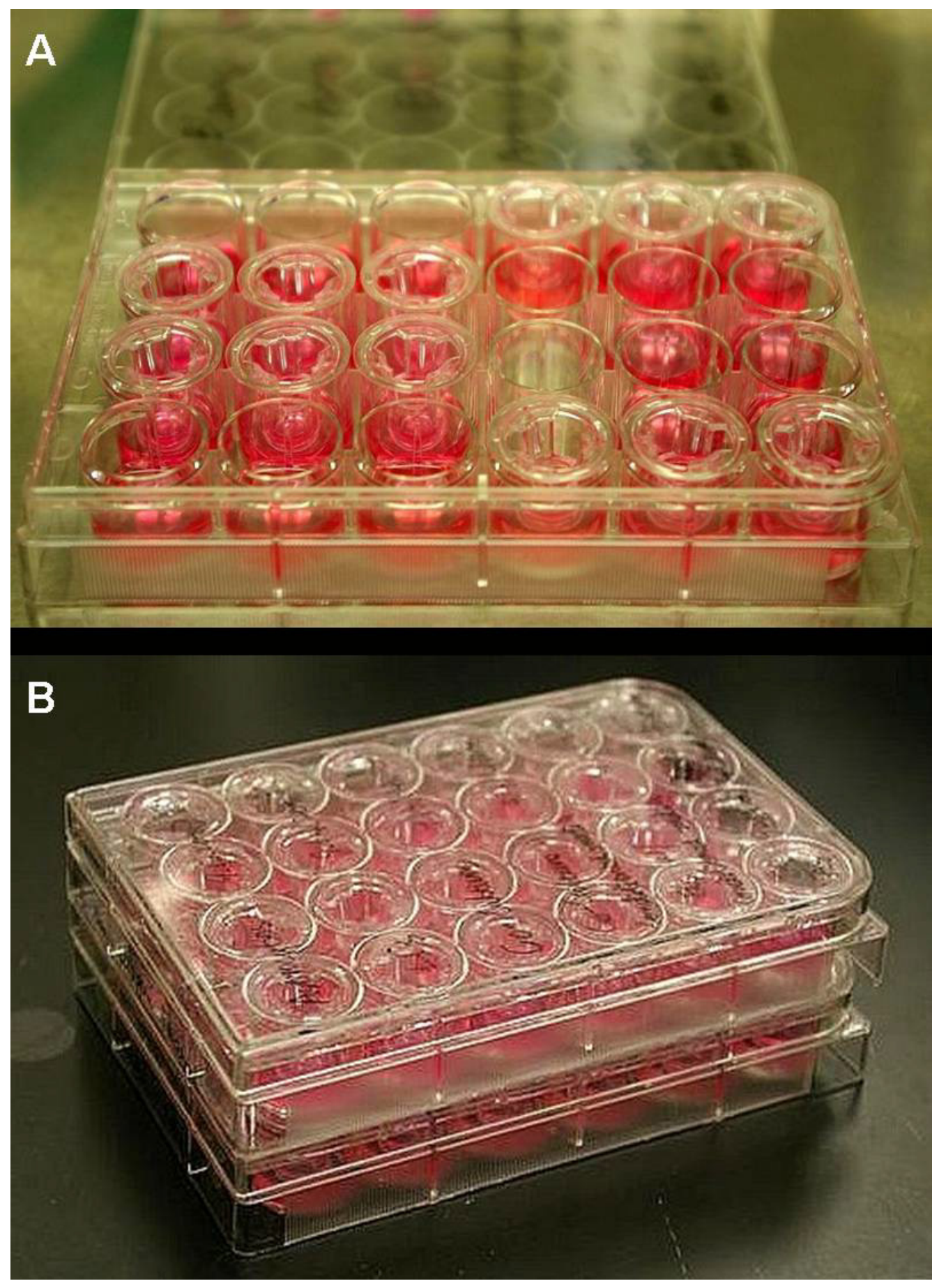

Figure 15 Sample plates. A: demonstration of how the inserts situated in the well plates. B: one batch of samples 


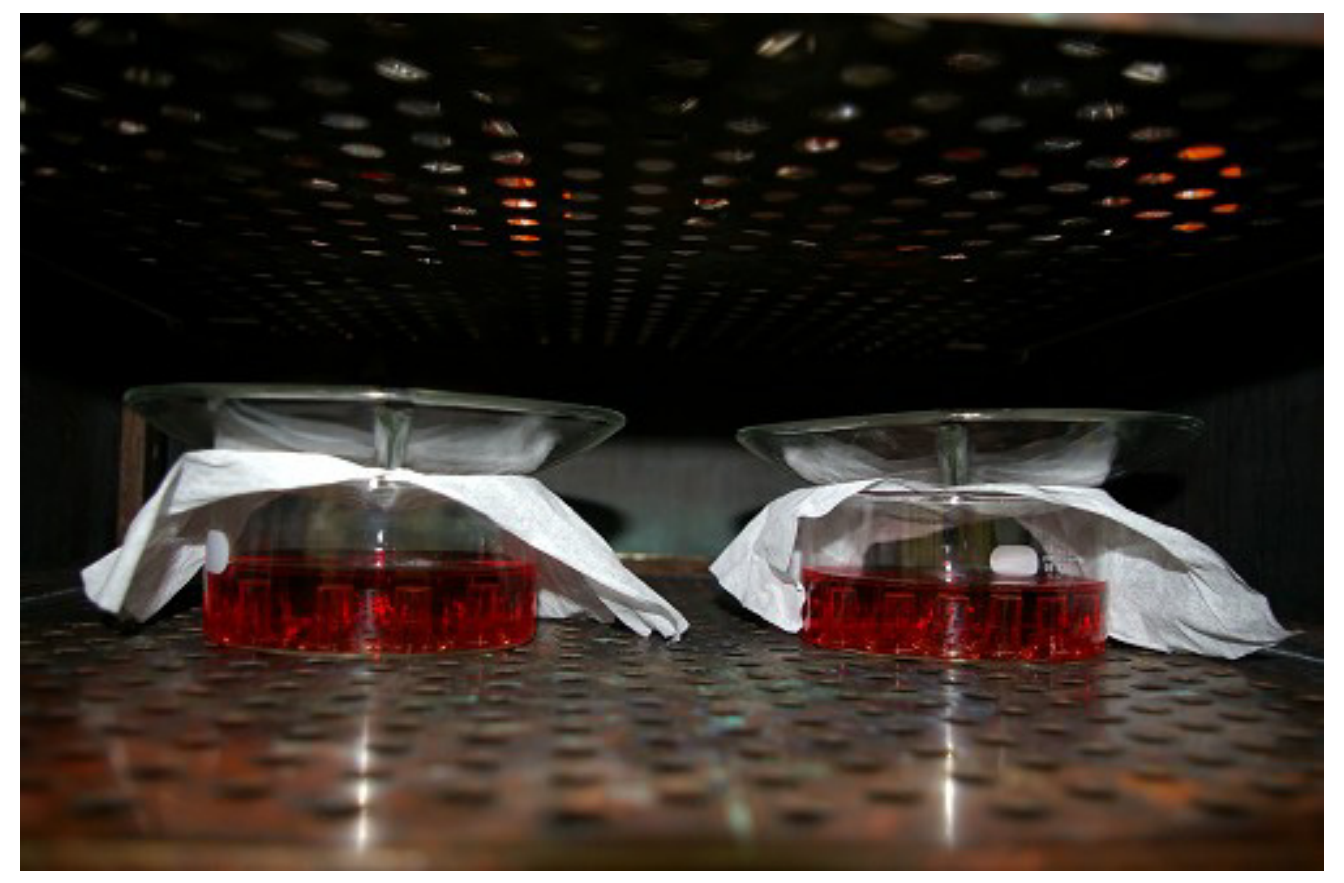

Figure 16 Astrocytes seeded on the inverted inserts in the incubator

\subsection{Astrocytes and Endothelial Cells Morphologies}

Both astrocytes and endothelial cells started to attach and spread in the cell culture vessel bottom within 12 hours of plating. Both cells were observed to attach and proliferate well under routine maintenance. As described in previous chapters, astrocytes and endothelial cells have distinct morphologies. 2 days after the cells were seeded; pictures from an optical microscope of both cells in culture were taken by a camera. Figure 17 shows the morphologies of astrocytes and ECs growing on tissue culture well plates and COL Transwell inserts, respectively. All the pictures shown in this figure were taken using a digital single lens reflex camera (Pentax K100D at 35mm focal length with aperture of 1:4) directly from the eye-piece of an inverted microscope. 

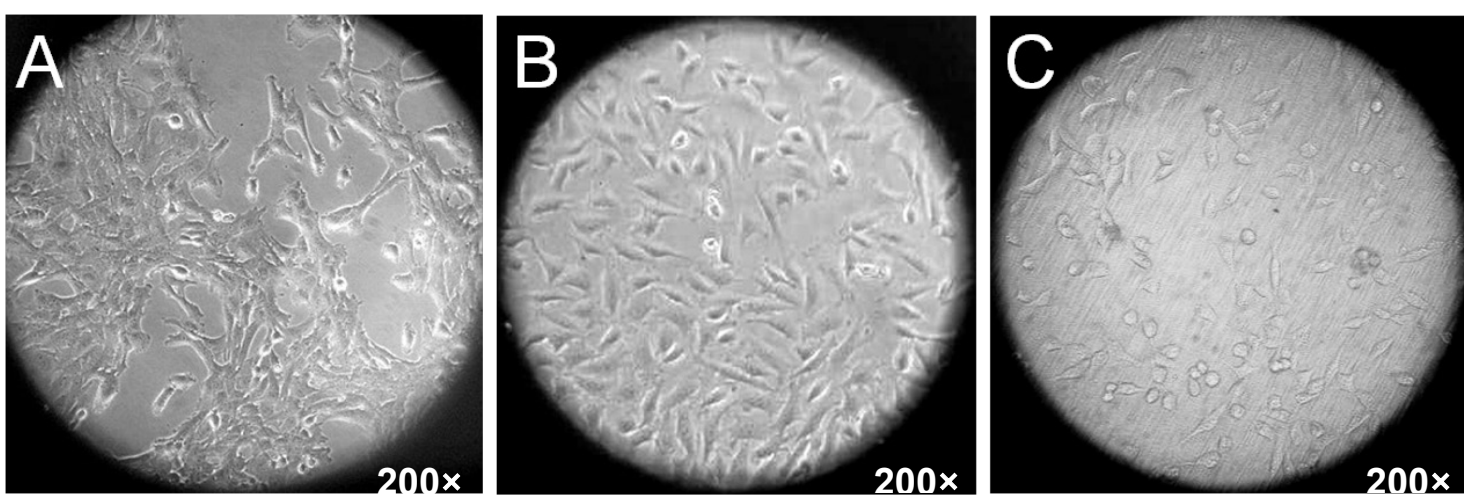

Figure 17 Cell morphologies observed from optical microscope under magnification of $\times \mathbf{2 0 0}$

Astrocytes' star like shape is obvious from Figure 2(A). The author also noticed the astrocytes grew faster than the endothelial cells and were more easily to aggregate. Figure 2(B) shows endothelial cells spreading on the plastic well bottom. The typical endothelial squamous shapes are also easy to distinguish from the picture. Endothelial cells attached well on the collagen coated filter surface of COL Transwell inserts. The structure of the collagen coated fibrous PTFE insert membrane could be identified under the microscope in Figure 2(C). There was no obvious morphological difference between the ECs growing on normal culture vessel and ECs growing on the COL Transwell inserts.

\subsection{Summary}

The chapter focuses on the engineering of the in vitro co-culture samples. After reviewing several currently available approaches attempted by other researchers, the author went to with the explaining of her own design of the sample setups. Different sample setups were introduced and compared by both schematic drawings and real life photographs. As part of the novel approach in this dissertation, the theoretical basis of 
aECM and how it was produced was revealed in this chapter. SEM images compared the difference between a normal confluent astrocytes layer and the aECM. The morphologies of the chosen cell lines were looked at in the end of the chapter too. 


\section{CHAPTER VI TEER MEASUREMENTS}

\subsection{Introduction}

As a convenient and reliable, yet non-destructive method, trans-endothelial electric resistance (TEER) measurements have been employed by various researchers to evaluate and monitor the growth of in vitro endothelial cell cultures, which in this particular case, is to evaluate the in vitro endothelial tight junctions.

The EVOM endothelial voltohmmeter from World Precision Instruments, Inc. (WPI, Sarasota, FL) was the first instrument designed specially to make routine TEER measurements in cell culture research. As a standard accessory, the EVOM comes with a set of STX2 electrodes ("chopsticks") to directly measure TEER values from the inserts in the well plates. The two electrodes are of different lengths. When they are dipped into the cell culture well, the shorter electrode goes into the insert upper chamber while the longer one stays outside of the insert in the lower chamber of the well. During the TEER measurements, the electrodes should not touch the bottom of either the insert or the well.

Figure 18 shows the EVOM and STX2 electrodes in real life.

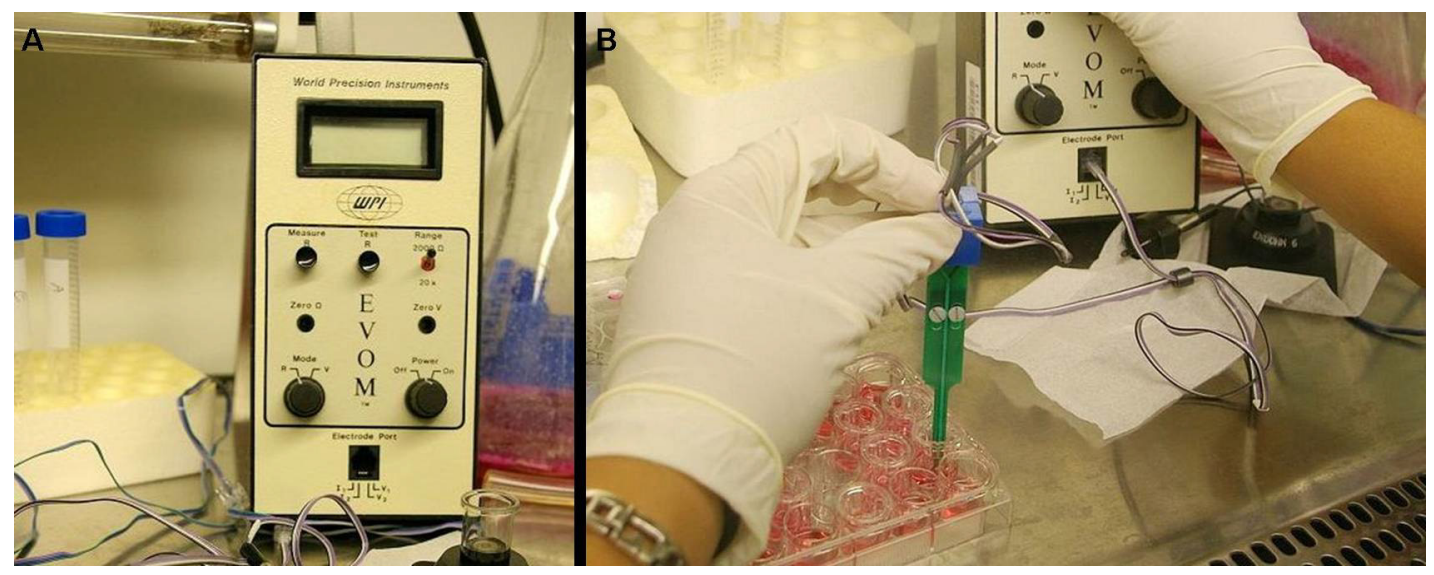

Figure 18 TEER measurement instrument. A: EVOM Endothelial Voltohmmeter.

B: STX2 electrodes measuring TEER value of the insert in the well plate 


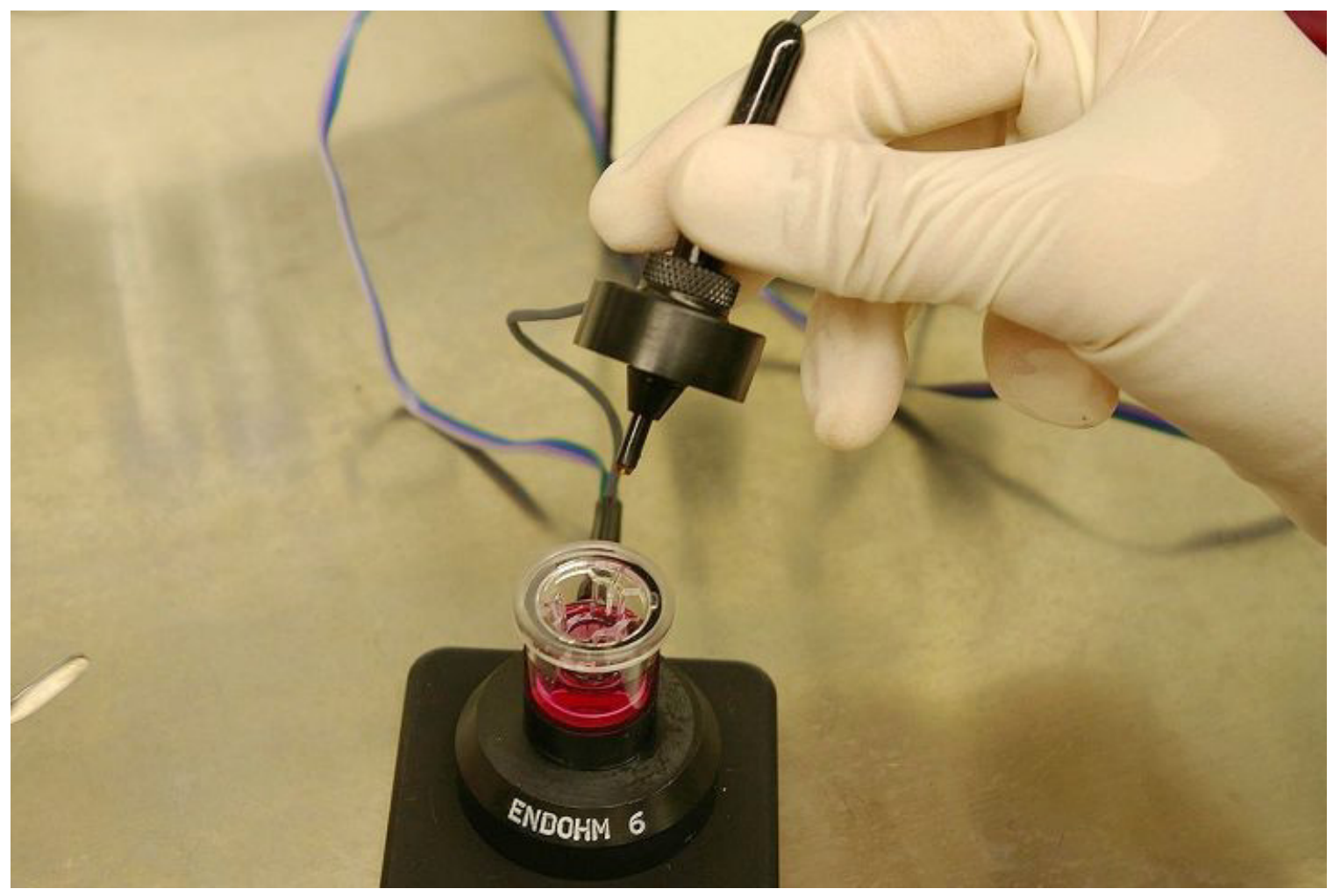

Figure 19 Endohm TEER measurement chamber for $6.5 \mathrm{~mm}$ diameter Transwell

\section{inserts}

Endohm tissue resistance measurement chamber is another type of TEER measurement tool designed to be used together with EVOM for various insert types and more accurate quantitative measurements. Figure 19 is a demonstration of an Endohm chamber designed for $6.5 \mathrm{~mm}$ diameter Transwell inserts which suit the 24-well plates. This Endohm chamber has the exact size of the well from the 24-well plates. The chamber and its cap each contain a pair of concentric electrodes, respectively. When taking TEER measurements, the Endohm should be filled with media whose volume is equal to that from the normal cell culture condition. The media should be the same culture media but without serum in order to avoid the proteins from the serum to aggregate on the electrodes and affect the measurement accuracy. The height of the cap 
electrode can stay fixed for a chosen insert which eliminates the inconsistency between measurements. What's more, Endohm's apposing circular electrodes which symmetrically situated beneath and above the membrane allow a more uniform current density to flow across the membrane than with STX2 electrodes. According to WPI, the background noise from the resistance of a blank insert is reduced from $150 \Omega$ (as with the STX2 electrode) to less than $5 \Omega$ for Endohm chambers.

\subsection{Methods}

In the earlier trials of the experiment, the STX2 electrodes were employed in the TEER measurement because it came with the EVOM device. However, the "chopsticks" showed unpredictable inconsistency in the readings, which were easily affected by the vertical position of the electrodes or the media levels in the cell culture chambers. Thus after the initial 3 trials, Endohm was purchased to do the rest of the TEER measurements.

For all the samples setups, the number of days were counted starting from the endothelial cells were counted. For examples, for the "CO" and "CO+aECM" samples, the day when the endothelial cells were seeded on the apical sides of the inserts was defined as Day- 0 . The endothelial cells were untouched on Day-1 so that they could recover from cryo-preservation or sub-culturing dislodging shock. Starting from Day-2, the TEER values were measured daily for the membranes on the inserts using Endohm chambers connected with EVOM. Since cells on the apical sides of the inserts from other samples setups such as "EC" and "Pseudo" were seeded on the same day when the endothelial cells for the $\mathrm{CO}$ and $\mathrm{CO}+\mathrm{aECM}$ samples were seeded, the TEER measurements could thus be synchronized. 
Starting from Day-2, TEER measurements were taken for all the sample insert membranes. First of all, the culture media was removed from both upper and lower chambers of the samples. The upper and lower chamber of the sample wells containing inserts were filled with $100 \mu 1$ and $600 \mu 1$ warm serum-free DMEM (Gibco), respectively. $600 \mu 1$ of the warm serum-free DMEM was added to the Endohm chamber too. Then the each insert was picked up carefully by a pair of sterile tweezers and placed into the Endohm chamber to be measured by the EVOM. After the measurements, the inserts were placed back into their corresponding wells. The serum-free DMEM was replaced by normal culture media again and the samples were put back into the incubator.

During the measurement, two readings were taken for each insert and the original results were expressed as the average values of the readings. The author found the differences between the two readings were usually \pm 1 . In the initial several trials, the TEER for a blank insert was always measured alongside the samples. As the Endohm manufactory stated, the TEER reading for the blank insert was always around 5 throughout the experiments. Starting from Trial \#4, the adjusted TEER measurement results were recorded as the actual readings minus 5 . The final results were all adjusted based on the aforementioned conditions and expressed in $\Omega \mathrm{cm}^{2}$, which were calculated by multiplying the adjusted readings by the effective surface area $\left(0.33 \mathrm{~cm}^{2}\right.$ for Transwell inserts for 24-well plates) of the filter membrane.

\subsection{Results and Discussions}

A total of 13 trials were performed in the dissertation work. Non-collagen coated inserts were employed in the study from Trial \#1 through Trial \#7. The COL inserts were used starting from Trial \#2. The results for both types of inserts were presented in the 
dissertation annual report. However, both the committee members and the author were convinced that due to the poor cell attachment of the non-collagen coated inserts, these inserts were not suitable for the specific in vitro study and the corresponding results should not be included in the final dissertation discussion. For this reason, only the TEER values from COL samples of are presented in the following graphs starting from Trial \#2. The values were expressed in average \pm standard deviation (4 replications per each trial).

As shown from the graphs, in the initial stage of the dissertation the cell cultures were maintained for 8 or 9 days depending on the lab schedules and available resources. Because of unexpected contaminations, Trial \#4 was terminated before any analysis or data acquisition.

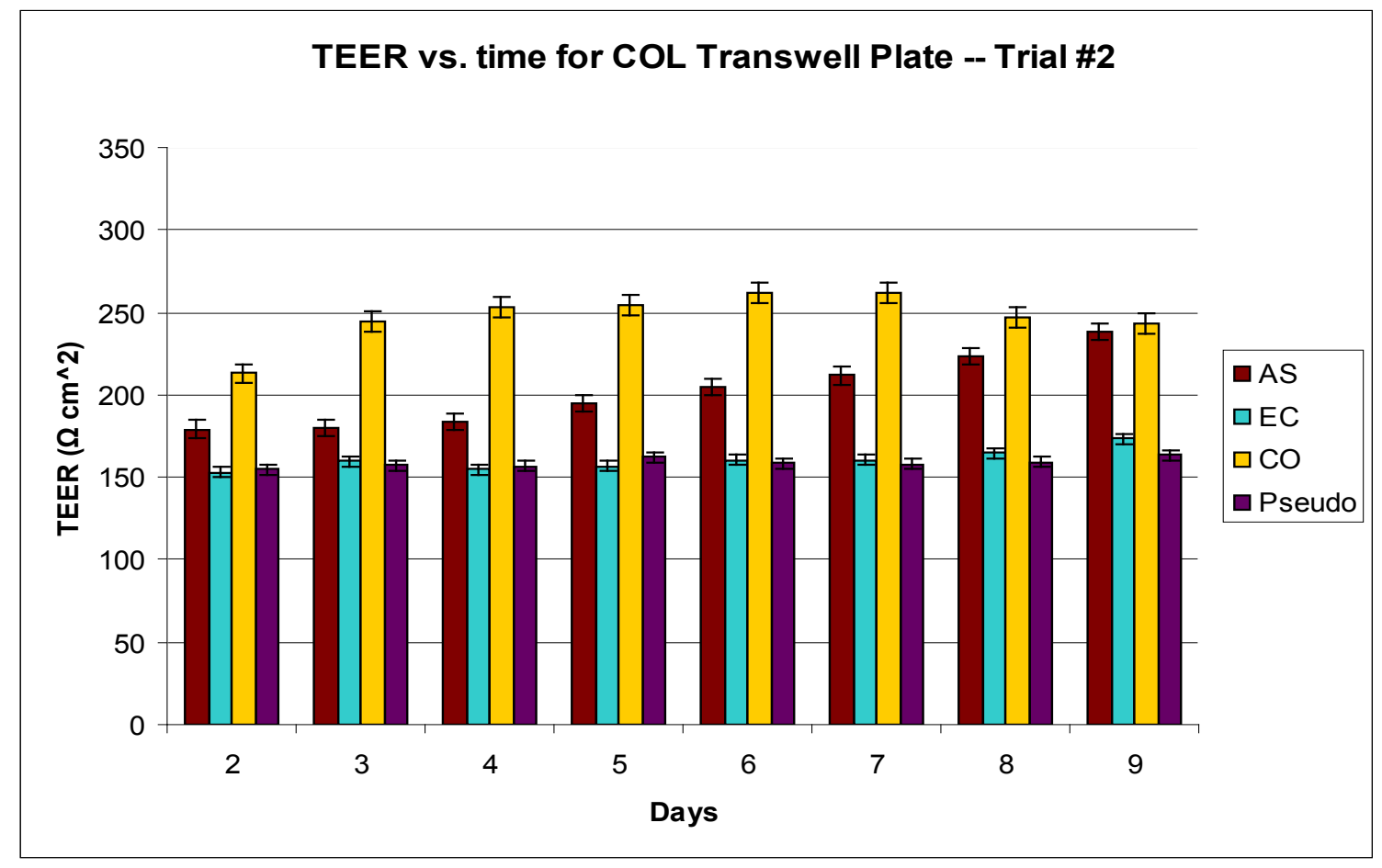

Figure 20 TEER measurement results for Trial \#2 


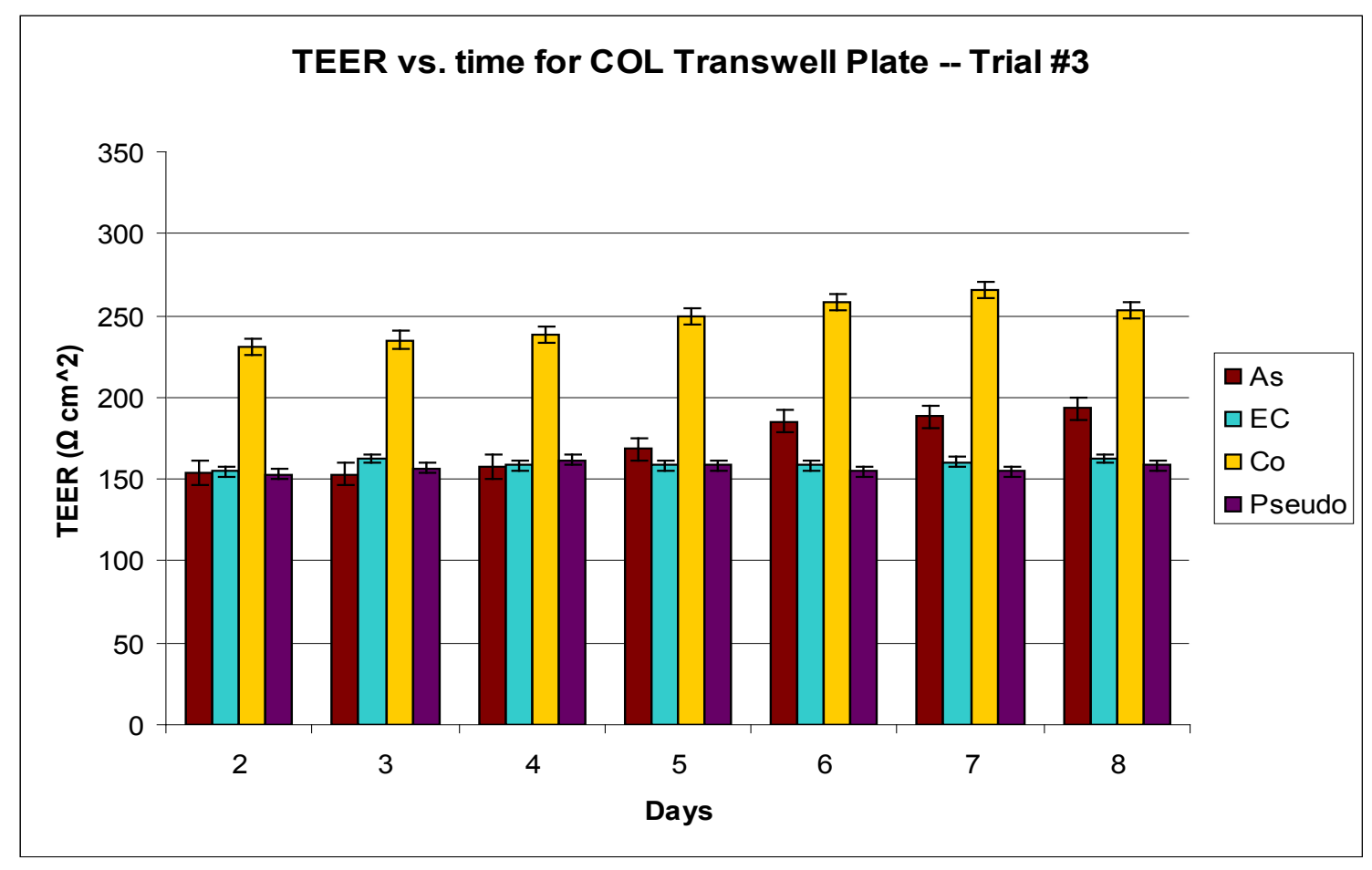

Figure 21 TEER measurement results for Trial \#3

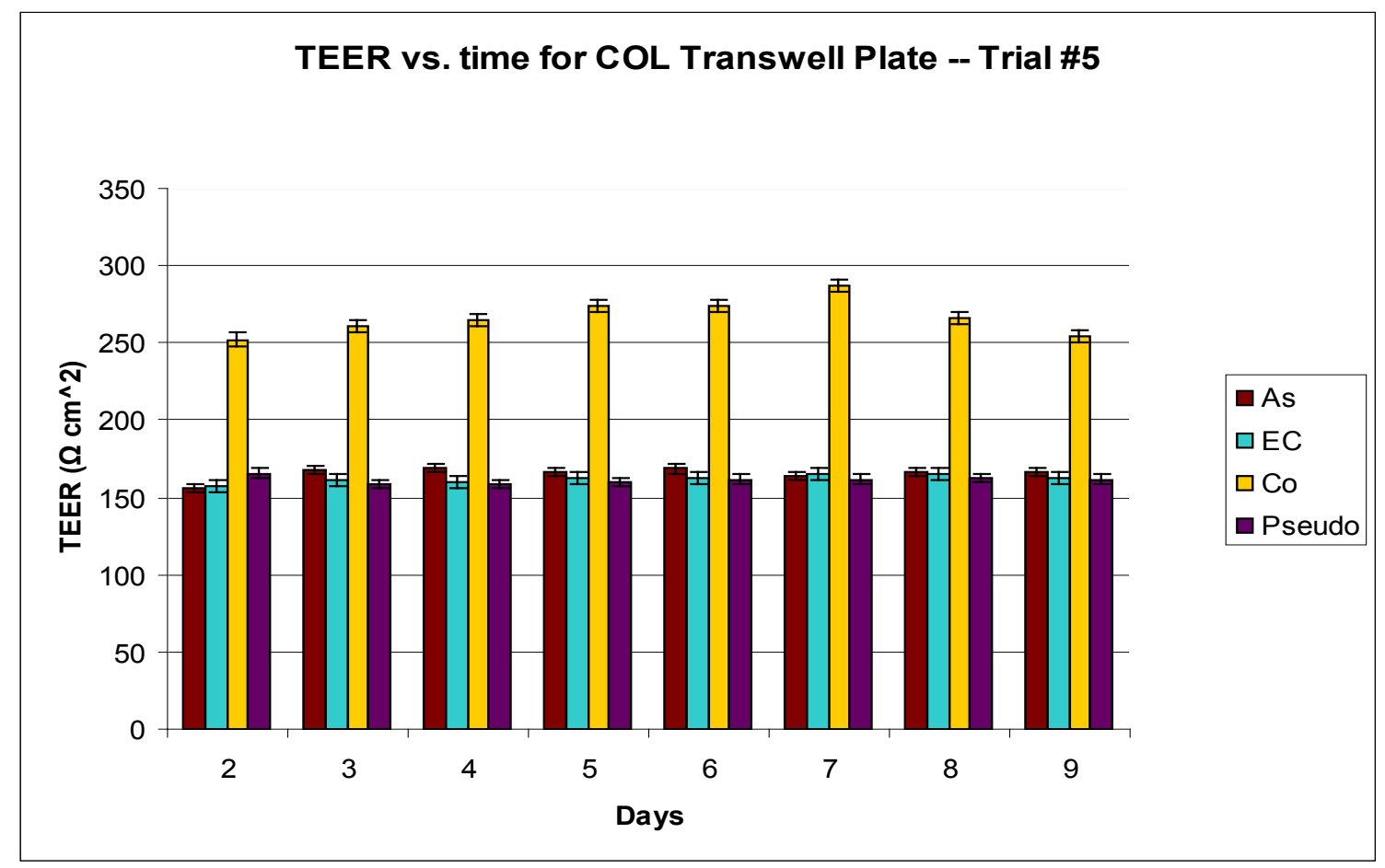

Figure 22 TEER measurement results for Trial \#5 


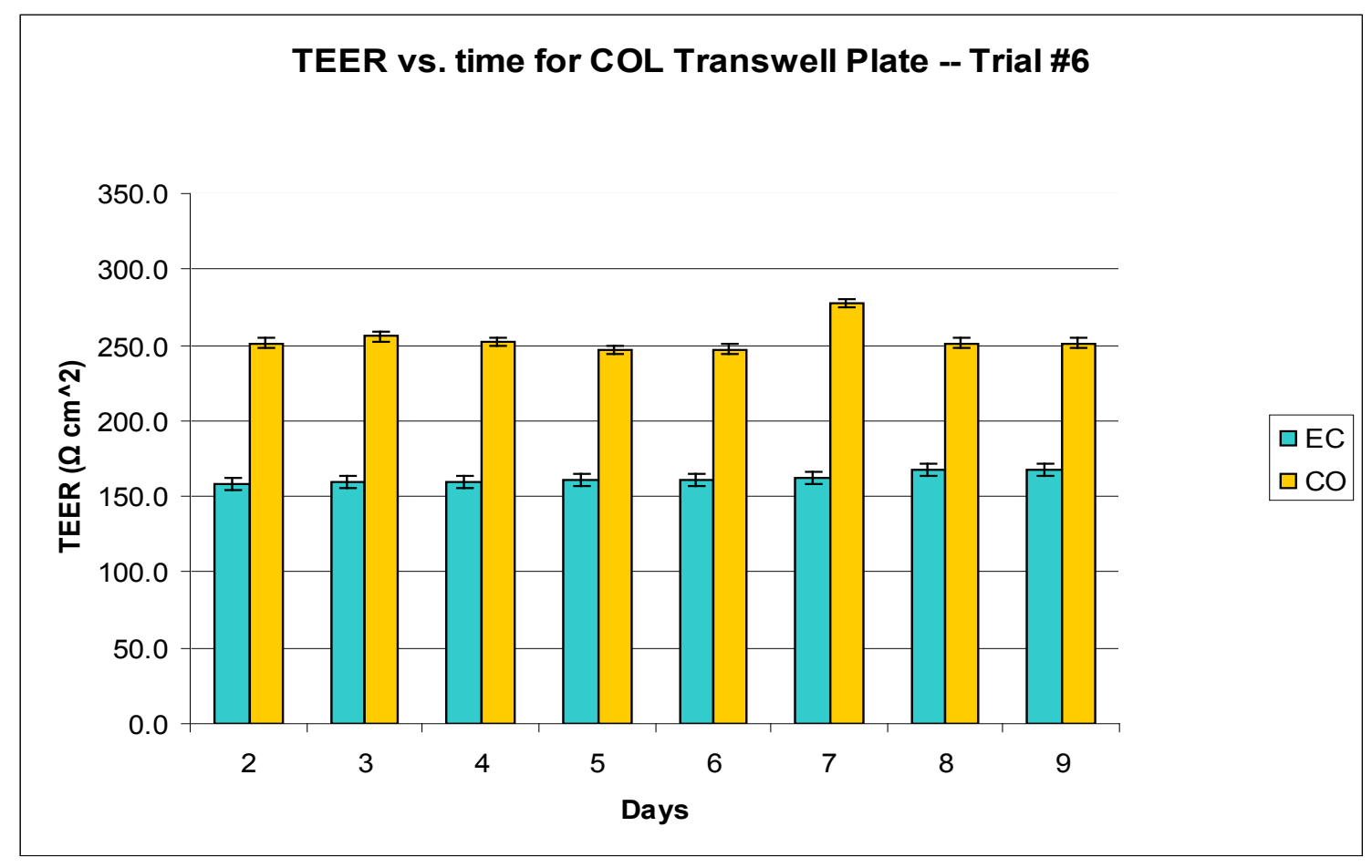

Figure 23 TEER measurement results for Trial \#6

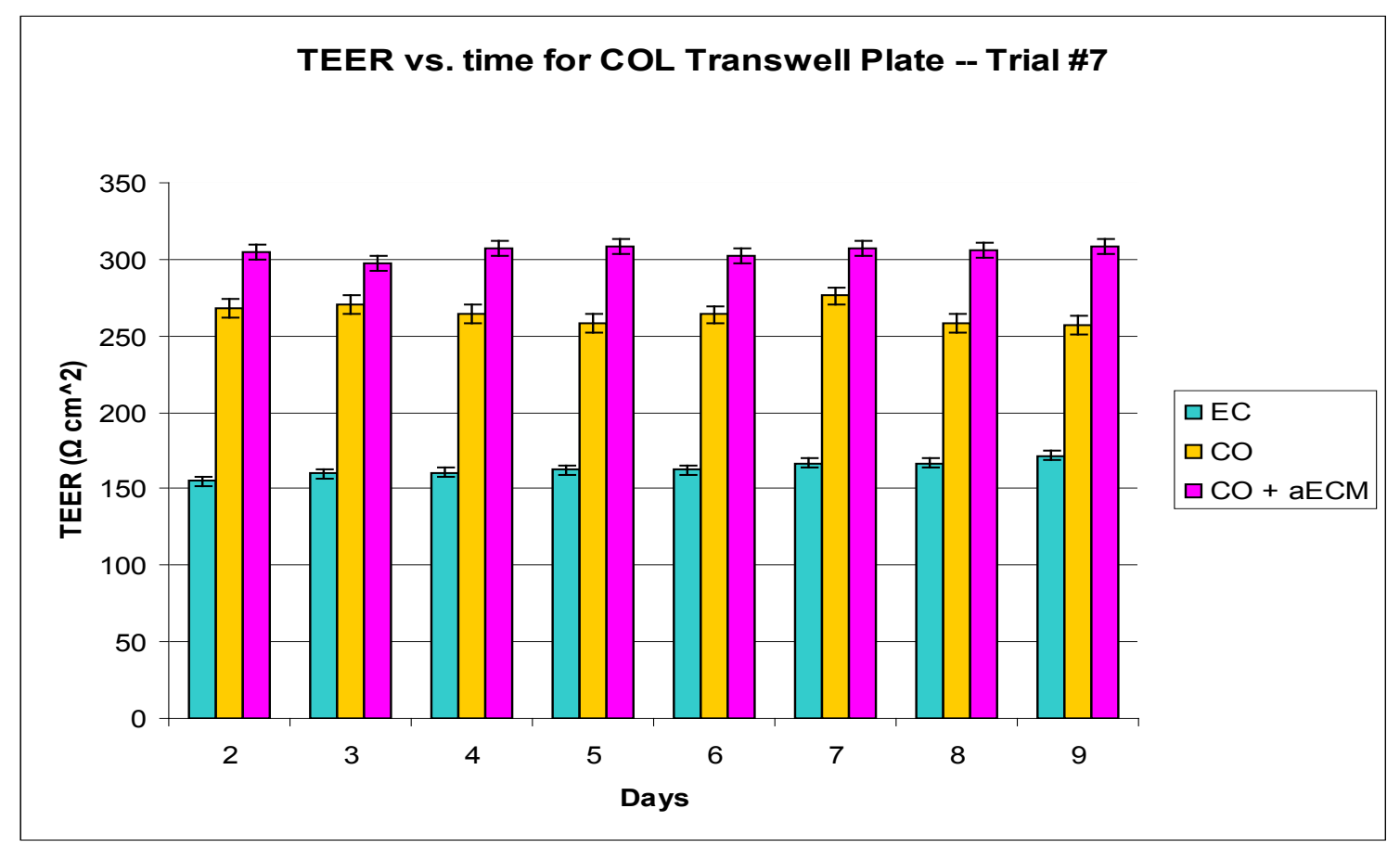

Figure 24 TEER measurement results for Trial \#7 


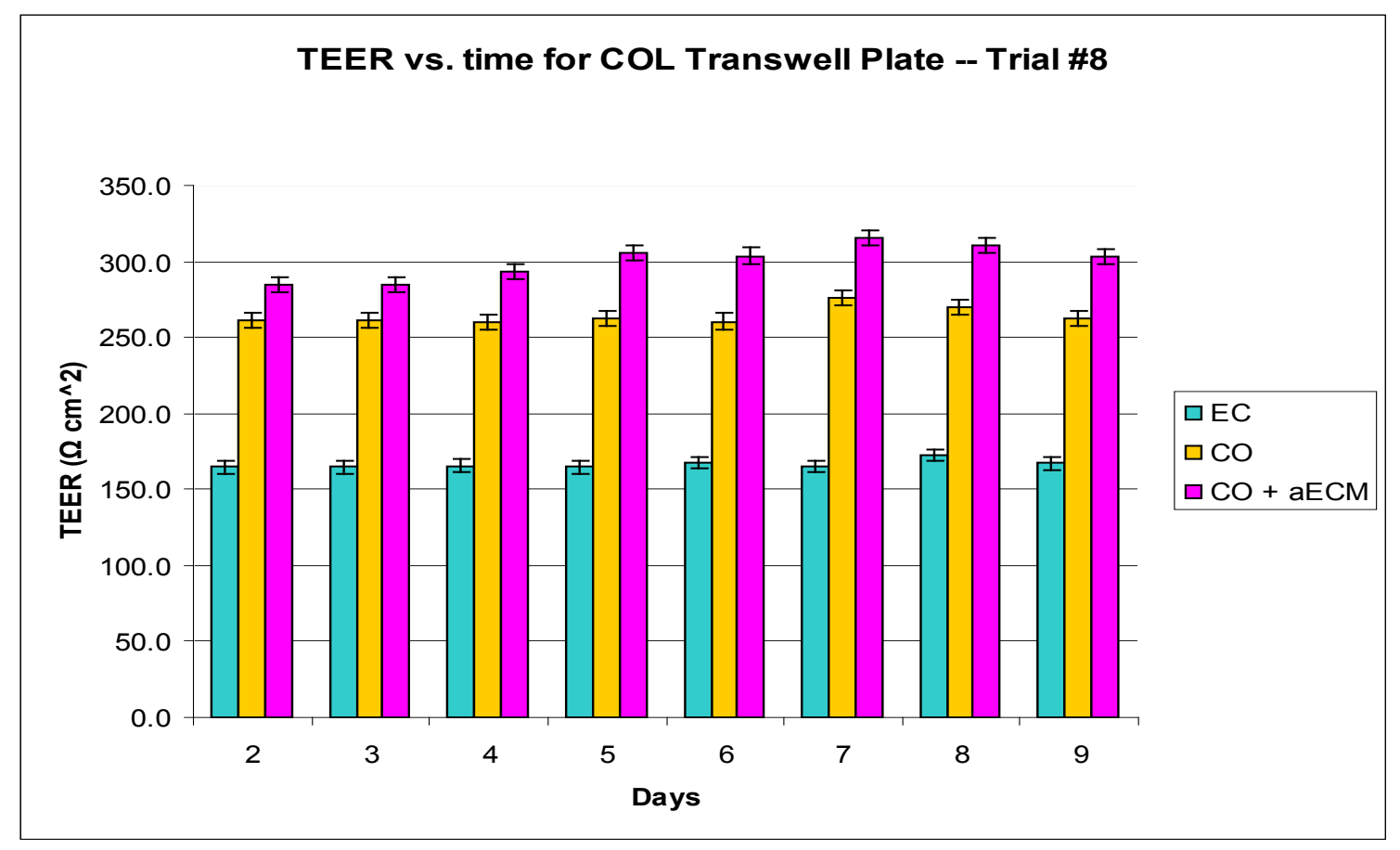

Figure 25 TEER measurement results for Trial \#8

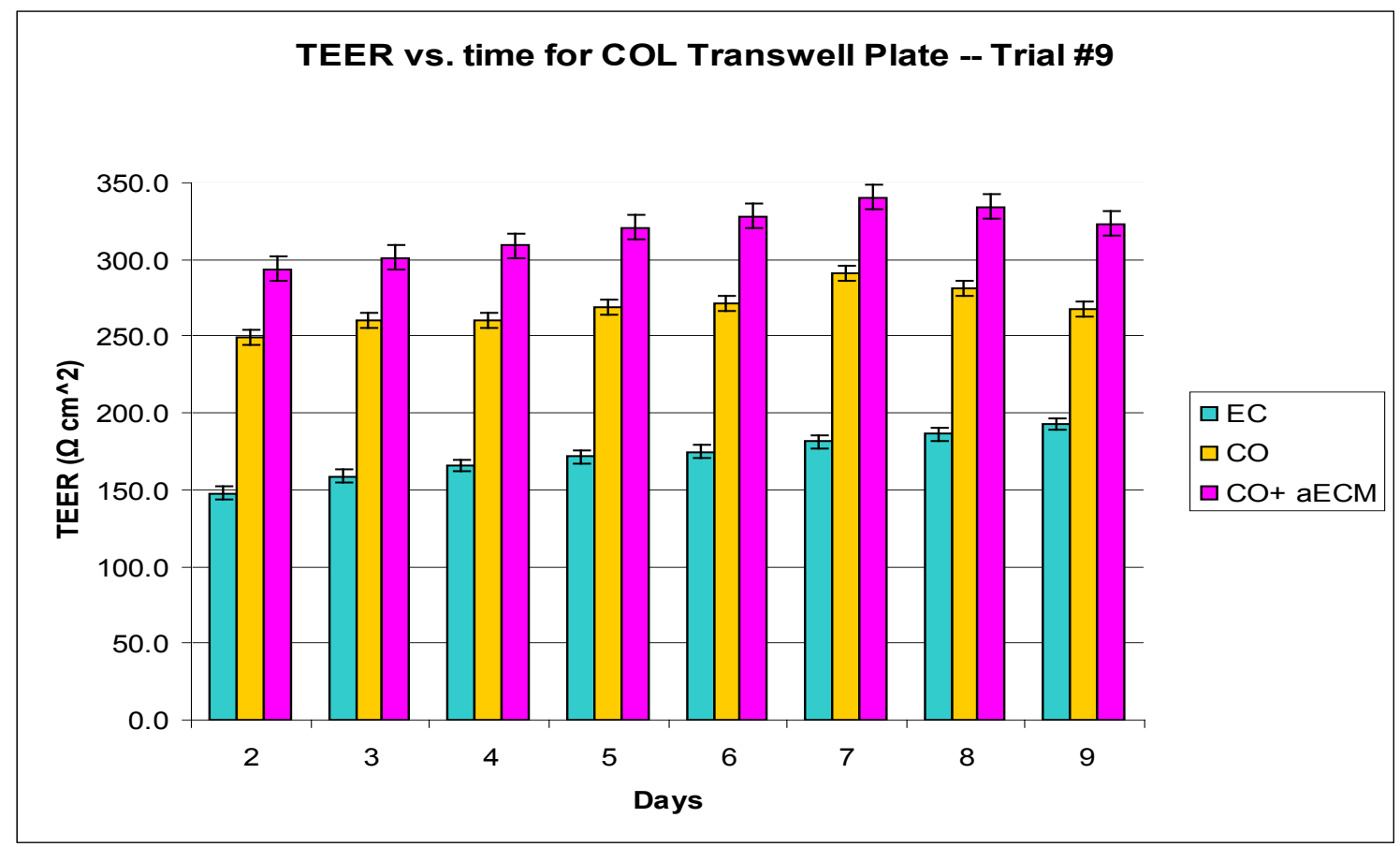

Figure 26 TEER measurement results for Trial \#9 


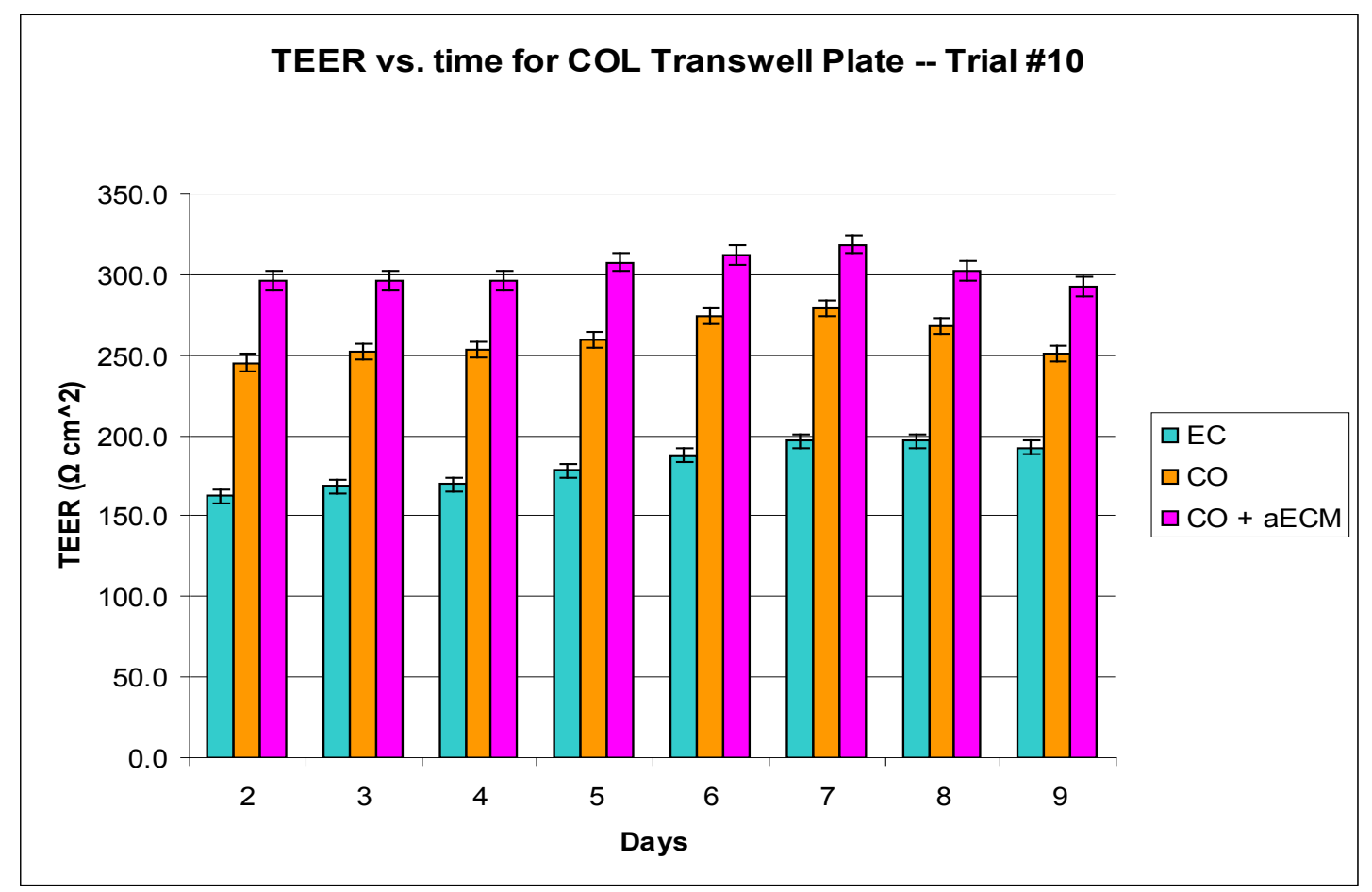

Figure 27 TEER measurement results for Trial \#10

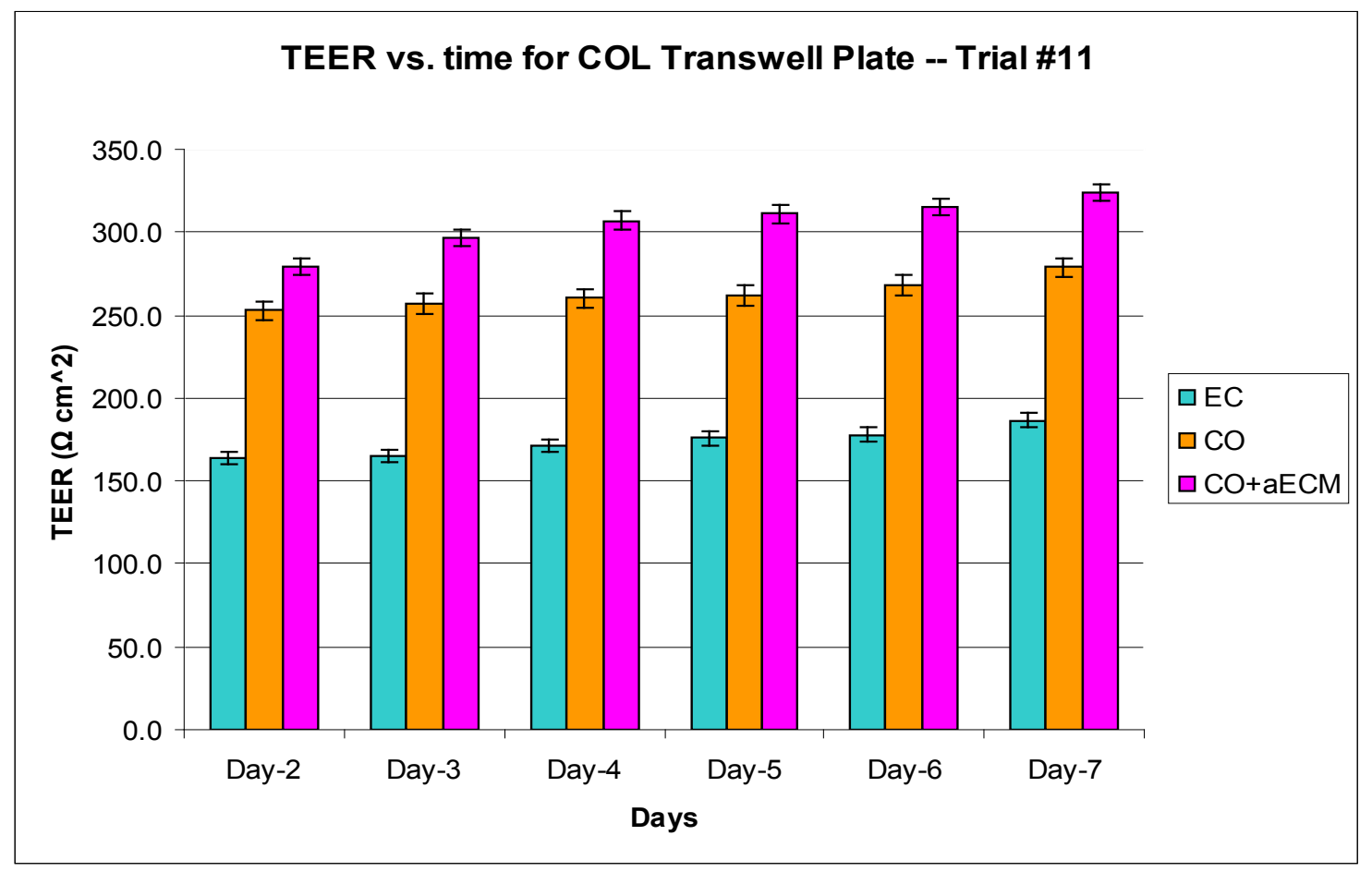

Figure 28 TEER measurement results for Trial \#11 


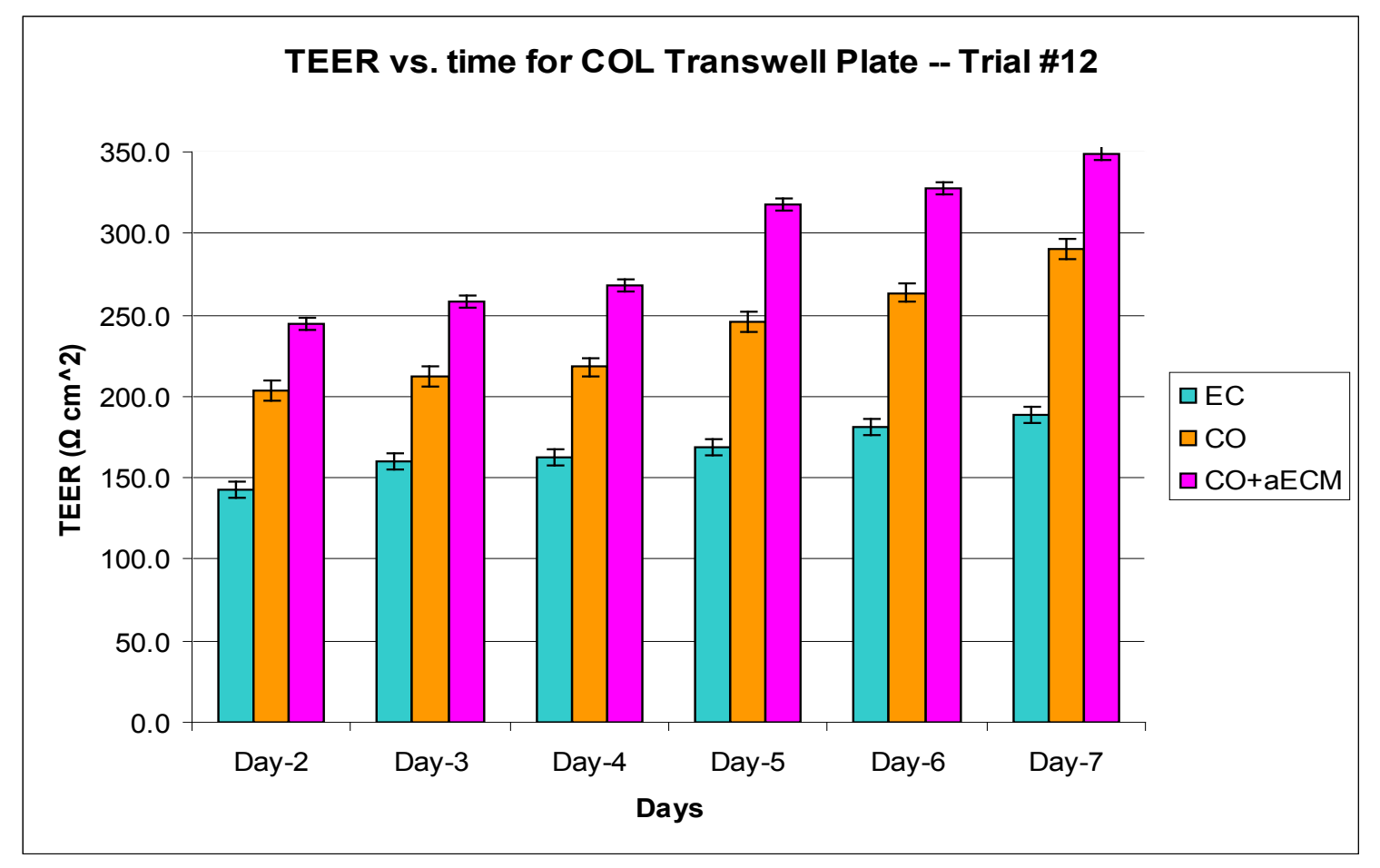

Figure 29 TEER measurement results for Trial \#12

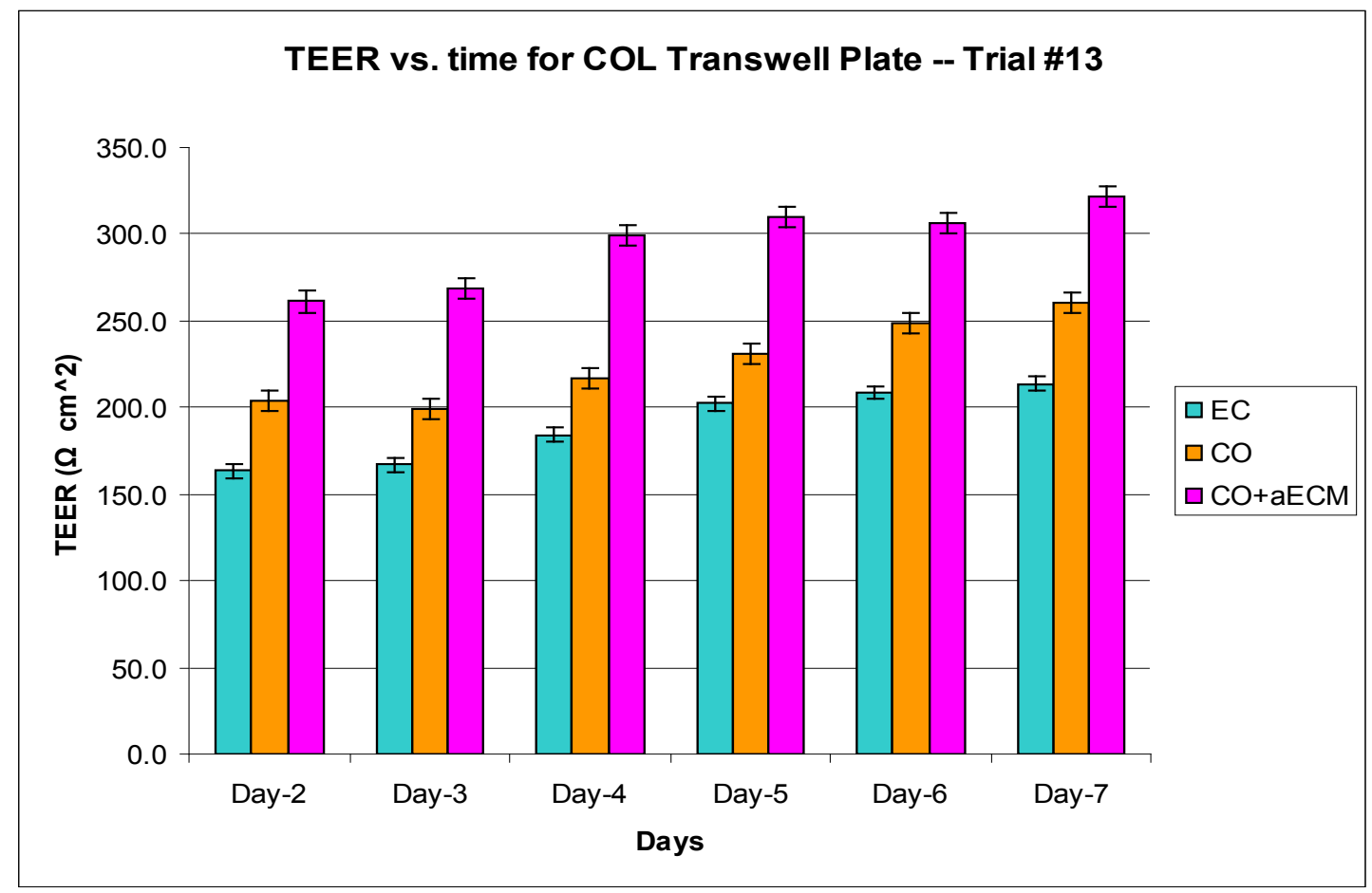

Figure 30 TEER measurement results for Trial \#13 
The trend of the results from the earlier trials (Trial \#2 through Trial \#6) showed that the TEER values for "CO" samples started at around or just below $250 \Omega \mathrm{cm}^{2}$ and gradually raised with the highest being $286.6 \pm 3.0 \Omega \mathrm{cm}^{2}$ in Trial \#5 on Day-7. The TEER values for the "CO" samples were testified to be statistically significant higher than those of the other samples by one-way ANOVA analysis $(\alpha=0.05)$. The TEER values were lower than the primary BBEC culture by Garberg et al. which were 500-800 $\Omega \mathrm{cm}^{2}$ (7), but significantly higher than the ECV304 cell line $\left(100 \Omega \mathrm{cm}^{2}\right)$, which has been used for feasible immortalized BBB models by several groups, and found to have relative high correlation between in vivo and in vitro permeability studies $(44,87,88)$. As in vitro models, the TEER values were not expected to be as high as those found in in vivo environment, or even as high as the aforementioned primary cell cultures. The results from these earlier trials fall between the range of established primary culture and relatively successful immortalized BBB models, the author felt the study could continue based on the current sample setups with necessary improvement of experiment techniques. The repeatability should be an important aspect to evaluate in the assessment. In the earlier trials, the author was still trying to figure out the optimal method to produce the aECMs, especially in the lysis procedure. While at the same time "Pseudo" setups were attempted by the authors following examples by other researchers. However, the author found out that the TEER values were not significantly different between "Pseudo" and "EC" samples by student t-test analysis $(\alpha=0.05)$ in a day-to-day comparison throughout the initial 3 trials. In the "Pseudo" case even though astrocytes presented remotely in the same chamber, but their secretions may not reach the high enough concentration so as to affect the junctional formation between the endothelial 
cells. Given limited funding and materials, the author decided not to continue with the "Pseudo" setup. The TEER values for "AS" and "EC" samples were not significantly different either. Since this research was to examine electrical resistance between endothelial cells, the TEER values of the astrocytes layers were no longer displayed in the following trials.

By the beginning of Trial \#7, the author had mastered the effective method to produce aECMs with consistent quality so the aECMs were included in the co-culture sample building starting from Trial \#7. Trials \#7 through \#10 demonstrated the TEER values for both " $\mathrm{CO}$ " and "CO+aECM" samples were significantly higher than the "EC" samples. The "CO+aECM" samples showed significantly higher TEER values than the "CO" samples. Statistical study were carried out by one-way ANOVA analysis $(\alpha=$ 0.05). However, since there were no literature "CO+aECM" TEER value data to compare with the "CO+aECM" data. Additional studies including transport study and immunochemistry studies needed to further evaluate the endothelial tight junction in all the samples.

Similar as the earlier trials, the TEER values for the "EC" samples in Trials \#7 through \#10 were maintained at a relatively stable level while the values slightly increased over the culture period. The TEER values for the "CO" and "CO+aECM" samples were more established starting from Day-4 which continued to increase until Day-7. However, the TEER values for these co-culture models were seen to slowly decrease from Day- 8 for the majority of the samples. Thus Day-7 was selected as the optimal day for transport studies which will be introduced in the next chapter. Trials \#11 through \#13 were dedicated in solute transport study and their culture period were 
terminated on Day-7. In these later trials, the "CO+aECM" samples still showed higher TEER values and those of the "CO" samples and both co-culture samples' TEER values were significantly higher than the "EC" samples.

For all the trials, the TEER values for the co-culture samples were consistent. One-way ANOVA analysis showed that "EC", "CO" and "CO+aECM" samples were significantly different from each other on a daily basis: the TEER values for both "CO" and "CO+aECM" samples were significantly higher than the "EC" samples for all the trials by one-way ANOVA analysis when $\alpha=0.05\left(\mathrm{p}=2.06 \times 10^{-12}\right.$ on Day-7). The TEER values for "CO+aECM" samples were also significantly higher than the "CO" samples by one-way ANOVA analysis when $\alpha=0.05\left(\mathrm{p}=3.7810^{-5}\right.$ on Day-7). The $\mathrm{p}$-value for the significance between "CO" and "EC" samples was $3.31 \times 10^{-8}$ on Day-7.

\subsection{Summary}

This chapter examined the endothelial tight junction formation by evaluating the sample membranes' electrical resistance through a method call TEER measurement. "EC", "Pseudo", "CO" and "CO+aECM" were among the test candidate. "AS" samples' TEER values were also taken in the earlier trials, and they showed similar readings as those of the "EC" and "Pseudo" samples.

The TEER values for "EC" "CO" and "CO+aECM" samples were found to be consistent between different trails. "CO" and "CO+aECM" samples' TEER values were both significantly higher than those of the "EC" samples, and at the same time "CO+aECM" samples demonstrated significantly higher TEER values than the "CO" samples. The values for both of the co-culture samples were comparable to some currently available in vitro BBB model attempts and these samples were approved to be 
relevant setups for BBB in vitro modeling. The TEER values for these samples gradually establish through Day-7 and started to decrease from Day-8. "EC" "CO" and "CO+aECM" samples from Trials \#11 through \#13 were engaged in solute transport study in the next chapter. 


\section{CHAPTER VII TRANSPORT STUDIES}

\subsection{Introduction}

As was introduced in previous chapters, the BBB keeps out almost all nonlipophilic molecules larger than 500 Dalton from entering the brain blood stream freely through passive diffusion. In this chapter, several different compounds were proposed to test the permeability of the in vitro samples over the time.

Table 4 Proposed test compounds in the BBB transport studies

\begin{tabular}{|l|l|l|l|}
\hline Compound Name & Molecular & UV-Vis & Solubility \\
\hline Calcein & 622.55 & $490 \mathrm{~nm}$ & Hydrophilic \\
\hline Caffeine & 80.70 & $273 \mathrm{~nm} \& 232 \mathrm{~nm}$ & Hydrophilic; \\
\hline Doxorubicin & 579.98 & $245 \mathrm{~nm}$ & Soluble in chloroform \\
\hline Keppra ${ }^{\circledR}$ & & & Hydrophobic \\
\hline (levetiracetam) & 170.21 & $244 \mathrm{~nm}$ & Hydrophilic; \\
\hline Vincristine sulfate & 923.04 & N/A & Soluble in: chloroform, \\
\hline Sicotine & & & ethanol \& acetonitrile \\
\hline
\end{tabular}


A list of test compounds was proposed in the beginning of the dissertation work and is listed in Table 4. The different compounds were selected based on their different sizes, solubility and functionalities. For example, sucrose has been a popular candidate among researchers in evaluating BBB permeability. DOX and Keppra ${ }^{\circledR}$ were selected to be the drug candidates. DOX is a chemotherapy agent and has been reported as a known substrate of p-glycoprotein which actively repels this drug out of the brain. Keppra ${ }^{\circledR}$ is an anti-epilepsy agent which is claimed to be able to pass the BBB by its manufactory (UCB Pharmaceuticals, Inc.). Vincristine, another chemotherapy agent with the molecular weight of more than 900 Dalton was also selected as a drug candidate in the experiment. Caffeine and nicotine was selected because their small molecular size and their possibility to pass through the BBB. Calcein is a molecule that has not been reported to affect the brain, and its molecular size is around the BBB molecular weight threshold and it would be interesting to see how the in vitro samples would react to it.

Because of the on campus facility restraints, the author was not able to conduct radio labeled transport study which is the most popular approach in this field. Instead, an optical spectrophotometer was employed in determining the amount of solute in the experiments. Spectrum scans at the wavelength of $200 \mathrm{~nm}-800 \mathrm{~nm}$ were preformed on all the proposed compounds to determine their absorbance peak, respectively. The absorbance listed in the above table all came from such experiments.

\subsection{Methods}

In vitro samples from several later trials were selected for the transport studies. As indicated in the previous chapter, the TEER values reached their peaks on Day-7 and started to slowly lessen over the next couple of days. Hence Day- 7 was decided as the 
day for the transport studies for optimal results. Trials \#11 through \#13 were dedicated for the transport studies. Since each setup had four replications in each trial, one or two of the replications would be dedicated for one type of test compound in the transport study. One blank insert for each different compound would be used as the control to undergo same test procedures. The test compounds were pre-dissolved in Phenol red-free DMEM (Invitrogen, Carlsbad, CA). The stock concentration of the test compound solutions were empirically decided to be $50 \mu \mathrm{g} / \mathrm{ml}$, which was the optimal concentration for the working range of the Cary 100 UV-Vis spectrophotometer (Varian, Palo Alto, CA). Silica spectrophotometer cuvettes and cuvette washer/drier (Sigma-Aldrich) was employed in the experiments for more accurate and consistent readings. Figure 31 shows the spectrophotometer and cuvette images from their manufactories, respectively.

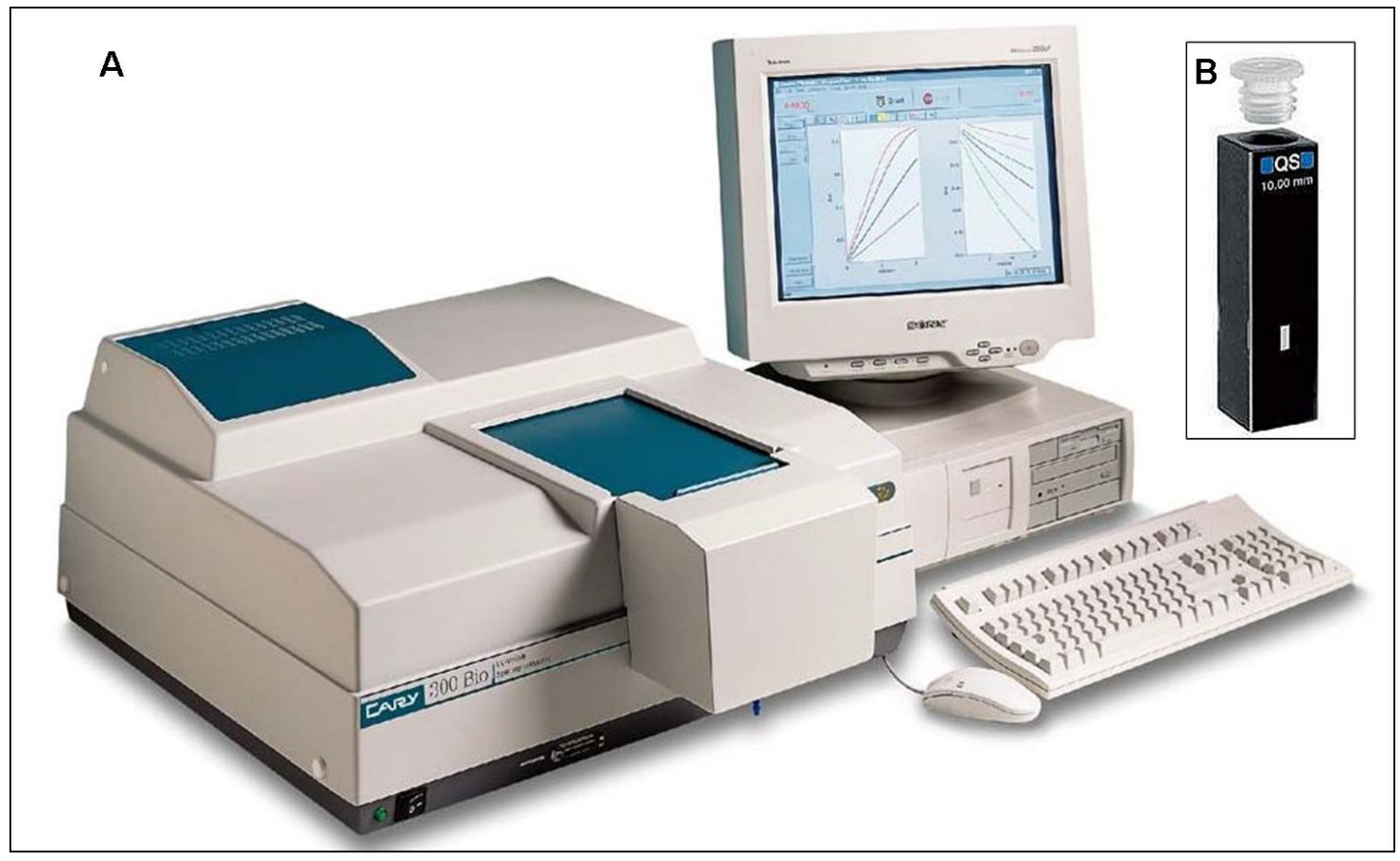

Figure 31 Measurement instruments in the transport studies. A: Cary $100 \mathrm{UV}-\mathrm{V}$ is spectrophotometer. B: Silica spectrophotometer cuvette 
At the end of the 7-day culturing period, growth media were removed from selected samples following by gentle rinsing with warm phenol red free pure DMEM. Then $200 \mu$ of the stock concentration of compound solutions were added to the upper chambers of each insert. The lower chambers of each insert were supplemented with $1220 \mu \mathrm{l}$ of phenol-red free pure DMEM, respectively. The media levels inside and outside of the insert were thus kept leveled to avoid any hydraulic force influence. The sample plate was incubated at normal conditions with $5 \% \mathrm{CO}_{2}$ and at $37^{\circ} \mathrm{C}$ except during sample extraction. At predetermined times $(15,30,45,60,90$ and $120 \mathrm{~min}) ; 110 \mu \mathrm{l}$ of sample was withdrawn from the lower chamber of each sample, respectively. $21 \mu$ of compensation volume of the solution was removed from the upper chamber of the corresponding sample to maintain the media volume balance between two chambers. The $110 \mu 1$ extracted samples were kept in sterile capped $1 \mathrm{ml}$ centrifuge vials (Fisher Scientific) for later measurement. Because of the small amount of the sample size, the measurement should be taken immediately following the sample extraction to keep the error from sample evaporation to the lowest level.

Optical absorbance of each sample was determined by UV absorbance at their unique peaks using the Cary-100 spectrophotometer. In the initial several trials, the readings were taken twice for each sample in order to see if there was any variance in the readings. It was found out that for the same sample, when scanned under the same condition in adjacent times, the reading were always uniform. However the silica cuvette needed to be washed every time before new measurement and there was only one pair of such cuvette. Considering the small amount of the sample, it would be unrealistic to store the sample for future use. Thus one reading was taken for each sample in majority of the 
experiment and the sample was dumped right after the measurement to save time for the rest of the measurements.

Table 5 Serial dilution concentration demonstration.

\begin{tabular}{|l|l|}
\hline Dilution \# & Concentration $(\mu \mathrm{g} / \mathrm{ml})$ \\
\hline 0 & 50.0 \\
\hline 1 & 37.5 \\
\hline 2 & 28.1 \\
\hline 3 & 21.1 \\
\hline 4 & 15.8 \\
\hline 5 & 11.9 \\
\hline 6 & 8.90 \\
\hline 7 & 6.67 \\
\hline 8 & 5.00 \\
\hline 9 & 3.75 \\
\hline 10 & 2.82 \\
\hline 11 & 2.11 \\
\hline
\end{tabular}

Serial dilution calibration curves were made for each candidate compound in order to later determine the concentration of the sample solutions. For each test compound solution, starting from the stock concentration of $50 \mu \mathrm{g} / \mathrm{ml}$, a $75 \%$ gradient was made for each subsequent dilution for a total of 12 serially diluted samples. A demonstration of the concentration of serially diluted samples is displayed in Table 5. The optical absorbance of the diluted samples were read by the spectrophotometer at their 
unique peaks and plotted against the corresponding concentration. A linear curve was fitted to each plot to obtain the relationship between the absorbance and the concentration. For compounds that had saturated readings on the higher concentrations, the saturated values were discarded but a total of more than 10 readings should be taken for each compound. For compounds that had more than one absorbance peaks in the UVVis range, all the possible curves were drawn and the one with the highest $\mathrm{R}^{2}$ value was selected to be used as the absorbance - concentration calibration curve. Demonstrations of the spectrum scans and the calibration curves are displayed in Figure 32 through Figure 36. Figure 32 shows an example of calcein concentration serial dilution scans from Trial \#9 at the absorbance peak of $490 \mathrm{~nm}$, where the starting concentration was $28.125 \mu \mathrm{g} / \mathrm{ml}$, and the dilution gradient was $75 \%$. Figure 33 shows the calcein concentration serial dilution absorbance vs. concentration chart from Trial \#9 at the absorbance peak of $490 \mathrm{~nm}$. The starting concentration was $28.125 \mu \mathrm{g} / \mathrm{ml}$, and the dilution gradient was $75 \%$. $\mathrm{R}^{2}=99.30 \%$. Figure 34 shows the example of caffeine concentration serial dilution absorbance vs. concentration chart from Trial \#11. The starting concentration was $28.125 \mu \mathrm{g} / \mathrm{ml}$, and the dilution gradient was $75 \%$. Absorbance peak1 was at $273 \mathrm{~nm}$, where $\mathrm{R}^{2}=99.99 \%$. Absorbance peak2 was at $232 \mathrm{~nm}$, where $\mathrm{R}^{2}=$ $97.80 \%$. Figure 35 shows an example of DOX concentration serial dilution absorbance vs. concentration chart from Trial \#11. The starting concentration was $50 \mu \mathrm{g} / \mathrm{ml}$, and the dilution gradient was $75 \%$. Absorbance peak1 was at $502 \mathrm{~nm}$, where $\mathrm{R}^{2}=98.37 \%$. Absorbance peak2 was at $232 \mathrm{~nm}$, where $\mathrm{R}^{2}=99.79 \%$. Figure 36 shows an example of sucrose concentration serial dilution absorbance vs. concentration chart from Trial \#9. 
The starting concentration was $50 \mu \mathrm{g} / \mathrm{ml}$, and the dilution gradient was $75 \%$. Absorbance peak1was at 292nm, and absorbance peak2 was at $240 \mathrm{~nm}$.

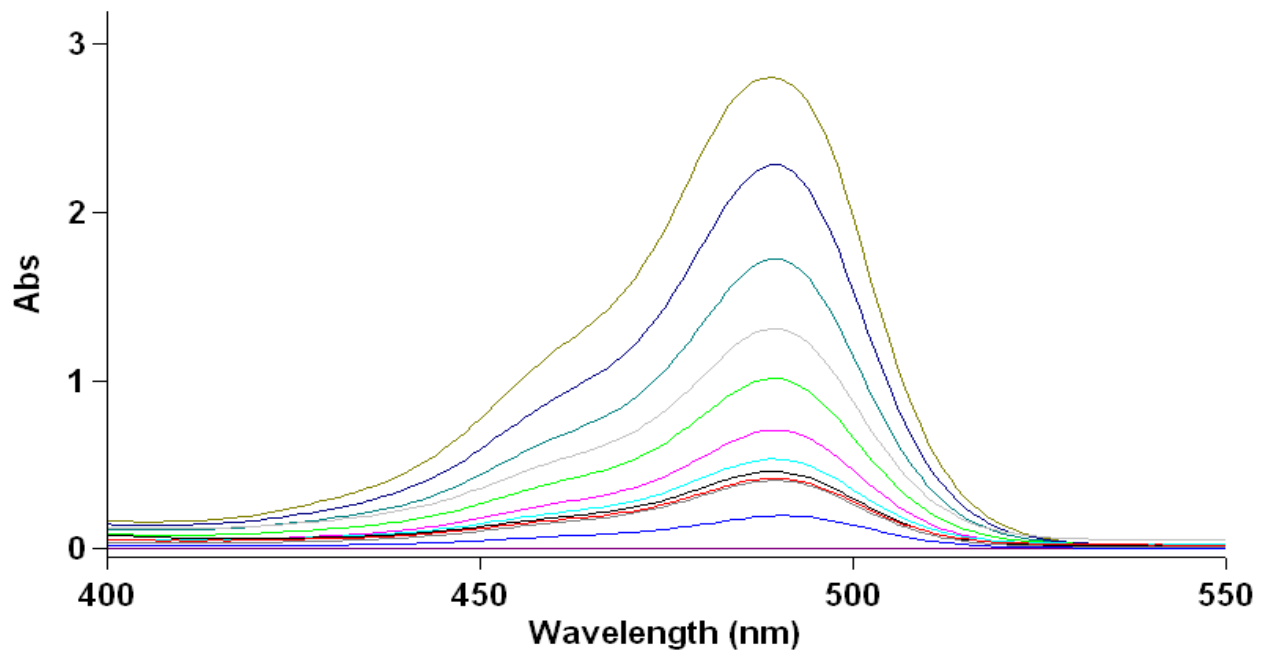

Figure 32 Example of calcein concentration serial dilution scans

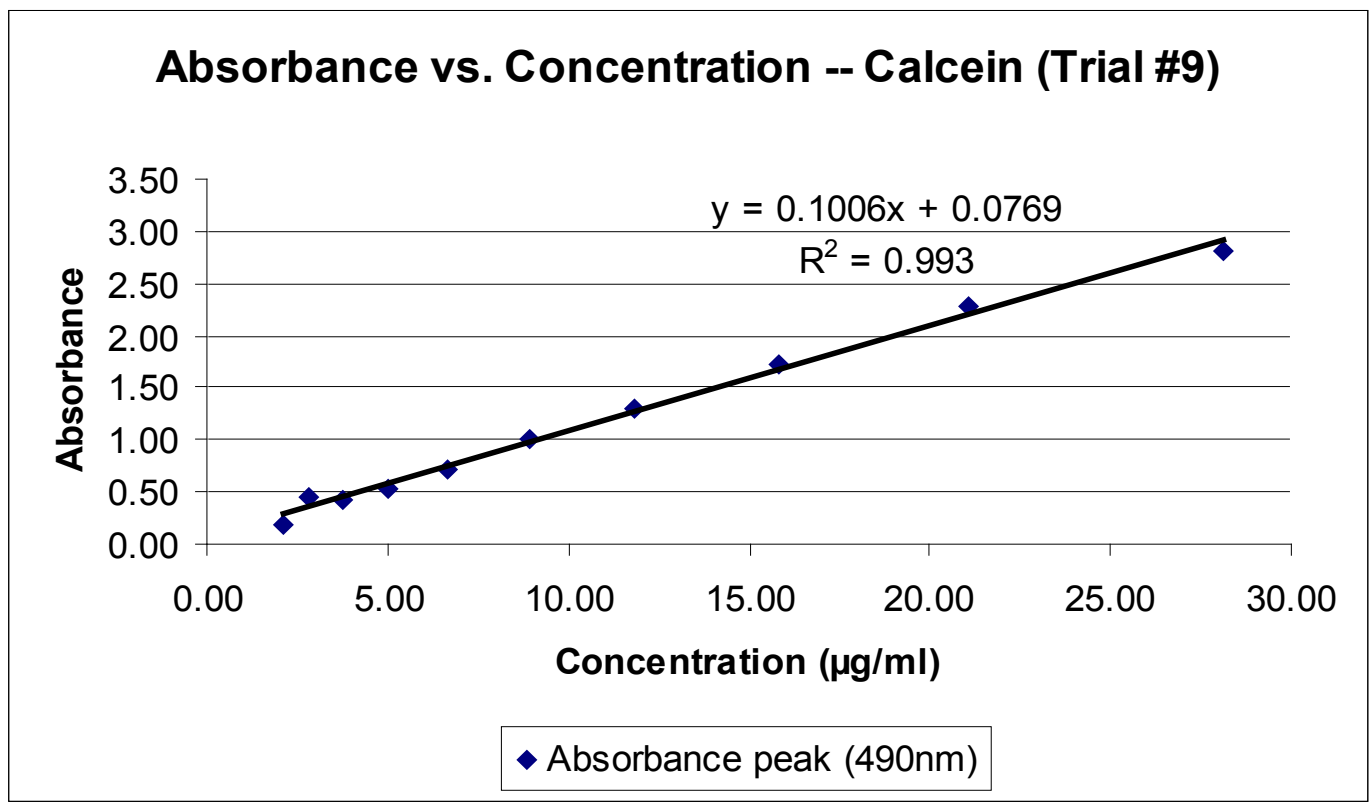

Figure 33 Example of calcein concentration serial dilution Absorbance vs.

Concentration chart 


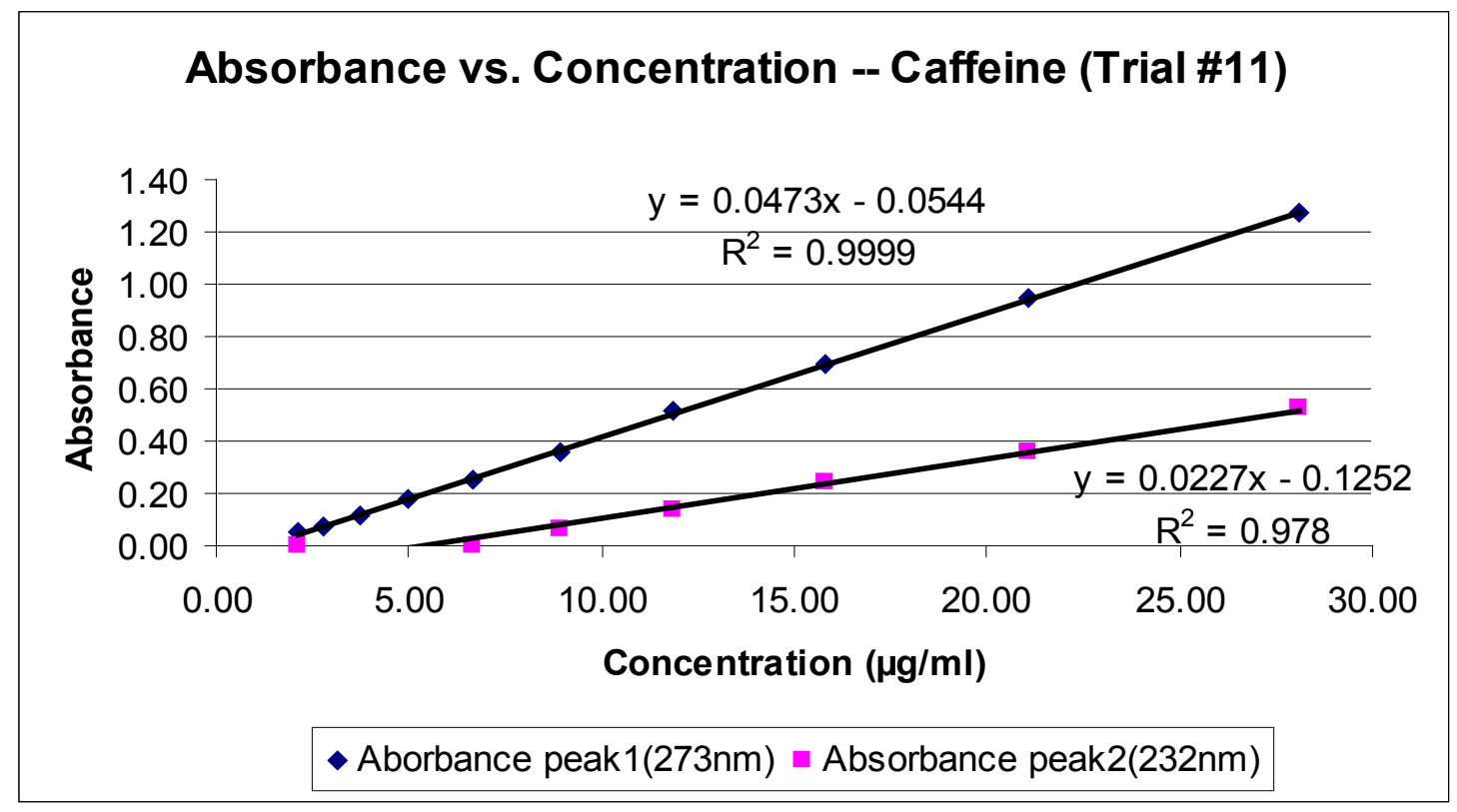

Figure 34 Example of caffeine concentration serial dilution Absorbance vs. Concentration chart

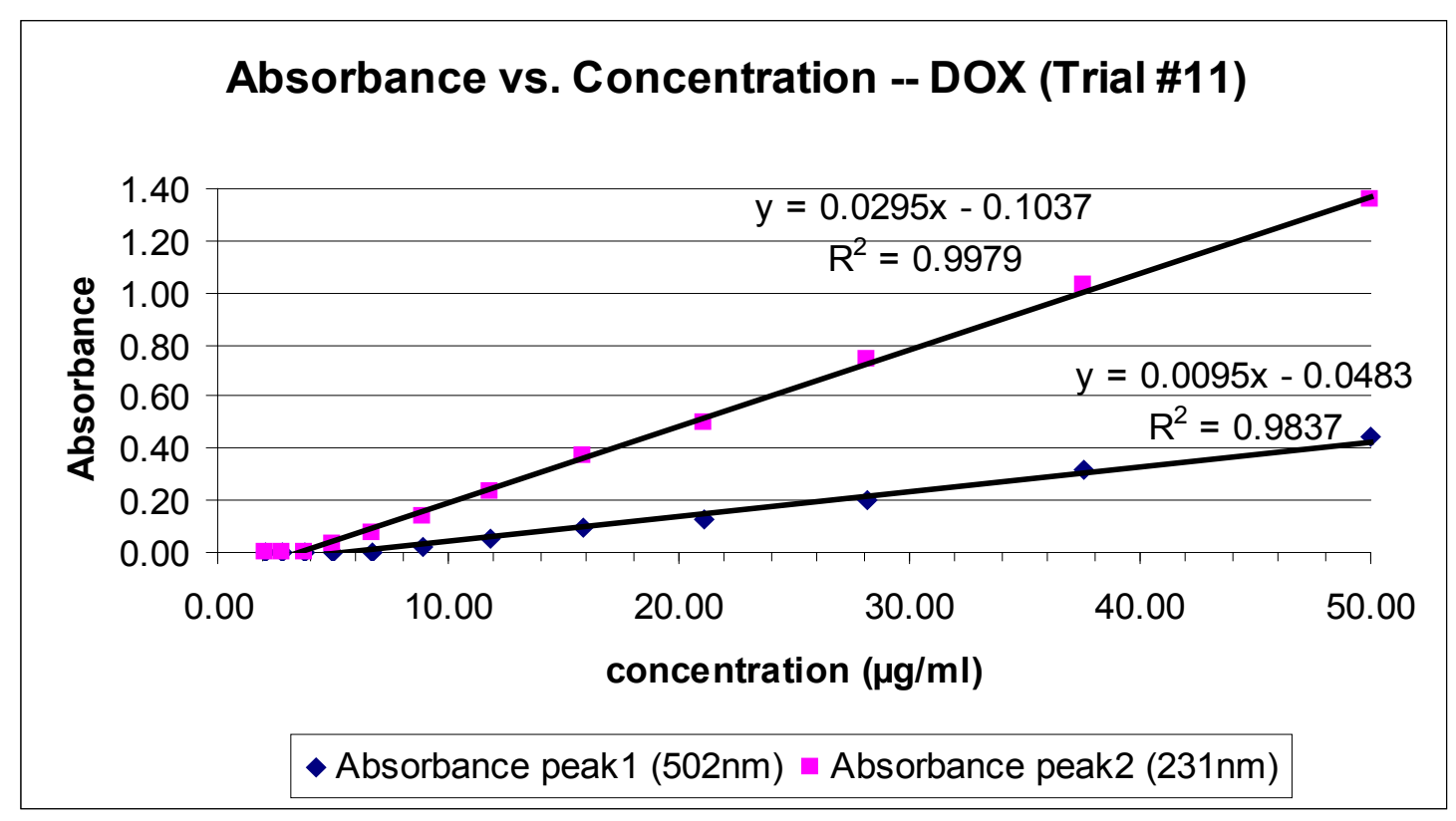

Figure 35 Example of DOX concentration serial dilution Absorbance vs.

Concentration chart 


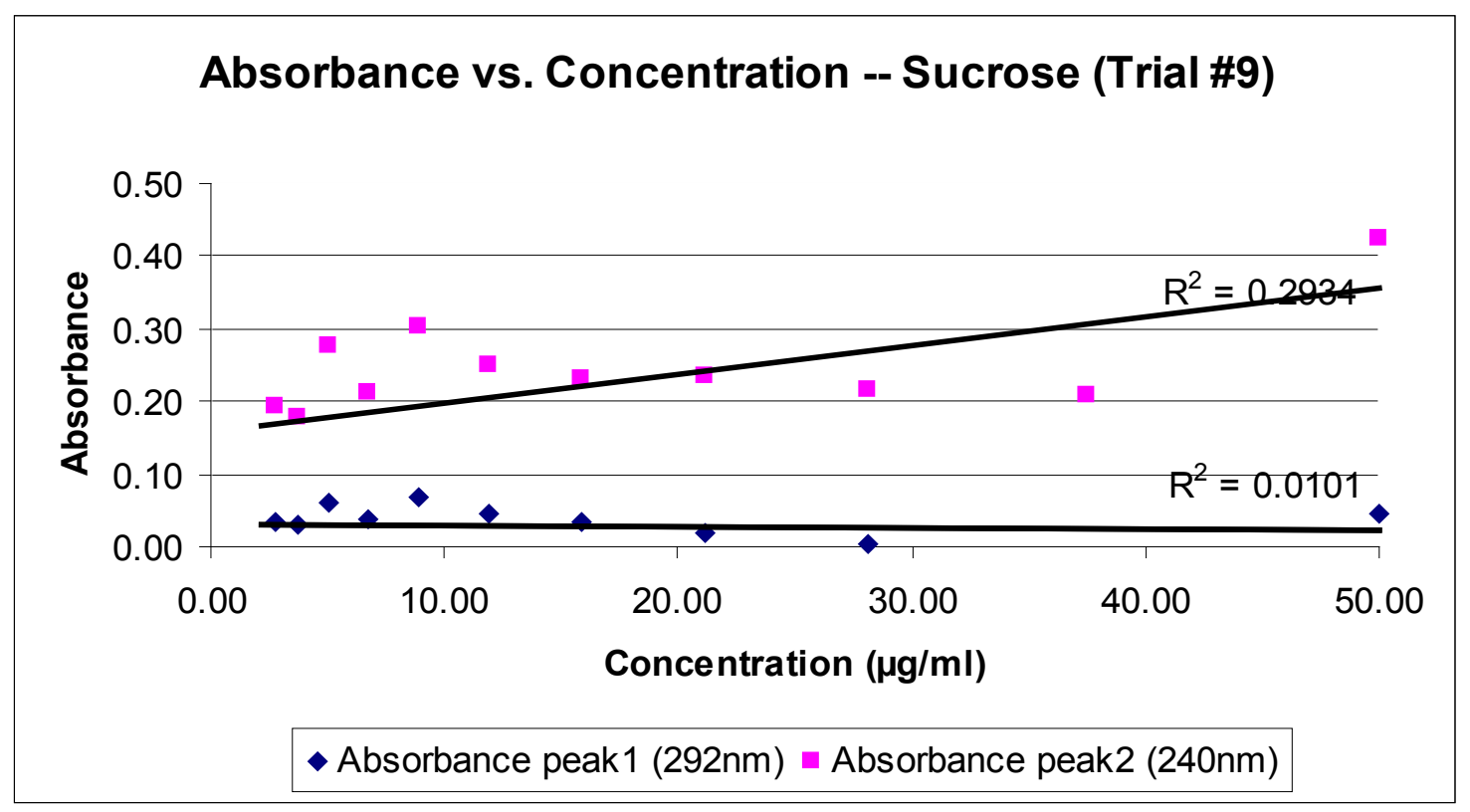

Figure 36 Example of sucrose concentration serial dilution Absorbance vs.

\section{Concentration chart}

The serial dilution curves indicated that several compounds were not suitable to be used as test candidates in the particular experiment. Sucrose and Vincristine simply did not show meaningful in optical absorbance readings between different concentrations. An example of such case is shown in Figure 36 for sucrose solution. Nicotine was an oily liquid, and was very difficult to clean from the quartz cuvette. Since the same cuvette would have to be reused throughout the experiment to eliminate the error between different cuvettes, washing became a very important procedure in the experiment. The author found once contacted nicotine, it would take more than 10 minutes to clean the cuvette with multiple rinses and solvent. Considering the large amount of samples waiting to be read and the tiny amount of the sample sizes, the samples could dry out while waiting. So nicotine was finally excluded from the candidate test compound list in order to save time and most importantly, to eliminate potential error caused by the 
changing of the sample solution volumes. Finally, calcein, caffeine and DOX were chosen to participate in the solute transport studies.

The solute concentrations of the extracted samples were calculated based on the corresponding calibration curves obtained in each trial. Mass was calculated based on the solute concentration. Effective permeability coefficients were calculated subsequently using following equation.

$$
P_{e}=\frac{V}{A}\left[\frac{M_{R} / M_{D}}{t}\right]
$$

$\mathrm{V}$ is the volume of the donor solution in the upper chamber, $\mathrm{A}$ is the surface area of the insert membrane, $M_{R}$ is the mass of the test compound in the lower chamber (mol), $M_{D}$ is the mass of the donor compound in the upper chamber (mol), and $t$ is the time (sec). Even though compensation volume of liquid was removed each time from both chambers to maintain the liquid level, the mass was calculated based on the initial volume because concentration was the value to be recorded, which would not be affected by the changing of the volume. No group has reported solute transport for the selected test compounds in similar setups, and the mass transport profiles are more of the investigation focus.

\subsection{Results and Discussions}

\subsubsection{Transport of Calcein}

Calcein $(\mathrm{MW}=622.55)$ had UV absorption peak at around 490nm and was used as a reference indicator in the experiments to evaluate the compound transport across different sample membranes. Calcein permeability profile is shown in Figure 37. All the samples with cells prevented more calcein transport over time across the membrane 
comparing to the blank insert membranes. Both of the co-cultured samples demonstrated greater ability to prevent calcein from permeating across the membranes over the time compared with ECs only sample. The "CO+aECM" samples prevented most amount of calcein from diffusing across the sample membrane. One-way ANOVA analysis showed that all the three samples setups were significantly different from each other over time (when $\alpha=0.05, \mathrm{P}_{15 \min }=1.66 \times 10^{-6}, \mathrm{P}_{30 \min }=2.42 \times 10^{-9}, \mathrm{P}_{45 \min }=1.71 \times 10^{-9}, \mathrm{P}_{60 \min }=$ $\left.3.67 \times 10^{-9}, \mathrm{P}_{90 \mathrm{~min}}=1.01 \times 10^{-8}, \mathrm{P}_{120 \mathrm{~min}}=2.25 \times 10^{-8}\right)$. All the samples were significantly different from the blank control over time (when $\alpha=0.05, \mathrm{P}_{15 \min }=4.58 \times 10^{-10}, \mathrm{P}_{30 \min }=$ $\left.2.66 \times 10^{-5}, \mathrm{P}_{45 \min }=1.65 \times 10^{-5}, \mathrm{P}_{60 \min }=1.08 \times 10^{-5}, \mathrm{P}_{90 \min }=4.78 \times 10^{-6}, \mathrm{P}_{120 \min }=3.34 \times 10^{-6}\right)$. Calcein is considered "inert" regarding to the $\mathrm{BBB}$, the transport profile demonstrated in this particular study is a proof of the passive transport of calcein across the BBB, where the solute transport is related to the impedance from the sample membranes, which were had a lot to do with the thickness of the samples.

\subsubsection{Transport of Caffeine}

Caffeine (MW=80.7, UV absorbance peak at around 275nm) is a small molecule and is known to be distributed from plasma into the brain and central nervous system(99, 100). Caffeine permeability profile from Trial \#11,12 and 13 is collectively shown in Figure 38. No significant difference between the "EC" samples and the blank controls (when $\alpha=0.05, \mathrm{P}_{15 \min }=0.0499, \mathrm{P}_{30 \min }=0.1612, \mathrm{P}_{45 \min }=0.1118, \mathrm{P}_{60 \min }=0.0649, \mathrm{P}_{90 \min }=$ $\left.0.7130, \mathrm{P}_{120 \mathrm{~min}}=0.0918\right)$. No significant difference between the "CO" \& "CO+aECM" samples (when $\alpha=0.05, \mathrm{P}_{15 \min }=0.6927, \mathrm{P}_{30 \min }=0.5030, \mathrm{P}_{45 \min }=0.3997, \mathrm{P}_{60 \min }=$ $\left.0.7548, \mathrm{P}_{90 \min }=0.6773, \mathrm{P}_{120 \min }=0.6849\right)$. Co-culture samples were significantly different from the "EC" samples (when $\alpha=0.05, \mathrm{P}_{15 \min }=0.0009, \mathrm{P}_{30 \min }=0.0001, \mathrm{P}_{45 \min }$ 
$\left.=0.0019, \mathrm{P}_{60 \min }=0.0049, \mathrm{P}_{90 \min }=0.0032, \mathrm{P}_{120 \min }=0.0254\right)$. Caffeine is reported to be actively transported across the BBB, and the data in this study demonstrated this fact, where the transport profiles between all four samples were close. Even though there were significant differences between groups, the $\mathrm{p}$ value was not as small as compare to the numbers in the calcein study. And the different between "CO" and " $\mathrm{CO}+\mathrm{aECM}$ " samples were really negligible.

\subsubsection{Transport of DOX}

Doxorubicin (DOX, MW=579.98) is a chemotherapy agent and a known substrate of p-glycoprotein on the BBB (101). In the experiment, DOX was used as one of the drug candidates to test the transport across the in vitro model sample membranes. Calcein permeability profile from Trial \#11,12 and 13 is collectively shown in Figure 39. Oneway ANOVA analysis revealed that all the three samples setups were significantly different from each other $\left(\right.$ when $\alpha=0.05, \mathrm{P}_{15 \min }=1.72 \times 10^{-6}, \mathrm{P}_{30 \min }=1.69 \times 10^{-6}, \mathrm{P}_{45 \min }=$ $\left.2.31 \times 10^{-6}, \mathrm{P}_{60 \min }=4.27 \times 10^{-6}, \mathrm{P}_{90 \min }=1.08 \times 10^{-5}, \mathrm{P}_{120 \mathrm{~min}}=0.0001\right)$. All of the samples were also significantly different from the blank control (when $\alpha=0.05, \mathrm{P}_{15 \min }=8.04 \times 10^{-}$ ${ }^{8}, \mathrm{P}_{30 \min }=6.05 \times 10^{-8}, \mathrm{P}_{45 \min }=5.16 \times 10^{-8}, \mathrm{P}_{60 \min }=5.92 \times 10^{-8}, \mathrm{P}_{90 \min }=7.48 \times 10^{-8}, \mathrm{P}_{120 \min }=$ $\left.9.25 \times 10^{-8}\right)$. Four out of the six of the "' $\mathrm{CO}+\mathrm{aECM}$ " samples prevented significantly more DOX from permeating through the membrane than the "CO" samples (when $\alpha=$ $0.05, \mathrm{P}_{15 \min }=0.0245, \mathrm{P}_{30 \min }=0.0616, \mathrm{P}_{45 \min }=0.0444, \mathrm{P}_{60 \min }=0.0422, \mathrm{P}_{90 \min }=0.0456$, $\left.\mathrm{P}_{120 \min }=0.0657\right)$. 


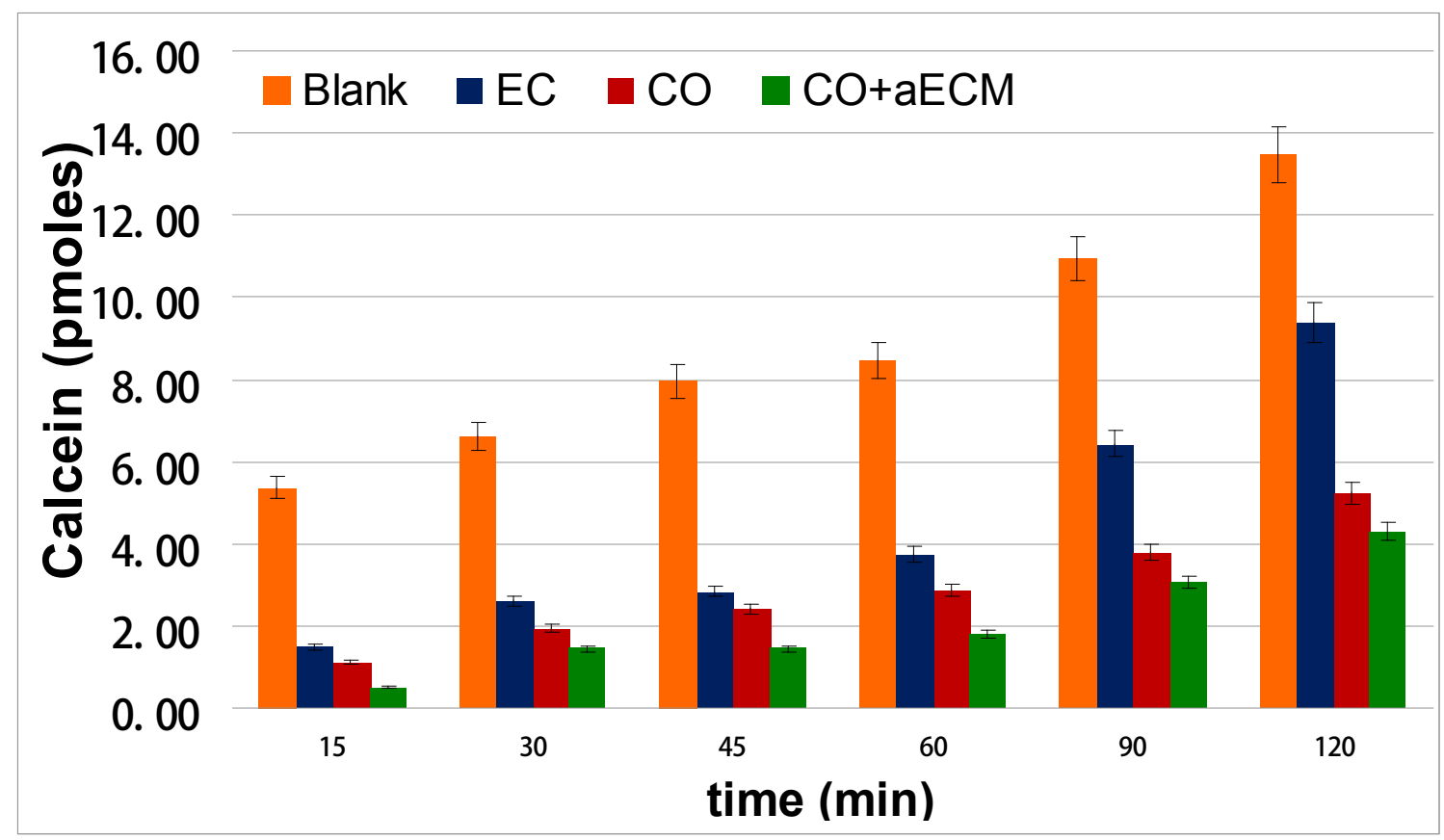

Figure 37 Calcein mass transport profiles.

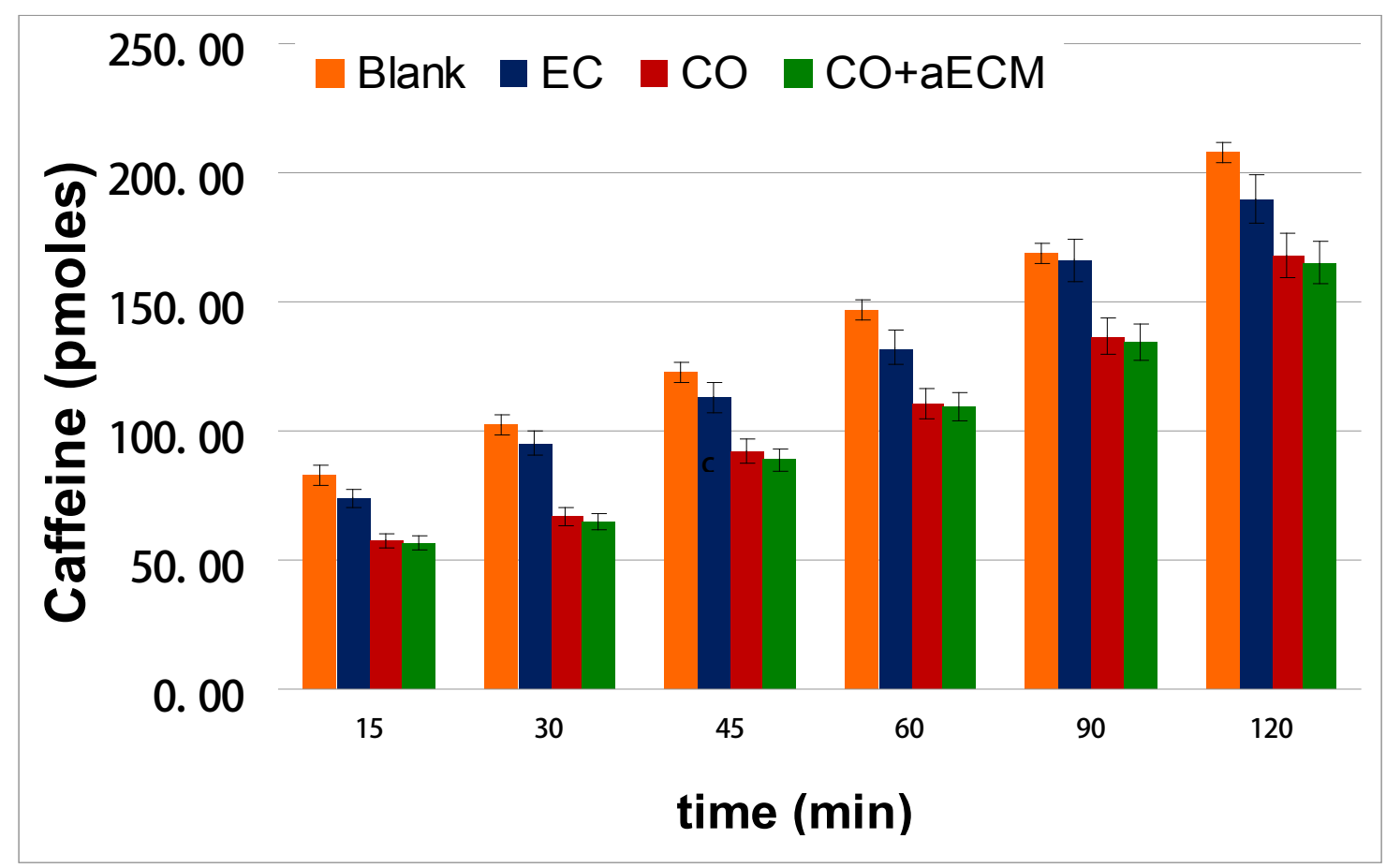

Figure 38 Caffeine mass transport profiles. 


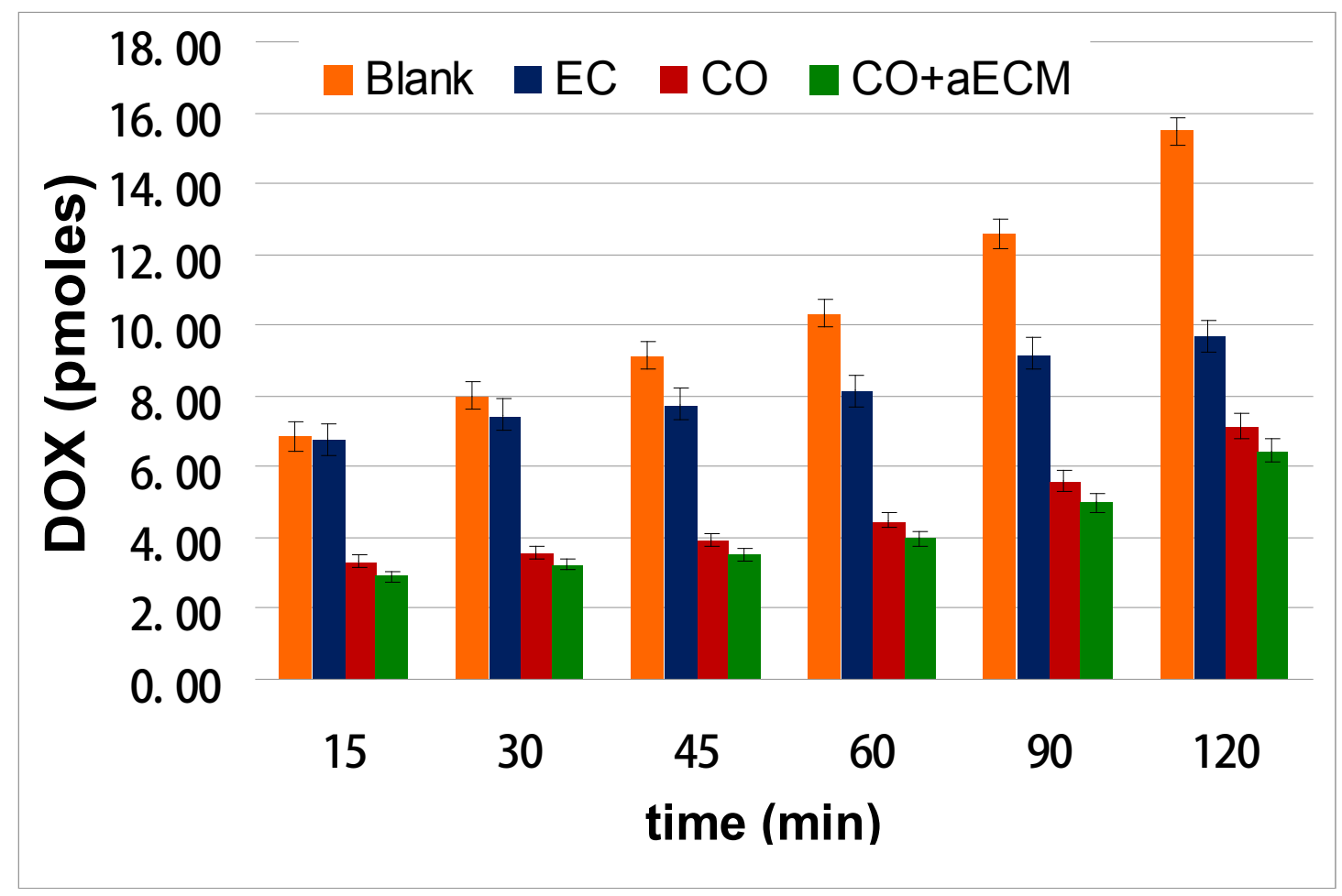

Figure 39 DOX mass transport profiles.

Since no group has reported solute transport for the selected test compounds in similar setups, the comparison of the mass transport profiles between different sample setups is more of the investigation focus. Since calcein hasn't found to be reactive with the $\mathrm{BBB}$, and its molecular weight is around the threshold, it was employed in the transport assessment because of its excellent optical absorbance calibration curves. The amounts of calcein transported across the samples membranes were used as an indicator for molecules of its size. Statistical analysis showed that all the sample setup was different between each other, and the "CO+aECM" samples prevented the most amount of calcein transport with the effective permeability coefficient equaled to $(1.33 \pm 0.25)$ $\times 10^{-6} \mathrm{~cm} \mathrm{sec}^{-1}$. As a small molecule with its molecular weight well below the threshold, the amounts of caffeine transported across the membranes were much more than those of 
calcein. However, there was no significant difference found between the "EC" samples and the "Blank" samples. or between the "CO" samples and the "CO+aECM" samples. Even though caffeine solute transport through the "CO" or "CO+aECM" samples was statistically significantly lower than that of the "EC" or "Blank" samples, the p-values were not as low as those in comparison in the calcein transport profiles. The effective permeability coefficients for caffeine in the different setups were almost four times of those of calcein's. These results were not surprising because caffeine was reported to be actively transported across BBB by saturable mediated processes and the results verified the theory by the high efficient permeability coefficient $[30,31]$. The assessment here served as an example of the caffeine solute transport across the BBB. For the DOX transport profiles, the transport profiles of both the "CO" and the "CO+aECM" samples were statistically significantly lower than that of the "EC" samples. And the majority of the "CO+aECM" samples prevented significantly more DOX from being transported across the sample membranes than those of the "CO" samples. The next step for the authors would be to evaluate the existence of p-glycoproteins on the sample membranes, but for now the available results could be concluded as a phenomenal proof of the existence of some DOX-resistant component on the co-culture sample membranes, which were found to be more on the "CO+aECM" samples than those on the "CO" samples.

\subsubsection{Permeability}

The effective permeability coefficients of different test compounds across different sample setups are summarized in Table 6 . The values are expressed in average \pm standard deviation for trails \# 11, 12, and 13. The permeability coefficients were 
comparable to those found in Garbergs et al.'s various BBB in vitro model attempts, thus proving that the samples had the potential to serve as relevant BBB in vitro model. The only comparable value from literature is the caffeine in vivo "mouse brain uptake assay" where the apparent permeability coefficient is $(8.1 \pm 1.2) \times 10^{-6} \mathrm{~cm} / \mathrm{s}$, which is close to the values from the "CO" samples.

Table 6 Permeability of different test compounds across different sample setups

\begin{tabular}{|c|c|c|c|}
\hline $\mathrm{P}_{\mathrm{e}}\left(\times 10^{-6} \mathrm{~cm} \mathrm{sec}^{-1}\right)$ & Calcein & Caffeine & DOX \\
\hline "EC" samples & $2.90 \pm 0.55$ & $11.07 \pm 3.02$ & $4.87 \pm 1.18$ \\
\hline "CO" samples & $2.05 \pm 0.48$ & $8.33 \pm 1.45$ & $2.66 \pm 0.80$ \\
\hline "CO+aECM" samples & $1.33 \pm 0.25$ & $8.14 \pm 1.61$ & $2.38 \pm 0.73$ \\
\hline Blank controls & $7.10 \pm 0.35$ & $14.44 \pm 0.68$ & $8.61 \pm 0.46$ \\
\hline
\end{tabular}

The transport profiles and permeability coefficient values look better than the results from the TEER measurement in terms of closeness to the in vivo values. However, this does not mean there is inconsistency between these two sets of measurements. In fact, after the transport sampling procedures for calcein, a noticeable amount of calcein could be found to be retained by the sample membranes by showing of the fluorescent yellow color. This could result in lower reading of actual amount of transported solute, and thus the less detectable solute in the transport studies.

\subsection{Summary}

This chapter looked at solute mass transport through diffusion for calcein, caffeine and DOX. The membranes allow the small molecule caffeine, which was also actively transported by the BBB to pass through, while prevented in proportion of calcein 
and DOX. However, the membranes reacted differently between calcein and DOX. The mass transport for calcein solutions followed more of a time and concentration dependent route. While the co-cultures were able to prevent significant amount of DOX from passing through the membrane while "EC" samples permitted much more DOX to go through. The comparable effective permeability coefficients of the co-culture samples demonstrated they may serve as potential relevant $\mathrm{BBB}$ in vitro models. 


\section{CHAPTER VIII TIGHT JUNCTION PROTEIN EXPRESSION}

\subsection{Western Blot Analysis}

As introduced in previous chapters, there are several specific proteins exist on the $\mathrm{BBB}$ endothelial tight junctions. Occludin is one of the earliest identified tight junction protein. The author employed western blot analysis to examine the expression of occludin on different samples in the experiments. The selected samples included EC, CO and $\mathrm{CO}+\mathrm{aECM}$ samples. Detailed procedures and results are described in the following sections.

\subsubsection{Introduction to Western Blot Techniques}

Western blot is a technique to detect a specific protein from a given mixture of proteins from tissue extract or lysate. It is a technique composed of gel electrophoresis and immunochemical detection. This technique is very useful to identify a protein from a complex mixture of proteins that are not radio labeled.

Sodium dodecyl sulfate polyacrylamide (SDS-PAGE) gel electrophoresis is the most popular technique used by the scientists to electrophoretically separate denatured proteins according to their molecular weight. The sieving property of the SDS-PAGE gel electrophoresis is based on the absolute concentrations of acrylamide and bisacrylamide, which determine the size of the pores on the gel. SDS is a highly negatively charged detergent employed to proportionally bind to the protein according to their molecular weight. Thus during the gel electrophoresis, the SDS-bound protein complexes migrate toward the anode side, during which the larger the protein size, the slower it moves. So at the end of the gel electrophoresis, proteins are separated and distributed on different locations of the gel. 
After the proteins are electrophoretically separated, they are transferred onto a piece of nitrocellulose membrane in the next step. Primary antibody will be applied to the membrane to detect the target protein. The primary antibody is usually raised in a different host species against the target protein. It can also be obtained from immune cell culture which is exposed to the target protein.

In order to prevent interactions between the membrane and the primary antibody for the detection of the target protein, $5 \%$ bovine serum albumen (BSA) or non-fat dry milk are employed to soak the membrane before the application of the primary antibody. The blocking procedure makes sure the areas on the membrane which are not attached by the target protein are attached by the inert BSA or the milk proteins. Thus when the primary antibody is applied, it will only bind to the target protein and the background noise is significantly reduced.

In the next step, secondary antibody is employed to detect the primary antibody for a species-specific portion on it, e.g. anti-mouse secondary antibody will detect mousesourced primary antibody. The secondary antibodies are usually associated with a reporter enzyme such as, most commonly, horseradish peroxidase (HRP) for further detection. The HRP on the secondary antibody can cleave a chemiluminescent agent such as lunimol, which is able to exhibit chemiluminescence in proportion to the amount of the protein. Thus in a dark room, the generated chemiluminescence from the nitrocellulose membrane can expose a photographic film to reveal the expression level of the target protein. 


\subsubsection{Methods}

In the end of the culture period, cell layers from trial \#7 were scraped from the upper sides of the inserts from $\mathrm{EC}$ only, $\mathrm{CO}$ and $\mathrm{CO}+\mathrm{aECM}$ samples, respectively. 4 replications from each sample were dedicated to contribute cells for this study. The cell mixtures were solubilized with lysis buffer containing 5mM EDTA, 10mM Tris buffer, $125 \mathrm{mM} \mathrm{NaCl}, 1 \%$ Triton-X 100, and protease inhibitor cocktail (Thermo Scientific). The cell lysates were subjected to $10 \%$ SDS-PAGE resolving gel electrophoresis. The $10 \%$ resolving gel was capable of separating proteins in the molecular range of 19-90 $\mathrm{kDa}$, casted by mixing $4 \mathrm{ml} \mathrm{H}_{2} \mathrm{O}, 3.3 \mathrm{ml} 30 \%$ acrylamide, $2.5 \mathrm{ml} 1.5 \mathrm{M}$ Tris $(\mathrm{pH}=8.8)$, $100 \mu \mathrm{l}$ SDS, $100 \mu 1 \quad 10 \%$ APS (ammonium persulfate solution) and $4 \mu 1$ TEMED ( $N, N, N^{\prime}, N^{\prime}$-tetramethylenediamine) (Sigma-Aldrich). The recipe for the stacking gel was: $3.4 \mathrm{ml} \mathrm{H} \mathrm{H}_{2} \mathrm{O}, 830 \mu \mathrm{l} 30 \%$ acrylamide, $630 \mu \mathrm{l} 1.0 \mathrm{M}$ Tris $(\mathrm{pH}=6.8), 50 \mu 110 \%$ SDS, $50 \mu 1$ $10 \%$ APS and $5 \mu 1$ TEMED.

$2 \mu 1$ of the protein lysates, $18 \mu 1 \mathrm{H}_{2} \mathrm{O}$ and $20 \mu 12 \times$ SDS gel loading buffer (Fisher Scientific) were mixed and heated at $95^{\circ} \mathrm{C}$ for 5 minutes in a dry bath. The heating procedure caused the proteins to unfold and bound to the negatively charged SDS molecules. The heated protein mixtures were placed on ice to cool down for 3-5 minutes and then, $15 \mu 1$ of each protein mixture was loaded into designated lanes on the gel, respectively. $10 \mu 1$ of EZ-Run ${ }^{\mathrm{TM}}$ pre-stained protein ladder (Fisher BioReagents) was loaded into a lane in the same gel to provide molecular weight indications. Alongside the protein lysates, a $15 \mu \mathrm{l}$ of the occludin recombinant protein (molecular weigh $\approx 60 \mathrm{kDa}$ ) purchased from Novus Biologicals (Littleton, CO) was also added into a lane on the gel serving as positive control. The electrophoresis was run in the electrophoresis chamber 
(BioRad, Hercules, CA) at 100V for approximately 90 minutes through the end of the gel. The electrophoresis contained $0.25 \mathrm{M}$ Tris base, $1.92 \mathrm{M}$ Glycine and 20\% w/v SDS.

At the end of the electrophoresis, the gel was carefully separated from the assembly and put into the container with transfer buffer (BupH Tris-glycine buffer: when dissolved in $500 \mathrm{~mL}$ of $20 \%$ methanol, each pack yields $25 \mathrm{mM}$ Tris, $192 \mathrm{mM}$ glycine, $\mathrm{pH}$ 8. Fisher Scientific). The nitrocellulose membrane, filters and sponges were presoaked in the container for 20 minutes. A sandwich was assembled in the following order: the negative electrode, a piece of sponge, a piece of filter paper, the SDS-PAGE gel, the nitrocellulose membrane, another piece of filter paper, another piece of sponge, and finally the positive electrode. Figure 40 shows a picture of how the gel transfer sandwich was assembled during the experiment. The assembly was placed in the transfer tank (BioRad) full of transfer buffer and transferred for 1 hour at $50 \mathrm{~V}, 164 \mathrm{~mA}$ at room temperature.

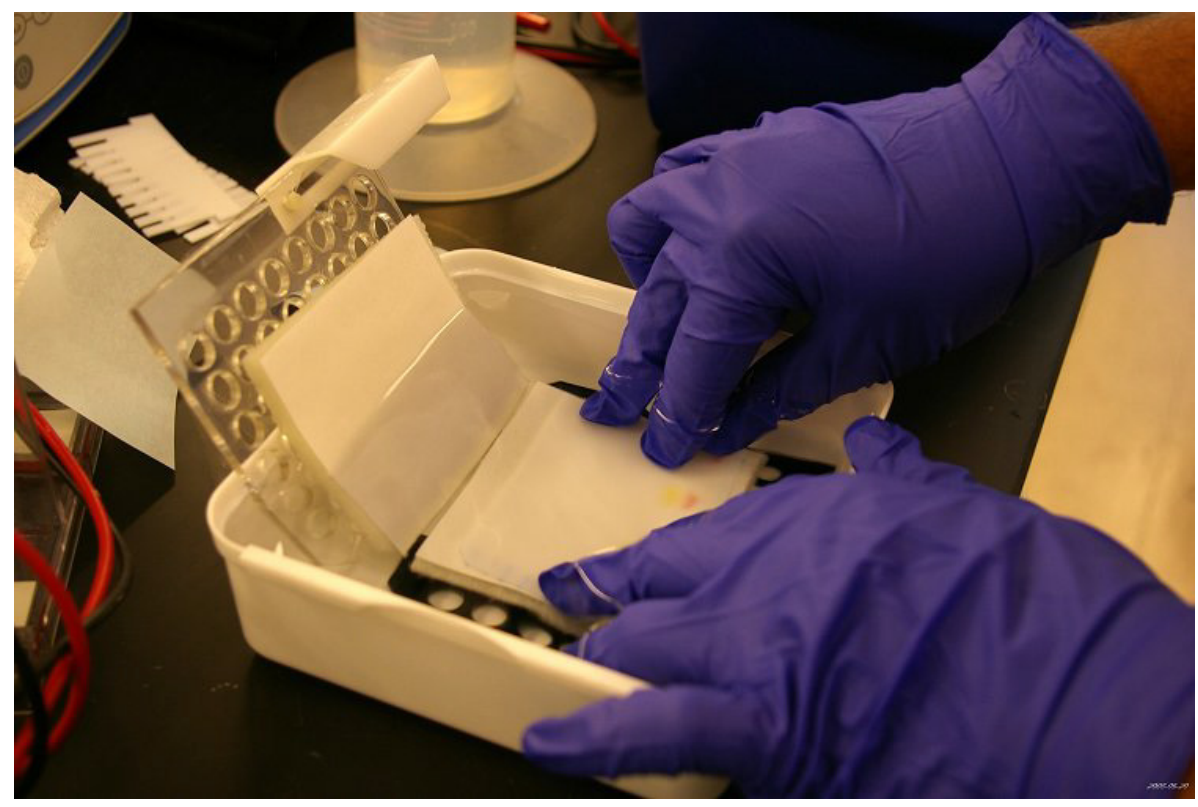

Figure 40 Sandwich assembly for Western Blot gel transfer 
In the end of the transfer procedure, proteins were transferred onto the nitrocellulose membrane. The nitrocellulose membrane was blocked with 5\% BSA (Fisher Scientific) for 1 hour with agitation. The membrane was then washed 3 times for 5 minute with TBST (Tris Buffered Saline, with Tween $^{\circledR}$ 20, Sigma-Aldrich with agitation. Then the membrane was incubated with primary antibody for 16 hours at $4{ }^{\circ} \mathrm{C}$ with agitation. The primary antibody was 1:20 $(5 \mu \mathrm{g} / \mathrm{ml})$ rabbit anti-occludin (US Biological) diluted in TBST containing 5\% BSA. The incubated membrane was washed 3 times for 5 minutes each with TBST with agitation. Then the membrane was further incubated with secondary antibody (US Biological) for another hour at room temperature with agitation. The secondary antibody was 1:10000 HRP conjugated anti-rabbit IgG diluted in TBST with 5\% BSA. Then the membrane was washed another 3 times for 5 minutes each with TBST with agitation.

The final membrane was soaked in $10 \mathrm{ml}$ of LumiGLO Reagent $(0.5 \mathrm{ml}$ 20XLumiGLO, 0.5ml 20X Peroxide, 9ml water) (Cell Signaling Technology, Inc. Danvers, MA)with gentle agitation for 1 minute. The immunoreactive bands were exposed onto Pierce CL-Xposure films (Fisher Scientific) in the dark room.

\subsubsection{Results and Discussion}

The scan of the exposed film from the experiment is demonstration as in Figure 41. Clearly the detected protein bands all showed up at around $60 \mathrm{kDa}$ of molecular weight, which was the predicted molecular weight of occludin. In the picture, lane 1 represented the protein band from the occludin recombinant protein positive control. Lane 2 represented the protein band from the protein mixture from astrocytes alone samples. Lane 3 represented the protein band from the protein mixture from EC alone 
samples. Lane 4 shows the protein band from the protein mixture from CO samples. Lane 5 shows the protein band from the protein mixture from $\mathrm{CO}+\mathrm{aECM}$ samples.

It was clear from the picture that no occludin protein band was detected from the EC alone or astrocytes alone samples. Both $\mathrm{CO}$ and $\mathrm{CO}+\mathrm{aECM}$ samples displayed detected occludin protein band on the film, while the chemiluminescent intensity of the $\mathrm{CO}+\mathrm{aECM}$ samples was apparently stronger than that of the $\mathrm{CO}$ samples. This could be interpreted as there were more occludin expressed on the $\mathrm{CO}+\mathrm{aECM}$ samples than the CO samples.

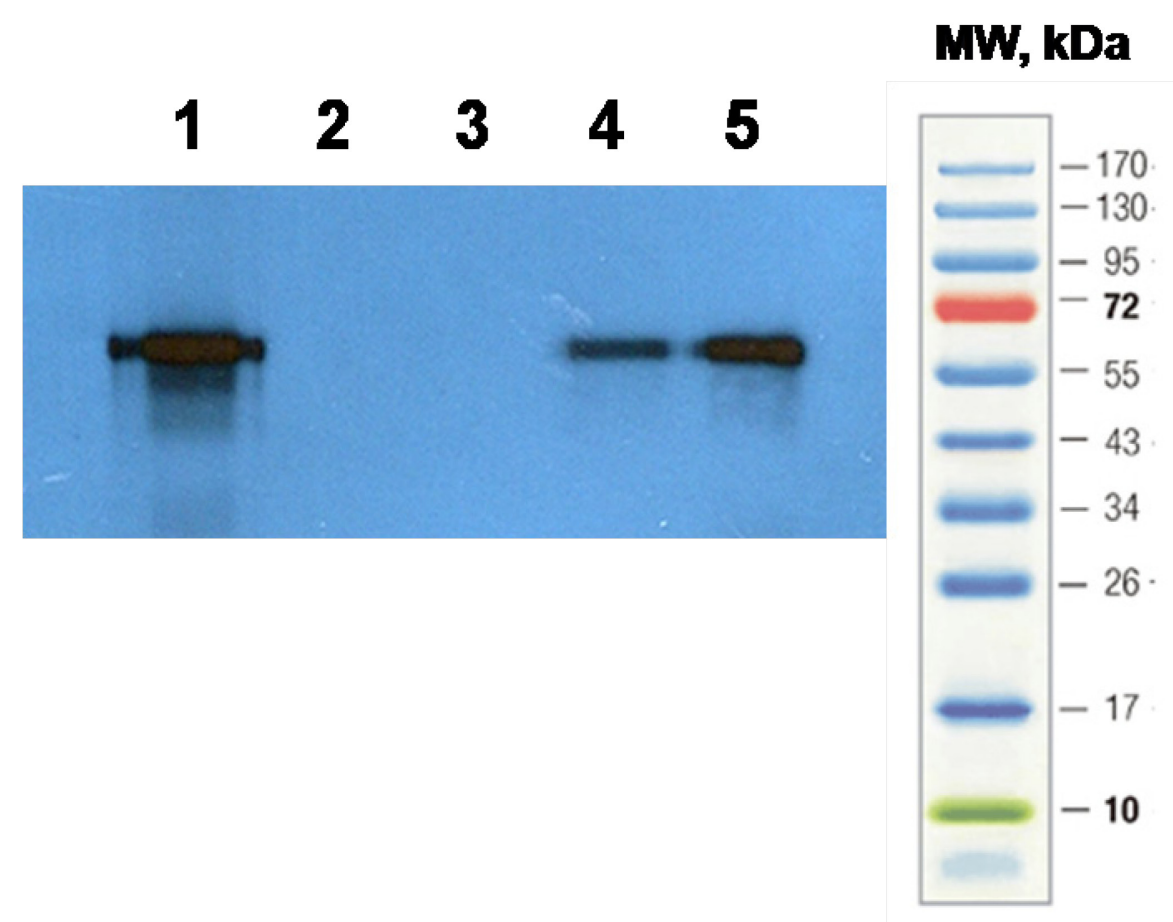

Figure 41 Scan of exposed film from the Western Blot analysis of occludin

Because of the very limited funding availability during the time of the experiment, the Western Blot analysis was only carried out once for one of the tight junction proteins, occludin itself for one trial. However, the result was obvious enough to show the different occludin express levels on different sample setups, which agreed with 
the initial assumption that the endothelial cell layers from the CO+aECM samples expressed the highest level of occludin, while endothelial cells cultured alone or astrocytes cultured alone were not able to express enough level of occludin if there was any, to be detected by this effective protein detection method. The endothelial cell layers from the $\mathrm{CO}$ samples were able to express a level of occludin in this analysis, but the amount was smaller than that from the CO+aECM samples.

\subsection{Immuno-Fluorescence Microscopy}

The expression of occludin was further examined by immuno-fluorescence microscopy. Since there are many similarities between immuno-fluorescence microscopy and Western Blot analysis, much briefer language will be used to describe this method in the following sections.

\subsubsection{Introduction to Immuno-Fluorescence Microscopy}

Immuno-fluorescence microscopy method is usually used to visualize the subcellular allocation of biomolecules of interest. Similar to the Western Blot analysis, two types of antibodies are included in immuno-fluorescence microscopy studies: the primary antibody is usually raised in another species against the target protein to detect the protein; the primary protein will be recognized by the secondary protein which is conjugated with a fluorescent dye. The examples of fluorescent dyes are fluorescein and rhodamine. At last the immuno-fluorescent labeled culture samples can be studies using a fluorescence microscope or a confocal microscope.

\subsubsection{Methods}

Select samples from Trial \#8 were chosen for the immuno-fluorescence microscopy study. The samples included EC, CO and CO+aECM. At the end of the 
culturing period, cell layers were treated immediately with ice cold $0.2 \%$ Triton X-100 for 2 minutes after removing the culture media. After the immediate treatment, the cell layers were fixed directly on the culture inserts by gently adding 3\% paraformaldehyde (Sigma-Aldrich) directly into the inserts. The cell layers were being fixed for 30 minutes at room temperature. After fixation, the samples were treated with ice cold $0.05 \%$ Triton X-100 in PBS for 5 minutes. Then, the samples were blocked by 5\% BSA solution in room temperature for 1 hour. After blocking, the samples were incubated with 1:20 rabbit anti-occludin primary antibody (US Biological) in room temperature for 1 hour. The samples were further incubated with 1:5000 FITC-conjugated goat anti-rabbit IgG (US Biological) in room temperature for 30 minutes. FITC is Fluorescein isothiocyanate, a derivative of fluorescein and is widely used in immuno-fluorescence microscopy. The samples were examined by IX81 Motorized Inverted Microscope (Olympus) at the excitation/emission wavelength at $495 \mathrm{~nm} / 521 \mathrm{~nm}$.

\subsubsection{Results and Discussion}

The immuno-staining results for $\mathrm{CO}$ and $\mathrm{CO}+\mathrm{aECM}$ samples are shown in Figure 42. The fluorescence image of EC sample was completely dark so it was not shown here. Tight junction protein occludin's contours could be observed from both co-cultured sample setups. However, the cells contours marked by the stained occludin were more obvious on the $\mathrm{CO}+\mathrm{aECM}$ samples than those of the $\mathrm{CO}$ samples. There was a blurred pattern of green color on Figure 42(A) which could be the noise coming from the PTFE fibers from below the cell layer but not the stained protein itself. The cell silhouettes on Figure 42(B) were much easier to distinguish than those on Figure 42(A) because of the higher density of the stained occludin on the CO+aECM samples. The results indicated 
more occludin was expressed on the $\mathrm{CO}+\mathrm{aECM}$ sample than on the $\mathrm{CO}$ sample, while there was no detectable occludin on the ECM sample. Astrocytes in both co-culture samples helped induce the endothelial cells to express the tight junction protein occludin, while the aECM was important for the endothelial cells to form better tight junctions as shown in Figure 42.
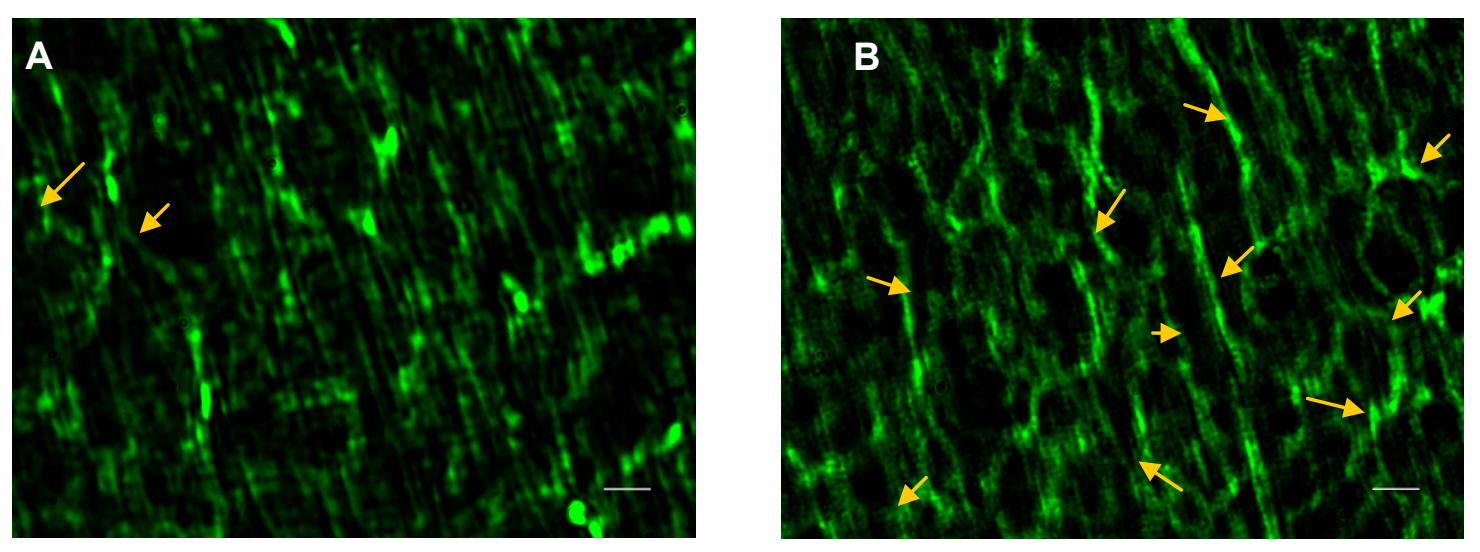

Figure 42 Immuno-fluorescence microscopy image of endothelial layers from coculture samples: (A): CO sample; (B): $\mathrm{CO}+\mathrm{aECM}$ sample

\subsection{Summary}

This chapter focuses on the expression of occludin, one of the earliest identified proteins on $\mathrm{BBB}$ endothelial tight junctions. Even though due to the shortage of research funding, only limited experiments were carried out for only one type of tight junction protein, the positive results from both experiments from two sets of trials are persuasive. Endothelial layers from both of the co-culture samples were capable of expressing occludin while the endothelial cells cultured alone were not able to express if any, detectable amount of occludin by this particular analysis. The $\mathrm{CO}+\mathrm{aECM}$ samples were superior to the $\mathrm{CO}$ samples in terms of occludin expression level. Because occludin does 
not exists on astrocytes, the higher occludin expression level could be accredited to the contribution of the aECM, which was assumed to help the endothelial cells exhibit more BBB capillary like characteristics including tight junction proteins expression. 


\section{CHAPTER IX ASTROCYTE LOSS ANALYSIS}

\subsection{Introduction}

In the co-culture samples, astrocytes growing on the basal sides of the inserts had to fight the gravity pull and a portion would inevitably fell onto the well bottoms where the inserts sat. The amount of astrocytes that fell from the basal sides of the insert membranes from selected co-culture samples were evaluated by in vitro colorimetric Sulforhodamine B (SRB) assay. SRB is a fluorescent dye widely used in the quantification of cell toxicity assay. The dye binds to cellular protein and when solubilized in base, the optical absorbance can be measured at the wavelength of $520 \mathrm{~nm}$ by a micro plate optical reader. By obtaining optical density readings from the micro plate reader machine, cell density can be determined based on the optical absorbance vs. cell density calibration curve carried out experimentally for each specific type of cell(102).

\subsection{Methods}

At the end of culturing period, the well bottoms of the $\mathrm{CO}$ and $\mathrm{CO}+\mathrm{aECM}$ samples from Trial \#7, \#8, \#9 and \#11 on the 24-well plates were fixed for 30 min by 50\% trichloroacetic acid (TCA) purchased from Sigma-Aldrich (St. Louis, MO). According to the protocol, the fixed wells were rinsed 4 times with tap water and air dried before SRB staining. It took $30 \mathrm{~min}$ for $0.1 \%$ SRB (Sigma-Aldrich, St. Louis, MO) diluted in $1 \%$ acetic acid (Sigma-Aldrich, St. Louis, MO) to stain the dried well bottoms where the astrocytes fell onto. The stained samples were rinsed with $1 \%$ acetic acid to remove unbound dye and dried over night until no standing moisture was visible. Finally, the stained wells were solubilized by $10 \mathrm{mM}$ Trizma base (Sigma-Aldrich, St. Louis, 
MO) solution and optical absorbance was measured at 520nm by a GNEios TECAN micro plate reader (Phoenix Research, Candler, NC). The 24-well plates were shaken for 5 min before measurement to facilitate the extraction, solubilization and mixing of SRB dye molecules. The final experimental results were expressed as the number of cells calculated based on the linear relationship between the optical absorbance and cell numbers.

\subsection{Astrocytes Cell Number SRB Calibration Curve}

Cell number calibration curve for astrocytes was generated to facilitate the conversion between optical densities to cell numbers. Briefly, 6 different concentrations of astrocytes cell mixtures were seeded onto the 6 column of 24 -well plate, each well with 4 replications. The 6 different cell numbers were: $0.25 \times 10^{4}$ cells $/$ well, $0.5 \times 10^{4} \mathrm{cells} /$ well, $1 \times 10^{4}$ cells/well, $2 \times 10^{4}$ cells/well, $4 \times 10^{4}$ cells $/$ well, and $8 \times 10^{4}$ cells $/$ well . After the cells are seeded onto the well bottoms, they were allowed 24 hours in order to attach to the bottoms before fixation. Following the same procedure as aforementioned, the well bottoms were fixed, rinsed and stained by SRB. The picture shown below is a demonstration of how the SRB stained and dried well bottoms looked like for the calibration plate.

The obtained calibration curve is as following graph shows. This graph shows a linear relationship $\left(\mathrm{R}^{2}=99.86 \%\right)$ between the well cell density and the optical absorbance so it would be pertinent to use the curve fitting linear equation $\left(y=4 \times 10^{-6}\right.$ 0.001) to predict the cell numbers using optical absorbance obtained from the experiments. 


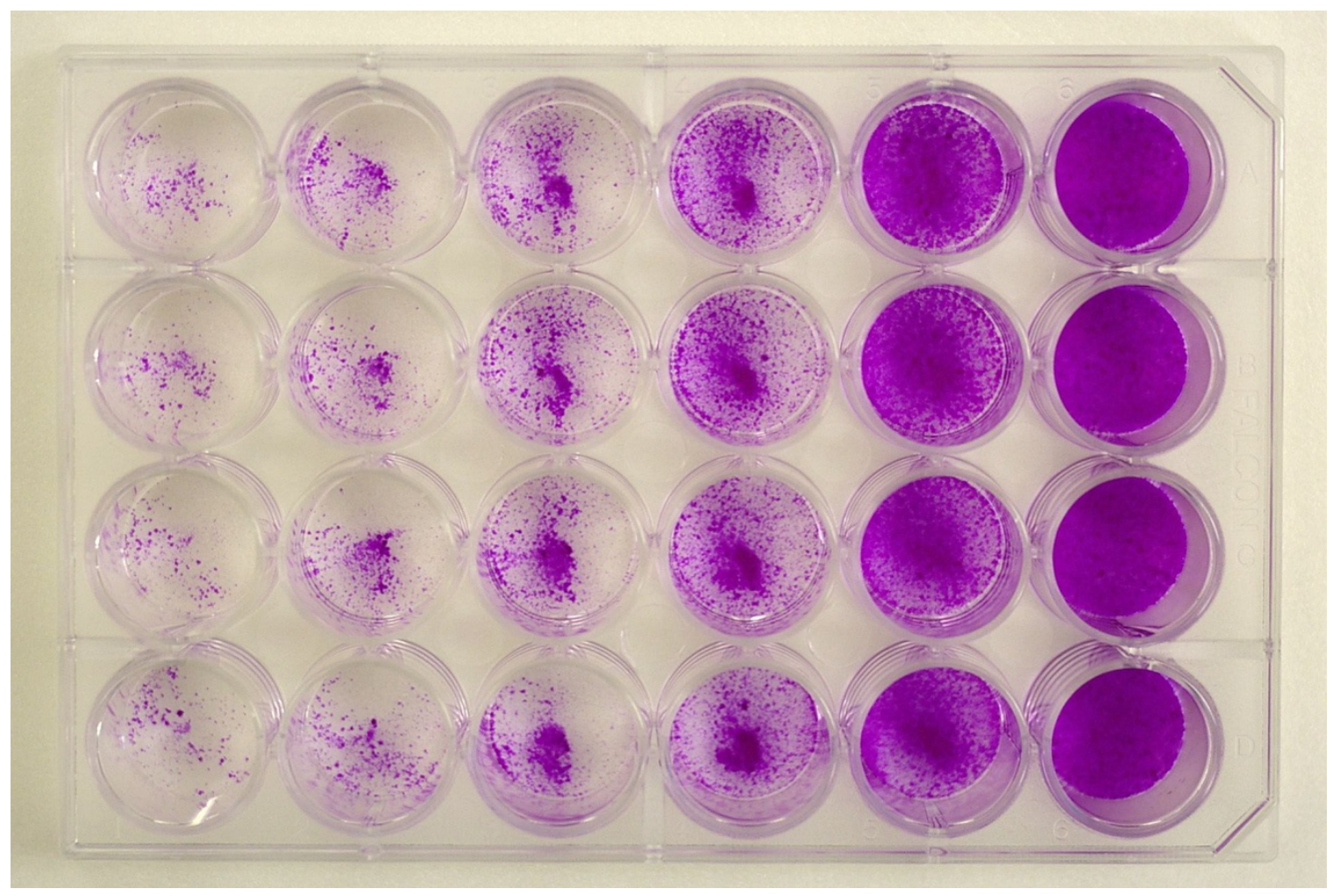

Figure 43 Dried SRB stained 24-well plate for the calibration curve

\subsection{Results}

SRB optical absorbencies from the well bottoms of 4 trials were obtained, with 4 replications of each trial, respectively. Furthermore, in order to reduce possible background noise generated from the plastic wells, the optical absorbencies were taken each time for the blank wells and the values were subtracted from the readings to get the normalized final values. The normalized optical absorbance values were then calculated using the equation obtained from the calibration curve, and the results are displayed as following table in cell numbers. 


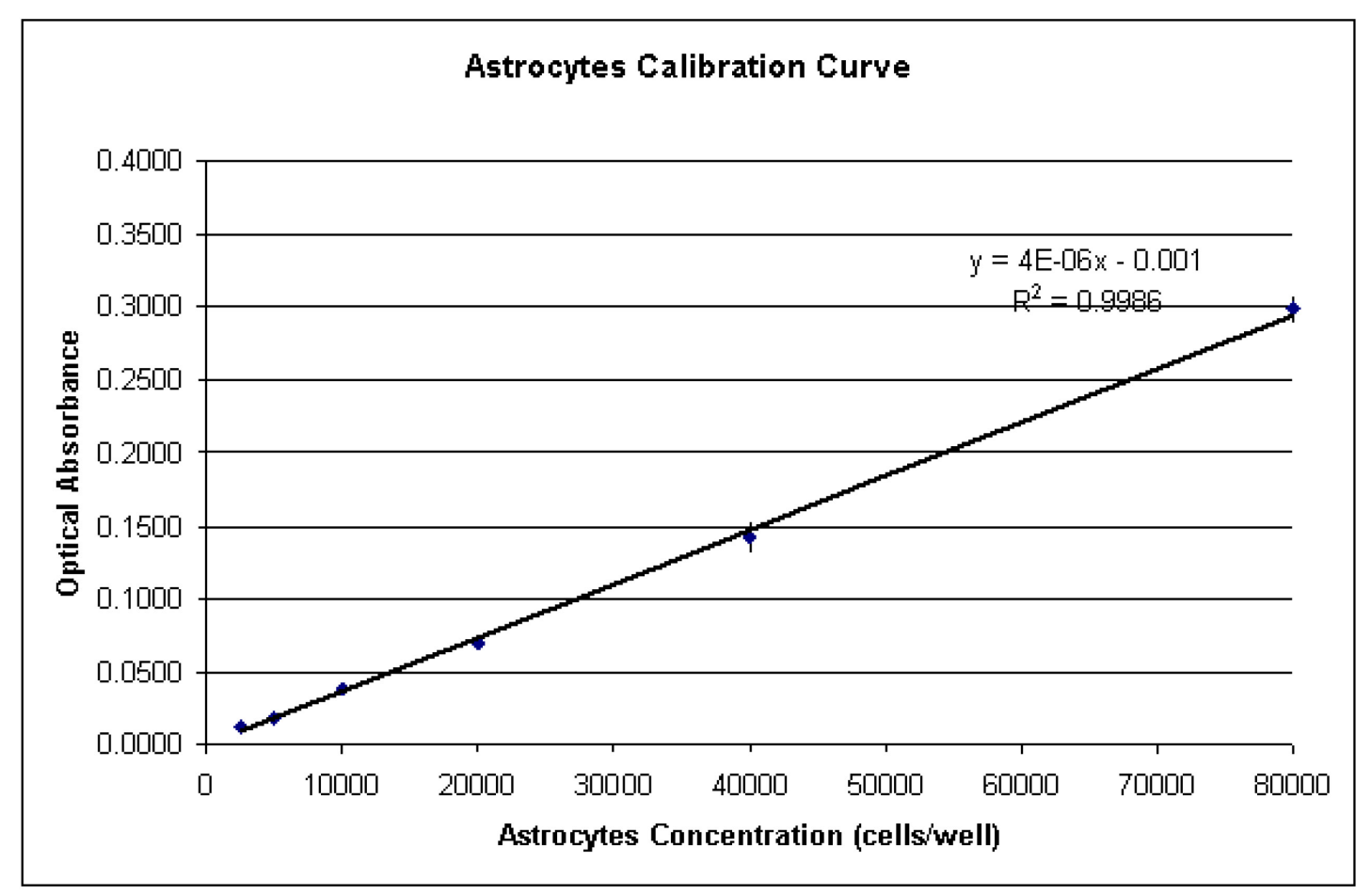

Figure 44 Astrocytes SRB Calibration Curve

Table 7 Cells fell onto well bottoms from different co-culture setups in 4 trials

\begin{tabular}{|c|c|c|c|c|c|}
\hline \multicolumn{2}{|c|}{ Co-culture Samples } & $\begin{array}{l}\text { Trial } \\
\# 7\end{array}$ & $\begin{array}{l}\text { Trial } \\
\# 8\end{array}$ & $\begin{array}{l}\text { Trial } \\
\# 9\end{array}$ & $\begin{array}{l}\text { Trial } \\
\# 10\end{array}$ \\
\hline \multirow[t]{2}{*}{ CO Samples } & $\begin{array}{l}\text { Cells on well bottom } \\
\text { (cells) }\end{array}$ & 75942 & 78856 & 79938 & 76975 \\
\hline & Standard Deviation (cells) & 1460 & 1004 & 801 & 1560 \\
\hline \multirow{2}{*}{$\begin{array}{l}\mathrm{CO}+\mathrm{aECM} \\
\text { Samples }\end{array}$} & $\begin{array}{l}\text { Cells on well bottom } \\
\text { (cells) }\end{array}$ & 37400 & 42069 & 38658 & 42950 \\
\hline & Standard Deviation (cells) & 1172 & 1223 & 1712 & 1272 \\
\hline
\end{tabular}

Thus in summary, the average value of astrocytes fell onto the well bottoms from the 4 trials and their standard deviations are shown in Figure 45. 


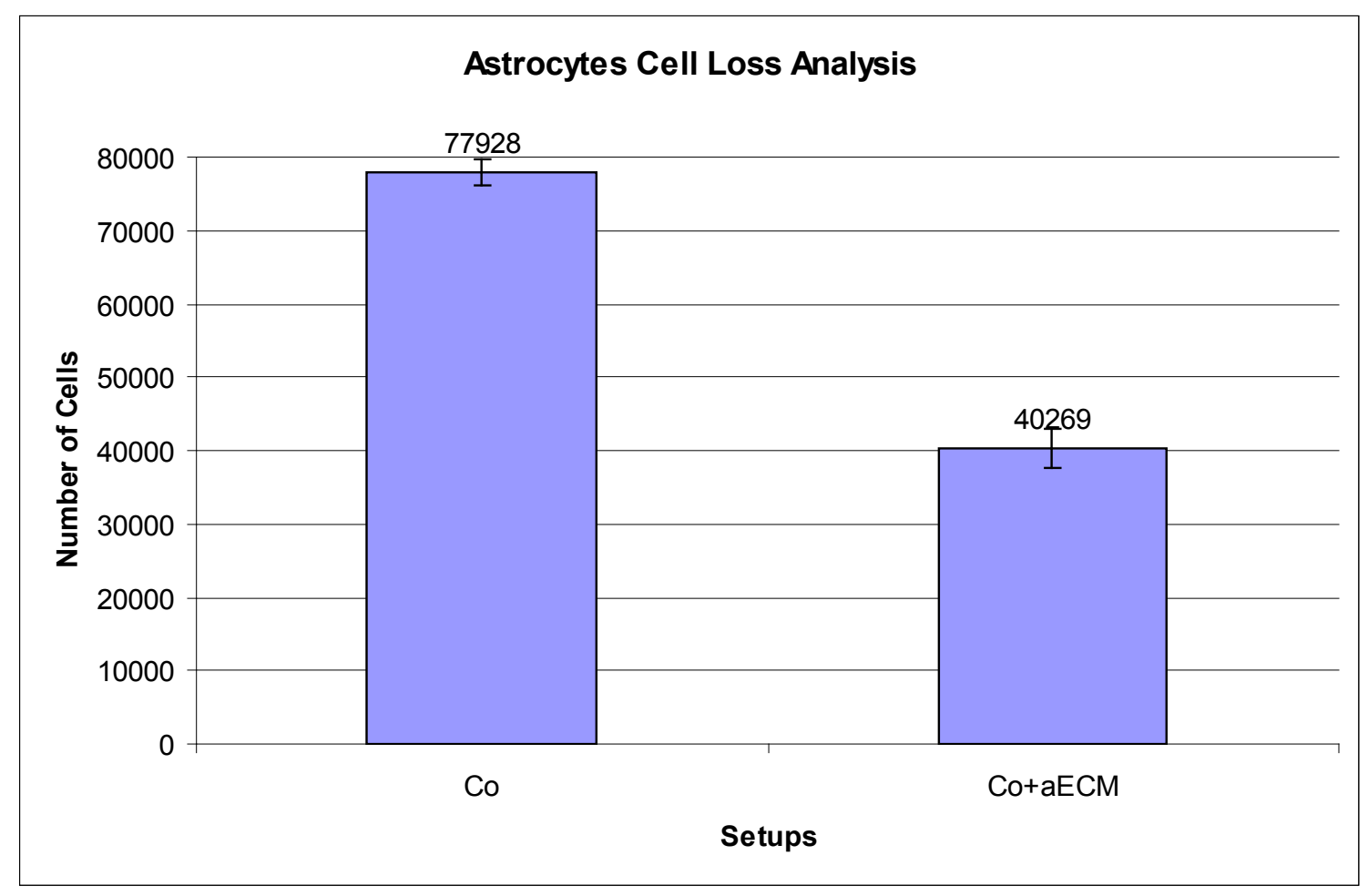

Figure 45 Astrocytes cell loss analysis

\subsection{Discussions and Summary}

As one of the minor study of co-culture model characteristics, the SRB staining study was performed to check the number of astrocytes fell onto well bottoms during the whole culture period. For both co-cultured samples, since the astrocytes were growing on the basal sides of the inserts, the cells would inevitably fall onto the wells because of gravity. However, the SRB assay revealed the fact that the $\mathrm{CO}+\mathrm{aECM}$ samples had much less astrocytes loss from the basal sides of the inserts. Cell loss from the two setups were proven to be statistically significant by t-test analysis when $\alpha=0.05\left(\mathrm{p}=1.98 \times 10^{-7}\right)$. The astrocytes loss results may due to the fact that astrocyte itself is involved with secretion of cellular adhesion molecules, a component of the extracellular matrix. With the existence of aECM, the co-culture present as a better mimic of the real $\mathrm{BBB}$ and the 
extracellular components from the aECM helped the astrocytes on the basal side "stick" tighter to fight the pull from gravity. The integrity of the BBB is also partly revealed in part by this study. On the other hand, the number of cells revealed in this study should not be taken as the actual amount of cells that fell onto the well bottoms. Any cell that fell had the tendency to re-attach to the well bottom and would most likely to reproliferate. The most accurate way should be a day-by-day measurement of the cell number on the well bottoms. But, this would need excessive amount of cell culture apparatus in this minor study of the dissertation and was not considered to be worth doing. Anyway, since cell would grow in all the wells when they fell, we could still use the significantly different numbers in the assessment of astrocyte adhesion to the insert membranes. In the future, analyses of the cellular component of the aECM are recommended to be carried out to better understand the mechanism of this phenomenon. 


\section{CHAPTER X CONCLUSIONS}

The main focus of this specific study can be broken down into two independent but related facets: 1 . to investigate the feasibility of using commercially available immortalized cell lines as an effective alternative of primary cell cultures in BBB in vitro modeling; 2. to explore the effect of astrocyte derived aECM as an endothelial cell seeding scaffold on in vitro BBB co-culture models.

Even though primary cell cultures seem to have higher in vitro - in vivo correlations in terms of trans-endothelial resistance values and expression of tight junction markers, the drawbacks are also quite obvious. First and foremost, the source of cells is limited and unreliable, let alone meeting the requirement of desirable large quantity; second, variations of cellular features are inevitable among different operators; third, primary cell culture can be only maintained for very limited passages before losing their characteristics, apoptosis and altered cytokine susceptibility; last but not least, the demand for primary cells created ethical problems for scientist in using animals (103). On the other hand, immortalized cell lines have their attractive advantages. They are ready to be purchased from reliable commercial sources at relatively low costs in comparison with all the work and effort that have to be put into primary cell culture. Immortalized cell lines get the name because of a procedure in the immortalization where cells are usually transfected by SV-40 virus which enables the cells to virtually escape from normal cell cycle. Immortalized cell lines can usually be cultured for more than 25 passages before any cell crisis, enabling large scale experimental design. The passage numbers of the cells used in the 12 trials of assessment were from passage 2 to passage 10 . No 
significant difference was observed between samples made from different passages of the cells, thus the consistency of the in vitro samples was made possible.

The in vitro " $\mathrm{CO}+\mathrm{aECM"} \mathrm{model} \mathrm{demonstrated} \mathrm{several} \mathrm{important} \mathrm{characteristics}$ in terms of TEER values and occludin expression comparable to literature values such as described by Garcia-Garcia et al. and Hurst et al.. The addition of one extra layer of aECM may be argued to be the reason of higher TEER values and lower mass transport. However it is important to notice the higher tight junction protein occludin expression qualitatively demonstrated by both Western Blot and immuno-fluorescence microscopy. It was also interesting to discover that $\mathrm{CO}+\mathrm{aECM}$ samples had less cell loss on the basal sides of the inserts comparing to $\mathrm{CO}$ samples in the end of the experiments. This phenomenon may indicate the important role astrocytes played in extracellular matrix secretion as well as the interaction between astrocytes and endothelial cells. In the meanwhile, it was experimentally observed that the aECM gradually became less visible throughout the 9-day culture period. This phenomenon can be explained by the extra cellular components secreted by the astrocytes which were gradually consumed by the co-culture system and thus the "extra" layer of aECM was diminishing over time. However, the trends of the quantitative results (TEER and transport) didn't change over time with the disappearing of the aECM. This suggested that the aECM were better mimic of the blood-brain barrier basement membrane and helped the ECs to maintain the tight junction features. Astrocyte derived aECM thus proved its important role in providing a suitable basement membrane environment for the tight junction formation in every aspect. 
A relevant in vitro BBB model should be reproducible, contain cells that are able to retain their functional, morphological and barrier properties. The results of the evaluation of 12 trials of experiments, at least 3 trials contributed to a specific aspect of the samples, all carried out under the same experimental conditions, suggested that the co-cultured sample with aECM serves as a feasible in vitro BBB model, where the aECM provides a biomimick of a basement membrane that is similar to the actual brain microvasculature environment for the endothelial cells. The novel co-cultured BBB in vitro model with aECM was also proved to be reproducible, reliable and easy to manage by using commercially available immortalized cell lines, providing a convenient and cost effective alternative platform for in vitro drug testing and development.

Even though the in vitro model has been demonstrated as a promising relevant BBB model, some works were initially planned but was not able to be accomplished due to the availability of funding and on campus facility. As the future direction of this particular research, possible supplement in the growth media may be explored to further enhance the tight junction formation. More transport studies of more diverse selection of compounds are suggested to be performed on the established in vitro model, so passive transport and active transport of different compounds can be evaluated. And to better understand the in vitro model, characterization of the molecular components in the aECM is suggested. If possible, assay of p-Glycoprotein should also be employed in the further studies. 


\section{LIST OF REFERENCES}

1. Pardridge WM. Why is the global CNS pharmaceutical market so underpenetrated? Drug discovery today. 2002; 7(1): 5-7.

2. Garcia-Garcia E, Gil S, Andrieux K, Desmaele D, Nicolas V, Taran F, et al. A relevant in vitro rat model for the evaluation of blood-brain barrier translocation of nanoparticles. Cellular and Molecular Life Sciences. 2005; 62(12): 1400-1408.

3. Engelhardt B. Development of the blood-brain barrier. Cell and tissue research. 2003; 314(1): 119-129.

4. Hawkins BT, Davis TP. The blood-brain barrier/neurovascular unit in health and disease. Pharmacological reviews. 2005; 57(2): 173-185.

5. Zlokovic BV. The blood-brain barrier in health and chronic neurodegenerative disorders. Neuron. 2008; 57(2): 178-201.

6. Abbott NJ. Prediction of blood-brain barrier permeation in drug discovery from in vivo, in vitro and in silico models. Drug Discovery Today: Technologies. 2004; 1(4): 407-416.

7. Garberg $\mathrm{P}$, Ball $\mathrm{M}$, Borg $\mathrm{N}$, Cecchelli R, Fenart L, Hurst RD, et al. In vitro models for the blood-brain barrier. Toxicology in vitro. 2005; 19(3): 299-334.

8. Wells WA, Bonetta L. Endothelial tight junctions form the blood-brain barrier. J Cell Biol. 2005 May 9; 169(3): 378-379.

9. Ribatti D, Nico B, Crivellato E, Artico M. Development of the blood-brain barrier: A historical point of view. The Anatomical Record Part B: The New Anatomist. 2006; 289(1): 3-8.

10. Friedemann U. Blood-brain barrier. Physiological Reviews. 1942; 22(2): 125-145.

11. Rubin LL, Staddon JM. The cell biology of the blood-brain barrier. Annual review of neuroscience. 1999; 22(1): 11-28.

12. Pardridge WM. Blood-brain barrier drug targeting: the future of brain drug development. Molecular interventions. 2003; 3(2): 90-105.

13. Abbott NJ, Romero IA. Transporting therapeutics across the blood-brain barrier. Molecular Medicine Today. 1996; 2(3): 106-113.

14. Pardridge WM. Brain drug targeting: the future of brain drug development: Cambridge Univ Pr; 2001. 
15. Ajay BGW, Murcko MA. Designing libraries with CNS activity. J Med Chem. 1999;42(24): 4942-4951.

16. Ghose AK, Viswanadhan VN, Wendoloski JJ. A knowledge-based approach in designing combinatorial or medicinal chemistry libraries for drug discovery. 1. A qualitative and quantitative characterization of known drug databases. J Comb Chem. 1999; 1(1): 55-68.

17. Lipinski CA. Drug-like properties and the causes of poor solubility and poor permeability. Journal of pharmacological and toxicological methods. 2000;44(1):235-49.

18. Pardridge WM. Drug delivery to the brain. J Cereb Blood Flow Metab. 1997 Jul; 17(7): 713-731.

19. Abbott NJ, Rönnbäck L, Hansson E. Astrocyte-endothelial interactions at the blood-brain barrier. Nature Reviews Neuroscience. 2006; 7(1): 41-53.

20. Pardridge WM, Golden PL, Kang YS, Bickel U. Brain microvascular and astrocyte localization of P-glycoprotein. Journal of neurochemistry. 1997; 68(3): 12781285.

21. Golden PL, Pardridge WM. P-glycoprotein on astrocyte foot processes of unfixed isolated human brain capillaries. Brain research. 1999; 819(1-2): 143-146.

22. El Hafny B, Chappey O, Piciotti M, Debray M, Boval B, Roux F. Modulation of P-glycoprotein activity by glial factors and retinoic acid in an immortalized rat brain microvessel endothelial cell line. Neuroscience letters. 1997; 236(2): 107-111.

23. Schinkel AH, Wagenaar E, Mol CA, Van Deemter L. P-glycoprotein in the bloodbrain barrier of mice influences the brain penetration and pharmacological activity of many drugs. Journal of Clinical Investigation. 1996; 97(11): 2517-2524.

24. Flaten GE, Dhanikula AB, Luthman K, Brandl M. Drug permeability across a phospholipid vesicle based barrier: A novel approach for studying passive diffusion. European journal of pharmaceutical sciences. 2006; 27(1): 80-90.

25. Pardridge WM. Blood-brain barrier biology and methodology. Journal of neurovirology. 1999; 5(6): 556-569.

26. Haseloff RF, Blasig IE, Bauer HC, Bauer H. In search of the astrocytic factor (s) modulating blood-brain barrier functions in brain capillary endothelial cells in vitro. Cellular and molecular neurobiology. 2005; 25(1): 25-39.

27. Wang DD, Bordey A. The astrocyte odyssey. Progress in neurobiology. 2008; 86(4): 342-367. 
28. Yoo JW, Kim YS, Lee SH, Lee MK, Roh HJ, Jhun BH, et al. Serially passaged human nasal epithelial cell monolayer for in vitro drug transport studies. Pharmaceutical research. 2003; 20(10): 1690-1696.

29. Furuse M, Hirase T, Itoh M, Nagafuchi A, Yonemura S, Tsukita S. Occludin: a novel integral membrane protein localizing at tight junctions. Journal of Cell Biology. 1993; 123(6): 1777-1788.

30. Barrios-Rodiles M, Brown KR, Ozdamar B, Bose R, Liu Z, Donovan RS, et al. High-throughput mapping of a dynamic signaling network in mammalian cells. Science's STKE. 2005; 307(5715): 1621-1625.

31. Li D, Mrsny RJ. Oncogenic Raf-1 disrupts epithelial tight junctions via downregulation of occludin. Journal of Cell Biology. 2000; 148(4): 791-800.

32. Yu ASL, McCarthy KM, Francis SA, McCormack JM, Lai J, Rogers RA, et al. Knockdown of occludin expression leads to diverse phenotypic alterations in epithelial cells. American Journal of Physiology- Cell Physiology. 2005; 288(6): C1231-C1241.

33. Murata M, Kojima T, Yamamoto T, Go M, Takano K, Osanai M, et al. Downregulation of survival signaling through MAPK and Akt in occludin-deficient mouse hepatocytes in vitro. Experimental cell research. 2005; 310(1): 140-151.

34. Osanai M, Murata M, Nishikiori N, Chiba H, Kojima T, Sawada N. Epigenetic silencing of occludin promotes tumorigenic and metastatic properties of cancer cells via modulations of unique sets of apoptosis-associated genes. Cancer research. 2006; 66(18): 9125-9133.

35. Chiba H, Osanai M, Murata M, Kojima T, Sawada N. Transmembrane proteins of tight junctions. Biochimica et Biophysica Acta (BBA)-Biomembranes. 2008; 1778(3): 588-600.

36. Ballabh P, Braun A, Nedergaard M. The blood-brain barrier: an overview: Structure, regulation, and clinical implications. Neurobiology of disease. 2004; 16(1): 113.

37. Davson H, Oldendorf WH. Symposium on membrane transport. Transport in the central nervous system. Proceedings of the Royal Society of Medicine. 1967; 60(4): 326330.

38. Janzer RC, Raff MC. Astrocytes induce blood-brain barrier properties in endothelial cells. Letters to Nature. 1987; 325: 253-257. 
39. Stewart PA, Wiley MJ. Developing nervous tissue induces formation of bloodbrain barrier characteristics in invading endothelial cells: a study using quail-chick transplantation chimeras. Developmental Biology (USA). 1981.

40. Kacem K, Lacombe P, Seylaz J, Bonvento G. Structural organization of the perivascular astrocyte endfeet and their relationship with the endothelial glucose transporter: a confocal microscopy study. Glia. 1998; 23(1): 1-10.

41. Rubin LL, Barbu K, Bard F, Cannon C, Hall DE, Horner H, et al. Differentiation of brain endothelial cells in cell culture. Annals of the New York Academy of Sciences. 2006; 633(Glial-Neuronal Interaction):420-425.

42. Bauer HC, Bauer H. Neural induction of the blood-brain barrier: still an enigma. Cellular and Molecular Neurobiology. 2000; 20(1): 13-28.

43. Dehouck B, Dehouck MP, Fruchart JC, Cecchelli R. Upregulation of the low density lipoprotein receptor at the blood-brain barrier: intercommunications between brain capillary endothelial cells and astrocytes. Journal of Cell Biology. 1994; 126(2): 465-473.

44. Hurst RD, Fritz IB. Properties of an immortalised vascular endothelial/glioma cell co-culture model of the blood-brain barrier. Journal of cellular physiology. 1998; 167(1): 81-88.

45. Lafarga M, Palacios G. Ultrastructural study of pericytes in the rat supraoptic nucleus. Journal of Anatomy. 1975; 120(Pt 3): 433-438.

46. Farrell CR, Stewart PA, Farrell CL, Del Maestro RF. Pericytes in human cerebral microvasculature. The Anatomical Record. 2005; 218(4): 466-469.

47. Rhodin JAG. Ultrastructure of mammalian venous capillaries, venules, and small collecting veins1. Journal of Ultrastructure Research. 1968; 25(5-6): 452-500.

48. Lindahl P, Johansson BR, Leveen P, Betsholtz C. Pericyte loss and microaneurysm formation in PDGF-B-deficient mice. Science. 1997; 277(5323): 242245.

49. Kern TS, Engerman RL. Capillary lesions develop in retina rather than cerebral cortex in diabetes and experimental galactosemia. Archives of ophthalmology. 1996; 114(3): 306-310.

50. Balabanov R, Dore-Duffy P. Role of the CNS microvascular pericyte in the blood-brain barrier. Journal of neuroscience research. 1998; 53(6): 637-644. 
51. Scheithauer BW, Rubinstein LJ, Herman MM. Leukoencephalopathy in Waldenstrom's Macroglobulinemia. Journal of Neuropathology \& Experimental Neurology. 1984; 43(4): 408.

52. Palmer M, Green WR, Maumenee IH, Valle DL, Singer HS, Morton SJ, et al. Niemann-Pick Disease--Type C: Ocular Histopathologic and Electron Microscopic Studies. Archives of Ophthalmology. 1985; 103(6): 817-822.

53. Ramsauer M, Krause D, Dermietzel R. Angiogenesis of the blood-brain barrier in vitro and the function of cerebral pericytes. The FASEB Journal. 2002: 108-141.

54. Bauer HC, Bauer H, Lametschwandtner A, Amberger A, Ruiz P, Steiner M. Neovascularization and the appearance of morphological characteristics of the bloodbrain barrier in the embryonic mouse central nervous system. Developmental Brain Research. 1993; 75(2): 269-278.

55. Kimelberg HK. The problem of astrocyte identity. Neurochemistry international. 2004; 45(2-3):191-202.

56. Liu Y, Rao MS. Glial progenitors in the CNS and possible lineage relationships among them. Biology of the Cell. 2004; 96(4): 279-290.

57. Carmen J, Magnus T, Cassiani-Ingoni R, Sherman L, Rao MS, Mattson MP. Revisiting the astrocyte-oligodendrocyte relationship in the adult CNS. Progress in neurobiology. 2007; 82(3): 151-162.

58. Cai J, Chen Y, Cai WH, Hurlock EC, Wu H, Kernie SG, et al. A crucial role for Olig2 in white matter astrocyte development. Development. 2007; 134(10): 1887-1899.

59. Steinhauser C, Berger T, Frotscher M, Kettenmann H. Heterogeneity in the membrane current pattern of identified glial cells in the hippocampal slice. European Journal of Neuroscience. 2006; 4(6): 472-84.

60. Bordey A, Sontheimer H. Ion channel expression by astrocytes in situ: comparison of different CNS regions. Glia. 2000; 30(1): 27-38.

61. Owens T. Toll-like receptors on astrocytes: patterning for immunity. Journal of Neuroimmunology. 2005; 159(1-2): 1-2.

62. Vaccarino FM, Fagel DM, Ganat Y, Maragnoli ME, Ment LR, Ohkubo Y, et al. Astroglial cells in development, regeneration, and repair. The Neuroscientist. 2007; 13(2): 173-185. 
63. Fernandez-Ruiz J, Romero J, Velasco G, Tolon RM, Ramos JA, Guzman M. Cannabinoid CB2 receptor: a new target for controlling neural cell survival? Trends in pharmacological sciences. 2007; 28(1): 39-45.

64. Furuta A, Wada E, Wada K. Function of glial G-protein coupled receptors. Brain and nerve. 2007; 59(7): 717-724.

65. Lalo U, Pankratov Y, Kirchhoff F, North RA, Verkhratsky A. NMDA receptors mediate neuron-to-glia signaling in mouse cortical astrocytes. Journal of Neuroscience. 2006; 26(10): 2673-2683.

66. Geiger JR, Melcher T, Koh DS, Sakmann B, Seeburg PH, Jonas P, et al. Relative abundance of subunit mRNAs determines gating and $\mathrm{Ca} 2+$ permeability of AMPA receptors in principal neurons and interneurons in rat CNS. Neuron. 1995; 15(1): 193204.

67. Liu B, Neufeld AH. Activation of epidermal growth factor receptors in astrocytes: from development to neural injury. Journal of neuroscience research. 2007; 85(16): 35233529.

68. Milner R, Campbell IL. Developmental Regulation of [beta] 1 Integrins during Angiogenesis in the Central Nervous System. Molecular and Cellular Neuroscience. 2002; 20(4): 616-626.

69. Haring HP, Akamine P, Habermann R, Koziol JA, Del Zoppo GJ. Distribution of integrin-like immunoreactivity on primate brain microvasculature. Journal of Neuropathology \& Experimental Neurology. 1996; 55(2): 236.

70. Rist RJ, Romero IA, Chan MWK, Couraud PO, Roux F, Abbott NJ. F-actin cytoskeleton and sucrose permeability of immortalised rat brain microvascular endothelial cell monolayers: effects of cyclic AMP and astrocytic factors. Brain research. 1997; 768(1-2): 10-18.

71. Sobue K, Yamamoto N, Yoneda K, Hodgson ME, Yamashiro K, Tsuruoka N, et al. Induction of blood-brain barrier properties in immortalized bovine brain endothelial cells by astrocytic factors. Neuroscience research. 1999; 35(2): 155-164.

72. Reichel A, Begley DJ, Abbott NJ. An overview of in vitro techniques for bloodbrain barrier studies. Methods in Molecular Medicine. 2003; 89: 307-24.

73. Estrada C, Bready JV, Berliner JA, Pardridge WM, Cancilla PA. Astrocyte Growth Stimulation by a Soluble Factor Produced by Cerebral Endothelial Cellsin vitro. Journal of Neuropathology \& Experimental Neurology. 1990; 49(6): 539. 
74. Shea TB, Beermann ML, Nixon RA. Sequential effects of astroglial-derived factors on neurite outgrowth: Initiation by protease inhibitors and potentiation by extracellular matrix components. Journal of neuroscience research. 2004; 31(2): 309-317.

75. Smith GM, Rutishauser U, Silver J, Miller RH. Maturation of astrocytes in vitro alters the extent and molecular basis of neurite outgrowth* 1 . Developmental biology. 1990; 138(2): 377-390.

76. Matthiessen HP, Schmalenbach C, Muler HW. Astroglia-released neurite growthinducing activity for embryonic hippocampal neurons is associated with laminin bound in a sulfated complex and free fibronectin. Glia. 2004; 2(3): 177-188.

77. Yin KJ, Cirrito JR, Yan P, Hu X, Xiao Q, Pan X, et al. Matrix metalloproteinases expressed by astrocytes mediate extracellular amyloid-beta peptide catabolism. Journal of Neuroscience. 2006; 26(43): 10939-10948.

78. Yong W. Matrix metalloproteinases and diseases of the CNS. Trends in neurosciences. 1998; 21(2): 75-80.

79. Muir EM, Adcock KH, Morgenstern DA, Clayton R, Von Stillfried N, Rhodes K, et al. Matrix metalloproteases and their inhibitors are produced by overlapping populations of activated astrocytes. Molecular Brain Research. 2002; 100(1-2): 103-117.

80. Ojeda SR, Prevot V, Heger S, Lomniczi A, Dziedzic B, Mungenast A. Glia-toneuron signaling and the neuroendocrine control of female puberty. Annals of medicine. 2003; 35(4): 244-255.

81. Schaeffer WI. Proposed usage of animal tissue culture terms (Revised 1978) Usage of vertebrate cell, tissue and organ culture terminology. In Vitro Cellular \& Developmental Biology-Plant. 1979; 15(9):649-653.

82. Diglio CA, Grammas P, Giacomelli F, Wiener J. Primary culture of rat cerebral microvascular endothelial cells. Isolation, growth, and characterization. Laboratory investigation; a journal of technical methods and pathology. 1982; 46(6): 554.

83. Pardridge WM. Blood-brain barrier methodology and biology. Introduction to the blood-brain barrier: methodology, biology, and pathology. 1998: 1.

84. Gray TE, Guzman K, Davis CW, Abdullah LH, Nettesheim P. Mucociliary differentiation of serially passaged normal human tracheobronchial epithelial cells. American journal of respiratory cell and molecular biology. 1996; 14(1): 104-112.

85. Levashova ZB, Plisov SY, Perantoni AO. Conditionally immortalized cell line of inducible metanephric mesenchyme. Kidney international. 2003; 63(6): 2075-2087. 
86. Veronesi B. Characterization of the MDCK cell line for screening neurotoxicants. Neurotoxicology. 1996; 17(2): 433-443.

87. Hurst RD, Heales SJR, Dobbie MS, Barker JE, Clark JB. Decreased endothelial cell glutathione and increased sensitivity to oxidative stress in an in vitro blood-brain barrier model system. Brain research. 1998; 802(1-2): 232-240.

88. Kuchler-Bopp S, Delaunoy JP, Artault JC, Zaepfel M, Dietrich JB. Astrocytes induce several blood-brain barrier properties in non-neural endothelial cells. Neuroreport. 1999; 10(6): 1347-1353.

89. Artursson P, Karlsson J, Ocklind G, Schipper N, Karlsson J. Studying transport processes in absorptive epithelia. Epithelial Cell Culture: A Practical Approach. 1996: $111-129$.

90. Yee S. In vitro permeability across Caco-2 cells (colonic) can predict in vivo (small intestinal) absorption in man - fact or myth. Pharmaceutical research. 1997; 14(6): 763-766.

91. Artursson P, Palm K, Luthman K. Caco-2 monolayers in experimental and theoretical predictions of drug transport. Advanced drug delivery reviews. 1996; 22(1-2): $67-84$.

92. Lundquist S, Renftel M, Brillault J, Fenart L, Cecchelli R, Dehouck MP. Prediction of drug transport through the blood-brain barrier in vivo: a comparison between two in vitro cell models. Pharmaceutical research. 2002; 19(7): 976-981.

93. Rubin LL, Hall DE, Porter S, Barbu K, Cannon C, Horner HC, et al. A cell culture model of the blood-brain barrier. Journal of Cell Biology. 1991; 115(6): 17251735.

94. Deli MA, Dehouck MP, Abraham CS, Cecchelli R, Joo F. Penetration of small molecular weight substances through cultured bovine brain capillary endothelial cell monolayers: the early effects of cyclic adenosine 3', 5'-monophosphate. Experimental physiology. 1995; 80(4): 675-678.

95. Raub TJ. Signal transduction and glial cell modulation of cultured brain microvessel endothelial cell tight junctions. American Journal of Physiology- Cell Physiology. 1996; 271(2): C495-C503.

96. Behonick DJ, Werb Z. A bit of give and take: the relationship between the extracellular matrix and the developing chondrocyte. Mechanisms of development. 2003; 120(11): 1327-1336. 
97. Mizuguchi H, Utoguchi N, Mayumi T. Preparation of glial extracellular matrix: a novel method to analyze glial-endothelial cell interaction. Brain Research Protocols. 1997; 1(4): 339-343.

98. Radany EH, Brenner M, Besnard F, Bigornia V, Bishop JM, Deschepper CF. Directed establishment of rat brain cell lines with the phenotypic characteristics of type 1 astrocytes. Proceedings of the National Academy of Sciences. 1992; 89(14): 6467-6471.

99. McCall AL. Cerebral glucose metabolism in diabetes mellitus. European journal of pharmacology. 2004; 490(1-3): 147-158.

100. Nakazono T, Murakami T, Sakai S, Higashi Y, Yata N. Application of microdialysis for study of caffeine distribution into brain and cerebrospinal fluid in rats. Chemical \& pharmaceutical bulletin. 1992; 40(9): 2510.

101. Sadzuka Y, Hatakeyama H, Daimon T, Sonobe T. Screening of biochemical modulator by tumor cell permeability of doxorubicin. International journal of pharmaceutics. 2008; 354(1-2): 63-69.

102. Vichai V, Kirtikara K. Sulforhodamine B colorimetric assay for cytotoxicity screening. Nature protocols. 2006; 1(3): 1112-1116.

103. Herrmann J, Gressner AM, Weiskirchen R. Immortal hepatic stellate cell lines: useful tools to study hepatic stellate cell biology and function? Journal of cellular and molecular medicine. 2007; 11(4): 704-722. 
ZHIQI ZHANG

Nov 5, 1978

$1997-2001$

$2004-2010$

$2005-2008$

$2001-2004$
Born, Daqing, Heilongjiang, China

B.E., Biomedical Engineering

Shanghai Jiao Tong University

Shanghai, China

Applications Engineer

National Instruments

Shanghai, China

Doctoral Candidate in Biomedical Engineering

Florida International University

Miami, FL

Lab Manager

Tissue \& Bioengineering Laboratory

Florida International University

Miami, FL

\section{PUBLICATIONS AND PRESENTATIONS}

Zhiqi. Zhang, Anthony McGoron, Eric Crumpler and Chenzhong Li, "Co-Culture Based Blood-Brain Barrier in Vitro Model, a Tissue Engineering Approach Using Immortalized Cell Lines for Drug Transport Study", Applied Biochemistry and Biotechnology. Accepted.

Zhiqi Zhang, Anthony Conway, Ann Salamone, Eric Crumpler, Xueji Zhang and Chenzhong Li, "Amphiphilic Copolymers for Liquid Bandage Application Studies", Frontiers in Bioscience, 2010, E2, 1123-1133.

Zhiqi Zhang, Eric Crumpler and Chenzhong Li "Blood-Brain Barrier In Vitro Model Utilizing Immortalized Cell Lines" IFMBE Proceedings, 2009, 251-253.

Zhiqi Zhang and Eric Crumpler, "Blood-Brain Barrier in vitro Model: a Tissue Engineering Approach", Biomedical Engineering Recent Developments, Homer Nazeran, Michael Goldman, Richard Schoephoerster, (C) 2008 Medical and Engineering Publishers, Inc. ISBN 978-1-930636-06-4. 
Zhiqi Zhang and Eric T. Crumpler, "A Co-Culture System with aECM for Blood-Brain Barrier In Vitro Model", Presented in Scientific Conference of The Society for Physical Regulation in Biology and Medicine (SPRBM), Miami, FL USA, January 9-11, 2008.

Sa Li, Yonggang Zhao, Zhiqi Zhang and Yuhong Xu, "Liposomal Delivery Vehicles of Paclitaxel", 23rd Annual International Conference of the IEEE Engineering in Medicine and Biology Society, Istanbul, Turkey, October 25-28, 2001. 